\title{
Synthesis of Functionalized Nitroarylmagnesium
}

\section{Halides via an Iodine-Magnesium Exchange}

\author{
Ioannis Sapountzis, Henry Dube, Robert Lewis, Nina Gommermann and Paul Knochel*
}

Department Chemie, Ludwig-Maximilians-University, Butenandtstrasse 5-13, 81377 Munich, Germany, Fax.: +49 (0)89 2180 77680, Paul.Knochel@cup.uni-muenchen.de

\section{Supporting Information}

\section{Table of Contents:}

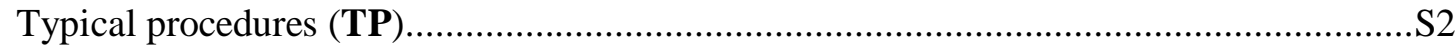

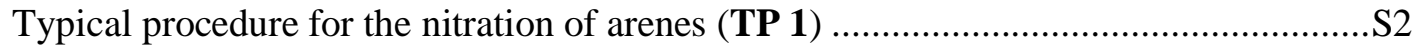

Typical procedure for the iodination of arenes using silver sulfate (TP 2) ....................S2

Typical procedure for the performance of an iodine-magnesium exchange reaction on functionalised $o$-iodonitroarenes (TP 3)

Typical procedure for the preparation of nitro-substituted polyfunctional biphenyls by

Negishi cross-coupling of $o$-nitroarylzinc reagents (TP 4)

Typical procedure for the performance of an iodine-magnesium exchange reaction on

functionalised $m$ - and $p$-iodonitroarenes (TP 5)

Typical procedure for the preparation of nitro-substituted polyfunctional biphenyls by

Negishi cross-coupling of $m$ - and $p$-nitroarylzinc reagents (TP 6)

Typical procedure for the formation of alkyl- and aryl-sulfonates from the corresponding

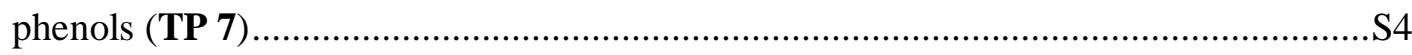

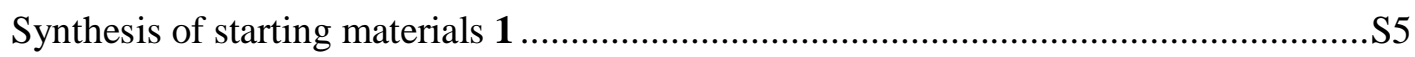

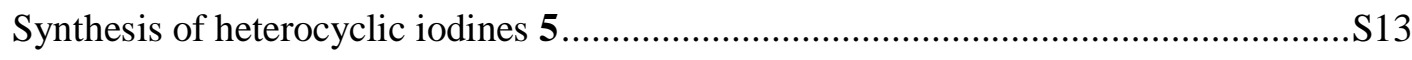

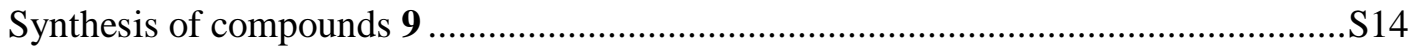

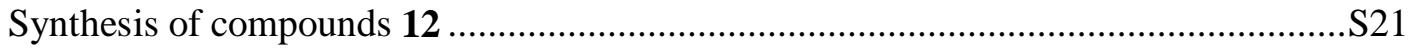

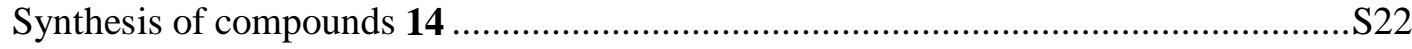

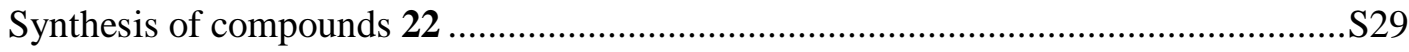


Synthesis of compounds 26 S37

Synthesis of compounds 27 . S39

Synthesis of compounds 29 S45

General All reactions were carried out under an argon atmosphere in oven dried glassware. Elemantal analyses were carried out on a Heraeus CHN-Rapid-Elementanalyzer. THF was continuously refluxed and freshly distilled from sodium benzophenone ketyl under nitrogen. Preparative flash chromatography was performed on silicagel $60(0.040-0.063 \mathrm{~mm})$ from Merck. Yields refer to isolated yields of compounds estimated to be $>95 \%$ pure as determined by ${ }^{1} \mathrm{H}-\mathrm{NMR}$, capillary GC and combustion analysis (new compounds).

\section{Typical procedures (TP)}

\section{Typical procedure for the nitration of arenes (TP 1)}

A cold mixture of $1.9 \mathrm{~mL}$ nitric acid $\left(\mathrm{HNO}_{3}, 100 \%\right)$ and $2.7 \mathrm{~mL}$ sulfuric acid $\left(\mathrm{H}_{2} \mathrm{SO}_{4}, 100 \%\right)$ was added slowly to a solution of the corresponding arene $(10 \mathrm{mmol})$ dissolved in $5 \mathrm{~mL}$ sulphuric acid $\left(\mathrm{H}_{2} \mathrm{SO}_{4}, 100 \%\right)$ within 30 min with vigorous stirring. Upon completion of the addition the solution was allowed to warm to ambient temperature and stirring was continued for $5 \mathrm{~h}$ and the reaction mixture was poured onto crushed ice $(100 \mathrm{~g})$. The solid was filtrated, taken up in ethyl acetate $(100 \mathrm{~mL})$ and washed with saturated $\mathrm{NaHCO}_{3 \text { (aq.) }}(100 \mathrm{~mL})$, dried over $\mathrm{Na}_{2} \mathrm{SO}_{4}$, filtered and concentrated in vacuo. Recrystallization from ethanol yielded the pure product.

\section{Typical procedure for the iodination of arenes using silver sulfate (TP 2) ${ }^{1}$}

A $250 \mathrm{~mL}$ round-bottom flask, equipped with a magnetic stirring bar, was charged with iodine $(5.08 \mathrm{~g}, 20.0 \mathrm{mmol})$ and silver sulfate $(6.23 \mathrm{~g}, 20.0 \mathrm{mmol})$ in ethanol $(100 \mathrm{~mL})$. The aromatic compound $(20 \mathrm{mmol})$ was then added and the mixture was stirred vigorously at $\mathrm{rt}$ until tlc analysis indicated complete conversion (typically $30 \mathrm{~min}-2 \mathrm{~h}$ ). The reaction mixture was filtered through a glass sinter, the solids on the sinter were washed with ethyl acetate $(2 \mathrm{x}$ $100 \mathrm{~mL}$ ) and the filtrate was concentrated in vacuo. The residue was taken up in $\mathrm{CH}_{2} \mathrm{Cl}_{2}$ $(100 \mathrm{~mL})$, washed with $2 \mathrm{M} \mathrm{NaOH}(100 \mathrm{~mL})$, water $(100 \mathrm{~mL})$, dried over $\mathrm{Na}_{2} \mathrm{SO}_{4}$ and concentrated in vacuo. Flash chromatography on silica gel furnished the product.

\section{Typical procedure for the performance of an iodine-magnesium exchange reaction on functionalised $o$-iodonitroarenes (TP 3)}

A dry and argon flushed $25 \mathrm{~mL}$ flask, equipped with a magnetic stirrer and a septum, was charged with the $o$-iodo-nitrobenzene $(2 \mathrm{mmol})$. Dry THF $(5 \mathrm{~mL})$ was added, the mixture cooled to $-40{ }^{\circ} \mathrm{C}$ and $\mathrm{PhMgCl}(2.2 \mathrm{mmol}$, solution in THF) was then added dropwise. The $\mathrm{I} / \mathrm{Mg}$ exchange was usually complete after 5 min (checked by GC analysis of reaction aliquots) and, if necessary, $\mathrm{CuCN} \cdot 2 \mathrm{LiCl}(2 \mathrm{~mL}, 1.0 \mathrm{M}$ in THF) was added prior to the addition of the electrophile. The reaction mixture was stirred for $30 \mathrm{~min}$ at $-40{ }^{\circ} \mathrm{C}$, the cooling bath was removed and the mixture stirred for additional $30 \mathrm{~min}$ at room temperature. The reaction 
mixture was quenched with saturated $\mathrm{NH}_{4} \mathrm{Cl}_{(\mathrm{aq})}(2 \mathrm{~mL})$ and poured into water $(25 \mathrm{~mL})$. The aqueous phase was extracted with ethyl acetate $(2 \times 40 \mathrm{~mL})$, the collected organic phases were washed with brine $(30 \mathrm{~mL})$, dried over $\mathrm{Na}_{2} \mathrm{SO}_{4}$ and concentrated in vacuo. Purification by flash chromatography furnished the product.

\section{Typical procedure for the preparation of nitro-substituted polyfunctional biphenyls by Negishi cross-coupling of $o$-nitroarylzinc reagents (TP 4)}

A dry and argon flushed $25 \mathrm{~mL}$ flask, equipped with a magnetic stirrer and a septum, was charged with the 2-iodonitroarene $(1.00 \mathrm{mmol})$. Dry THF $(5 \mathrm{~mL})$ was added, the mixture cooled to $-40{ }^{\circ} \mathrm{C}$ and mesitylmagnesium bromide $(1.6 \mathrm{~mL}, 1.1 \mathrm{mmol}, 0.70 \mathrm{M}$ in THF) was added dropwise. The I/Mg exchange was complete after 15 min (checked by GC analysis of reaction aliquots) and zinc bromide (1.1 mL, $1.1 \mathrm{mmol}, 1.0 \mathrm{M}$ in THF) was added to the magnesiated nitroarene. Another dry two-necked flask equipped with a magnetic stirrer and a septum was charged with bis-(dibenzylideneacetone)palladium(0) $\left(\operatorname{Pd}(\mathrm{dba})_{2}\right) \quad(29 \mathrm{mg}$, $5 \mathrm{~mol} \%)$ and tris-o-furylphosphine (tfp) $(25 \mathrm{mg}, 10 \mathrm{~mol} \%)$ followed by THF (2 mL). The initial red colour disappeared after 2 min leading to a yellow solution and haloarene $(1.5 \mathrm{mmol})$ was added. This solution was added via cannula after $10 \mathrm{~min}$ of stirring to the reaction mixture at $-40{ }^{\circ} \mathrm{C}$ and the cooling bath was removed. The reaction was stirred for $5 \mathrm{~h}$ at room temperature, treated with ethanol $(2 \mathrm{~mL})$ and poured into water $(25 \mathrm{~mL})$. The aqueous phase was extracted with ethyl acetate $(3 \times 40 \mathrm{~mL})$, the collected organic phases were washed with brine $(30 \mathrm{~mL})$, dried over $\mathrm{Na}_{2} \mathrm{SO}_{4}$ and concentrated in vacuo. Purification by flash chromatography furnished the product.

\section{Typical procedure for the performance of an iodine-magnesium exchange reaction on functionalised $\boldsymbol{m}$ - and $\boldsymbol{p}$-iodonitroarenes (TP 5)}

A dry and argon flushed $25 \mathrm{~mL}$ flask, equipped with a magnetic stirrer and a septum, was charged with the $m$ - or $p$-iodo-nitrobenzene $(1 \mathrm{mmol})$. Dry THF $(3 \mathrm{~mL})$ was added, the mixture cooled to $-78{ }^{\circ} \mathrm{C}$ and $\mathrm{PhMgCl}(1.1 \mathrm{mmol}$, solution in THF) was then added dropwise. The I/Mg exchange was usually complete after 30 min (checked by GC analysis of reaction aliquots) and, if necessary, $\mathrm{CuCN} \cdot 2 \mathrm{LiCl}(2 \mathrm{~mL}, 1.0 \mathrm{M}$ in THF) was added prior to the addition of the electrophile. The reaction mixture was stirred for $30 \mathrm{~min}$ at $-78{ }^{\circ} \mathrm{C}$, the cooling bath was removed and the reaction mixture stirred for additional $30 \mathrm{~min}$ at ambient temperature. The reaction mixture was quenched with saturated $\mathrm{NH}_{4} \mathrm{Cl}_{(\mathrm{aq})}(2 \mathrm{~mL})$ and poured into water $(25 \mathrm{~mL})$. The aqueous phase was extracted with ethyl acetate $(2 \times 40 \mathrm{~mL})$, the collected organic phases were washed with brine $(30 \mathrm{~mL})$, dried over $\mathrm{Na}_{2} \mathrm{SO}_{4}$, filtered and concentrated in vacuo. Purification by flash chromatography furnished the product.

\section{Typical procedure for the preparation of nitro-substituted polyfunctional biphenyls by Negishi cross-coupling of $m$ - and $p$-nitroarylzinc reagents (TP 6)}

A dry and argon flushed $25 \mathrm{~mL}$ flask, equipped with a magnetic stirrer and a septum, was charged with $m$ - or $p$-iodonitrobenzene $(1.0 \mathrm{mmol})$. Dry THF $(5 \mathrm{~mL})$ was added, the mixture cooled to $-78{ }^{\circ} \mathrm{C}$ and $\mathrm{PhMgCl}(0.65 \mathrm{~mL}, 1.05 \mathrm{mmol}$, solution in THF) was added dropwise. The I/Mg exchange was complete after $10 \mathrm{~min}$ (checked by GC analysis of reaction aliquots) and zinc bromide (1.1 mL, $1.1 \mathrm{mmol}, 1.0 \mathrm{M}$ in THF) was added to the magnesiated benzoate 
at $-78{ }^{\circ} \mathrm{C}$. Another dry two-necked flask equipped with a magnetic stirrer and a septum was charged with bis-(dibenzylideneacetone)palladium(0) $\left(\mathrm{Pd}(\mathrm{dba})_{2}\right)(29 \mathrm{mg}, 5 \mathrm{~mol} \%)$ and tris-ofurylphosphine (tfp) $(25 \mathrm{mg}, 10 \mathrm{~mol} \%)$ followed by THF $(2 \mathrm{~mL})$. The initial red colour disappeared after $2 \mathrm{~min}$ leading to a yellow solution and the iodobenzene $(2 \mathrm{mmol})$ was added. This solution was added via cannula after $10 \mathrm{~min}$ of stirring to the reaction mixture at $-78^{\circ} \mathrm{C}$ and the cooling bath was removed. The reaction mixture was stirred for $2 \mathrm{~h}$ at room temperature, treated with ethanol $(2 \mathrm{~mL})$ and poured into water $(25 \mathrm{~mL})$. The aqueous phase was extracted with ethyl acetate $(3 \times 40 \mathrm{~mL})$, the collected organic phases were washed with brine $(30 \mathrm{~mL})$, dried over $\mathrm{Na}_{2} \mathrm{SO}_{4}$ and concentrated in vacuo. Purification by flash chromatography furnished the product.

\section{Typical procedure for the formation of alkyl- and aryl-sulfonates from the corresponding phenols (TP 7)}

A $100 \mathrm{~mL}$ round-bottom flask, equipped with a magnetic stirring bar, was charged with the corresponding phenol $(10.0 \mathrm{mmol})$ and dry pyridine $(20 \mathrm{~mL})$ was added. The solution was cooled to $0{ }^{\circ} \mathrm{C}$ and the aryl or alkyl sulfonyl chloride $(12.0 \mathrm{mmol})$ was added portionwise. On completion of the addition, the reaction mixture was stirred for additional $15 \mathrm{~min}$ at $0{ }^{\circ} \mathrm{C}$ and was then allowed to warm to room temperature and was stirred overnight. The reaction mixture was poured onto ice $(50 \mathrm{~g})$ and ethyl acetate $(100 \mathrm{~mL}), 2 \mathrm{M} \mathrm{HCl}(30 \mathrm{~mL})$ was added and the phases were separated. The organic phase was washed with $2 \mathrm{M} \mathrm{HCl}(30 \mathrm{~mL})$, saturated $\mathrm{NaHCO}_{3(\mathrm{aq})}(50 \mathrm{~mL})$, brine $(50 \mathrm{~mL})$, dried over $\mathrm{Na}_{2} \mathrm{SO}_{4}$, filtered and concentrated in vacuo. Flash chromatography on silica gel furnished the product. 


\section{Synthesis of starting materials 1}

\section{Synthesis of ethyl 4-iodo-3-nitrobenzoate (1f $)^{2}$}<smiles>CCOC(=O)c1ccc(I)c([N+](=O)[O-])c1</smiles>

Prepared according to TP 1 from $\mathrm{HNO}_{3}(100 \%, 1.9 \mathrm{~mL}), \mathrm{H}_{2} \mathrm{SO}_{4}(100 \%, 2.7 \mathrm{~mL})$ and ethyl 4iodobenzoate $(5.52 \mathrm{~g}, 20.0 \mathrm{mmol})$. Recrystallization from ethanol gave title compound $\mathbf{1 f}$ as yellow solid (5.48 g, 86\%).

mp.: $\quad 89-90{ }^{\circ} \mathrm{C} \quad$ (Lit.: $\left.89.5^{\circ} \mathrm{C}\right) . \quad{ }^{1} \mathbf{H}-\mathbf{N M R} \quad\left(\mathrm{CDCl}_{3}, \quad 300 \mathrm{MHz}, \quad 25^{\circ} \mathrm{C}\right): \quad \delta=8.36 \quad(\mathrm{~d}$, $\left.{ }^{4} J(\mathrm{H}, \mathrm{H})=2.2 \mathrm{~Hz}, 1 \mathrm{H}\right), 8.06 \quad\left(\mathrm{~d},{ }^{3} J(\mathrm{H}, \mathrm{H})=8.9 \mathrm{~Hz} \quad 1 \mathrm{H}\right), \quad 7.80 \quad\left(\mathrm{dd},{ }^{3} J(\mathrm{H}, \mathrm{H})=8.9 \mathrm{~Hz}\right.$, $\left.{ }^{4} J(\mathrm{H}, \mathrm{H})=2.2 \mathrm{~Hz}, 1 \mathrm{H}\right), 4.35\left(\mathrm{q},{ }^{3} J(\mathrm{H}, \mathrm{H})=7.1 \mathrm{~Hz}, 2 \mathrm{H}\right), 1.34\left(\mathrm{t},{ }^{3} J(\mathrm{H}, \mathrm{H})=7.1 \mathrm{~Hz}, 3 \mathrm{H}\right) .{ }^{13} \mathrm{C}-$ NMR $\left(\mathrm{CDCl}_{3}, 75 \mathrm{MHz}, 25^{\circ} \mathrm{C}\right): \delta=164.0,153.2,142.3,133.4,131.9,125.9,91.9,62.1,14.2$. MS (70 eV, EI), m/z (\%): 321 (88) [M+1, 293 (100), 276 (99), 260 (12), 247 (26), 230 (37), 219 (4), 201 (12), 191 (11), 176 (2), 149 (1), 136 (8), 119 (5), 103 (34), 92 (13), 75 (40), 63 (5), 53 (2). IR (KBr): $\tilde{v}=2982$ (m), 1708 (vs), 1600 (s), 1561 (m), 1532 (vs), 1469 (s), 1385 (m), 1366 (m), 1341 (vs), 1285 (vs), 1238 (vs), 1158 (s), 1136 (m), 1116 (m), 1018 (s), 916 (w), 864 (w), 854 (m), 834 (m), 768 (m), 743 (s), 727 (m), 658 (w). $\mathbf{C}_{\mathbf{9}} \mathbf{H}_{\mathbf{8}} \mathbf{I N O}_{\mathbf{4}}$ : required: C: 33.67; H: 2.51; N: 4.36; found: C: 33.76; H: 2.35; N: 4.33 .

\section{Synthesis of 4-iodo-3-nitrobenzonitrile (1g)}

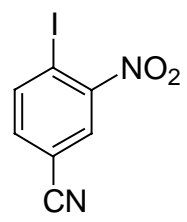

Prepared according to TP 1 from $\mathrm{HNO}_{3}(100 \%, 0.96 \mathrm{~mL}), \mathrm{H}_{2} \mathrm{SO}_{4}(100 \%, 1.4 \mathrm{~mL})$ and 4iodobenzonitrile $(2.30 \mathrm{~g}, 10.0 \mathrm{mmol})$. Recrystallization from ethanol gave title compound $\mathbf{1 g}$ as yellow solid $(1.84 \mathrm{~g}, 67 \%)$.

mp.: $\quad 132-133{ }^{\circ} \mathrm{C} .{ }^{1} \mathbf{H}-\mathbf{N M R}\left(\mathrm{CDCl}_{3}, 300 \mathrm{MHz}, 25^{\circ} \mathrm{C}\right): \delta=8.16\left(\mathrm{~d},{ }^{3} \mathrm{~J}(\mathrm{H}, \mathrm{H})=8.4 \mathrm{~Hz}, 1 \mathrm{H}\right)$, $8.05\left(\mathrm{~d},{ }^{4} J(\mathrm{H}, \mathrm{H})=1.8 \mathrm{~Hz}, 1 \mathrm{H}\right), 7.45\left(\mathrm{dd},{ }^{3} J(\mathrm{H}, \mathrm{H})=8.4 \mathrm{~Hz},{ }^{4} J(\mathrm{H}, \mathrm{H})=1.8 \mathrm{~Hz}, 1 \mathrm{H}\right) .{ }^{13} \mathrm{C}-$ NMR $\left(\mathrm{CDCl}_{3}, 75 \mathrm{MHz}, 25^{\circ} \mathrm{C}\right): \delta=151.0,143.7,135.8,128.8,116.4,114.0,93.1 . \mathrm{MS}$ (70 eV, EI), m/z (\%): 274 (100) [M+], 258 (2), 228 (50), 216 (6), 127 (5), 117 (12), 101 (31), 75 (7), 50 (10). IR (KBr): $\tilde{v}=3091$ (w), 3066 (w), 2236 (s), 1597 (m), 1548 (s), 1530 (vs), 1464 (m), 1352 (s), 1264 (w), 1024 (w), 843 (w), 788 (w), 671 (w), 55 (w). HRMS for $\mathbf{C}_{7} \mathbf{H}_{3} \mathbf{I N}_{2} \mathbf{O}_{2}$ (273.9239): found: 273.9245 . 


\section{Synthesis of pyperidino-4-iodo-3-nitro-benzamide (1h)}

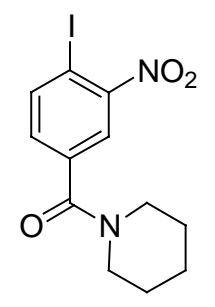

Prepared according to TP 1 from $\mathrm{HNO}_{3}(100 \%, 1.9 \mathrm{~mL}), \mathrm{H}_{2} \mathrm{SO}_{4}(100 \%, 2.7 \mathrm{~mL})$ pyperidino4-iodo-benzamide $(4.73 \mathrm{~g}, 15.0 \mathrm{mmol})$. Recrystallization from ethanol furnished title compound $\mathbf{1 h}$ as colourless solid $(4.50 \mathrm{~g}, 83 \%)$.

mp.: $\quad 155.5-156.5{ }^{\circ} \mathrm{C} .{ }^{1} \mathrm{H}-\mathrm{NMR}\left(400 \mathrm{MHz}, \mathrm{CDCl}_{3}, 25^{\circ} \mathrm{C}\right): \delta=8.06\left(\mathrm{~d},{ }^{3} J(\mathrm{H}, \mathrm{H})=8.0 \mathrm{~Hz}\right.$, $1 \mathrm{H}), 7.86\left(\mathrm{~d},{ }^{4} J(\mathrm{H}, \mathrm{H})=1.9 \mathrm{~Hz}, 1 \mathrm{H}\right), 7.27\left(\mathrm{dd},{ }^{3} J(\mathrm{H}, \mathrm{H})=8.0 \mathrm{~Hz},{ }^{4} J(\mathrm{H}, \mathrm{H})=1.9 \mathrm{~Hz}, 1 \mathrm{H}\right)$, 3.67 (s_br, $2 \mathrm{H}$ ), 3.31 (s_br, $2 \mathrm{H}), 1.72-1.42$ (m, $6 \mathrm{H}) .{ }^{13} \mathbf{C}-\mathbf{N M R}\left(100 \mathrm{MHz}, \mathrm{CDCl}_{3}, 25{ }^{\circ} \mathrm{C}\right.$ ): $\delta=166.7,152.8,142.1,137.7,131.5,123.9,87.2,48.8,43.4,26.5,25.4,24.3 . \mathbf{M S}(70 \mathrm{eV}$, EI), $m / z$ (\%): 359 (100) $\left[\mathrm{M}^{+}\right], 34$ (31), 325 (15), 313 (10), 275 (95), 229 (36), 186 (30), 158 (4), 127 (23), 103 (63), 84 (16), 75 (44). IR (KBr): $\widetilde{v}=2952$ (m), 1608 (vs), 1598 (vs), 1527 (vs), 1443 (m), 1341 (s), 1248 (m), 1021 (m), 1010 (m), 844 (m), 742 (m). HRMS for $\mathbf{C}_{\mathbf{1 2}} \mathbf{H}_{13} \mathbf{I} \mathbf{N}_{\mathbf{2}} \mathbf{O}_{\mathbf{3}}$ (359.9971): found: 359.9954 .

\section{Synthesis of ethyl 2-iodo-5-nitrobenzoate (1i)}<smiles>CCOC(=O)c1cc([N+](=O)[O-])ccc1I</smiles>

Prepared according to TP 1 from $\mathrm{HNO}_{3}(100 \%, 3.8 \mathrm{~mL}), \mathrm{H}_{2} \mathrm{SO}_{4}(100 \%, 5.2 \mathrm{~mL})$ ethyl 2iodobenzoate $(11.0 \mathrm{~g}, 40.0 \mathrm{mmol})$ at $75{ }^{\circ} \mathrm{C}$. Reaction time: $12 \mathrm{~h}$. Recrystallization from ethanol furnished product $\mathbf{1} \mathbf{i}$ as pale yellow needles $(10.8 \mathrm{~g}, 84 \%)$.

mp.: $\quad 100-101.3{ }^{\circ} \mathrm{C} .{ }^{1} \mathbf{H}-\mathrm{NMR}\left(300 \mathrm{MHz}, \mathrm{CDCl}_{3}, 25^{\circ} \mathrm{C}\right): \delta=8.52\left(\mathrm{~d},{ }^{3} \mathrm{~J}(\mathrm{H}, \mathrm{H})=8.7 \mathrm{~Hz}\right.$, $1 \mathrm{H}), 8.14\left(\mathrm{~d},{ }^{4} J(\mathrm{H}, \mathrm{H})=2.7 \mathrm{~Hz}, 1 \mathrm{H}\right), 7.90\left(\mathrm{dd},{ }^{3} J(\mathrm{H}, \mathrm{H})=8.7 \mathrm{~Hz},{ }^{4} J(\mathrm{H}, \mathrm{H})=2.7 \mathrm{~Hz}, 1 \mathrm{H}\right)$, $4.39\left(\mathrm{q},{ }^{3} J(\mathrm{H}, \mathrm{H})=7.1 \mathrm{~Hz}, 2 \mathrm{H}\right), 1.38\left(\mathrm{t},{ }^{3} \mathrm{~J}(\mathrm{H}, \mathrm{H})=7.1 \mathrm{~Hz}, 3 \mathrm{H}\right) .{ }^{13} \mathbf{C}-\mathrm{NMR}\left(75 \mathrm{MHz}, \mathrm{CDCl}_{3}\right.$, $\left.25^{\circ} \mathrm{C}\right): \delta=165.0,148.1,143.1,137.1,126.6,125.8,102.9,63.0,14.6$. MS $(70 \mathrm{eV}, \mathrm{EI}), \mathrm{m} / \mathrm{z}$

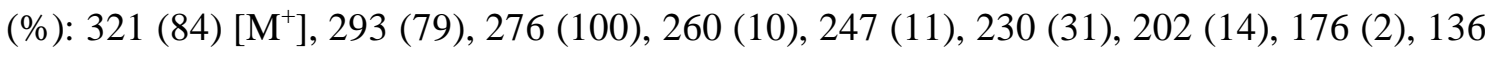
(2), 120 (5), 103 (5), 92 (9), 75 (23), 63 (3). IR (KBr): $\widetilde{v}=1731$ (vs), 1601 (s), 1565 (m), 1526 (vs), 1474 (m), 1459 (m), 1445 (m), 1384 (m), 1341 (vs), 1303 (vs), 1253 (vs), 1150 (s), 1127 (s), 1112 (m), 1091 (s), 1021 (vs), 969 (w), 919 (s), 869 (m), 837 (s), 781 (m), 734 (s). $\mathbf{C}_{9} \mathbf{H}_{8} \mathbf{I N O}_{4}$ : required: C: $33.67 ; \mathrm{H}: 2.51$; N: 4.36; I: 39.53; found: C: 33.57; H: 2.44; N: 4.29; I: 39.52 . 


\section{Synthesis of $N, N$-diethyl-2-iod-5-nitro-benzamide (1j)}

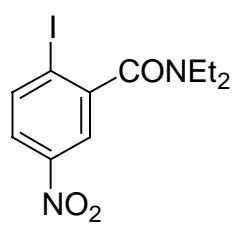

Prepared according to TP 1 from $\mathrm{HNO}_{3}(100 \%, 3.8 \mathrm{~mL}), \mathrm{H}_{2} \mathrm{SO}_{4}(100 \%, 5.2 \mathrm{~mL}) \mathrm{N}, \mathrm{N}$ diethyl-2-iodobenzamide $(11.2 \mathrm{~g}, 37.0 \mathrm{mmol})$ at $75^{\circ} \mathrm{C}$. Reaction time: $12 \mathrm{~h}$. Purification by flash-chromatography (pentane/diethyl ether $=1: 1$ ) yielded product $\mathbf{1} \mathbf{j}$ as pale yellow solid $(6.30 \mathrm{~g}, 49 \%)$.

mp.: $87-88^{\circ} \mathrm{C} .{ }^{1} \mathbf{H}-\mathbf{N M R}\left(300 \mathrm{MHz}, \mathrm{CDCl}_{3}, 25^{\circ} \mathrm{C}\right): \delta=8.04-8.00(\mathrm{~m}, 2 \mathrm{H}), 7.87$ (dd, $\left.{ }^{3} J(\mathrm{H}, \mathrm{H})=8.4 \mathrm{~Hz},{ }^{4} J(\mathrm{H}, \mathrm{H})=2.6 \mathrm{~Hz}, 1 \mathrm{H}\right), 3.92-3.70(\mathrm{~m}, 1 \mathrm{H}), 4.42-3.98(\mathrm{~m}, 3 \mathrm{H}), 1.30(\mathrm{t}$, $\left.{ }^{3} J(\mathrm{H}, \mathrm{H})=7.1 \mathrm{~Hz}, 3 \mathrm{H}\right), 1.10\left(\mathrm{t},{ }^{3} J(\mathrm{H}, \mathrm{H})=7.1 \mathrm{~Hz}, 3 \mathrm{H}\right) .{ }^{13} \mathbf{C}-\mathbf{N M R}\left(75 \mathrm{MHz}, \mathrm{CDCl}_{3}, 25{ }^{\circ} \mathrm{C}\right)$ : $\delta=169.9,148.0,144.4,140.6,124.1,121.5,101.3,43.0,39.3,14.0,12.4 . \mathrm{MS}(70 \mathrm{eV}, \mathrm{EI})$, $m / z(\%): 347$ (36) [M+], 331 (21), 319 (2), 305 (6), 276 (100), 260 (5), 248 (2), 230 (34), 218 (4), 202 (15), 175 (15), 150 (6), 131 (1), 118 (1), 104 (5), 90 (1), 75 (15), 56 (3). IR (KBr): $\tilde{v}=3280(\mathrm{~s}), 1642(\mathrm{vs}), 1602$ (m), 1550 (s), 1522 (vs), 1450 (m), 1341 (vs), 1309 (s), 1253 (w), 1160 (w), 1150 (w), 1125 (w), 1099 (w), 1047 (w), 1019 (m), 843 (m), 827 (w), 778 (w), 735 (m). HRMS for $\mathbf{C}_{\mathbf{1 1}} \mathbf{H}_{\mathbf{1 3}} \mathbf{I N}_{\mathbf{2}} \mathbf{O}_{\mathbf{3}}$ (347.9971): found: 347.9985 .

\section{Synthesis of 2-iodo-4,6-dimethyl-nitrobenzene (1k) and 5-iodo-1,3-dimethyl-2- nitrobenzene (11)}

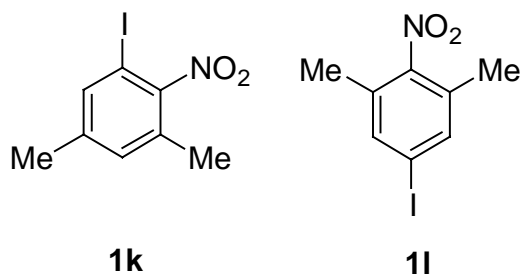

A $25 \mathrm{~mL}$ round-bottom flask, equipped with a magnetic stirring bar, was charged with $\mathrm{HNO}_{3}$ $(65 \%, 15 \mathrm{~mL})$, cooled to $0{ }^{\circ} \mathrm{C}$ and 3,5-dimethyl-iodobenzene $(4.64 \mathrm{~g}, 20.0 \mathrm{mmol})$ was added dropwise. On completion of the addition, the cooling bath was removed and the mixture was heated to $50{ }^{\circ} \mathrm{C}$ for $4 \mathrm{~h}$. The reaction mixture was poured onto ice $(20 \mathrm{~g})$, neutralized with saturated $\mathrm{NaHCO}_{3(\mathrm{aq})}$-solution and extracted with diethyl ether $(3 \times 50 \mathrm{~mL})$. The combined organic phases were washed with brine, dried over $\mathrm{Na}_{2} \mathrm{SO}_{4}$, filtered and concentrated in vacuo. Purification by flash chromatography (pentane/diethyl ether $=99: 1$ ) yielded $\mathbf{1 k}$ and $\mathbf{1 l}$ as a pale yellow solids (1k, $2.4 \mathrm{~g}, 44 \%)$ and (11, $2.3 \mathrm{~g}, 41 \%)$.

\section{Compound 1k}

mp.: $\quad 46.2-47.2{ }^{\circ} \mathrm{C} .{ }^{1} \mathbf{H}-\mathbf{N M R}\left(300 \mathrm{MHz}, \mathrm{CDCl}_{3}, 25^{\circ} \mathrm{C}\right): \delta=7.46(\mathrm{~s}, 1 \mathrm{H}), 6.97(\mathrm{~s}, 1 \mathrm{H})$, $2.23(\mathrm{~s}, 3 \mathrm{H}), 2.21(\mathrm{~s}, 3 \mathrm{H}) \cdot{ }^{13} \mathbf{C}-\mathbf{N M R}\left(75 \mathrm{MHz}, \mathrm{CDCl}_{3}, 25^{\circ} \mathrm{C}\right): \delta=153.9,142.2,138.4$, 
132.2, 131.2, 85.2, 21.1, 18.3. MS (70 eV, EI), m/z (\%): 277 (93) $\left[\mathrm{M}^{+}\right], 260$ (100), 184 (7), 133 (63), 104 (55), 91 (17), 78 (25), 63 (10), 51 (8), 43 (10). IR (KBr): $\widetilde{v}=2924$ (m), 1599 (s), 1580 (m), 1565 (s), 1529 (vs), 1448 (s), 1368 (vs), 1282 (m), 1225 (m), 1084 (m), 1039 m), 844 (vs), 792 (s), 735 (m), 728 (m), 604 (m), 588 (m). HRMS for $\mathbf{C}_{\mathbf{8}} \mathbf{H}_{\mathbf{8}} \mathbf{I N O} \mathbf{O}_{\mathbf{2}}$ (276.9600): found: 276.9588. $\mathbf{C}_{8} \mathbf{H}_{8} \mathrm{INO}_{2}$ : required: C: $34.68 ; \mathrm{H}: 2.91 ; \mathrm{N}: 5.06$; found: C: 34.74; H: 3.04; $\mathrm{N}: 5.05$.

\section{Compound 11}

mp.: $\quad 73-73.5{ }^{\circ} \mathrm{C} .{ }^{1} \mathbf{H}-\mathbf{N M R}\left(300 \mathrm{MHz}, \mathrm{CDCl}_{3}, 25^{\circ} \mathrm{C}\right): \delta=7.43(\mathrm{~s}, 2 \mathrm{H}), 2.19(\mathrm{~s}, 6 \mathrm{H}) .{ }^{\mathbf{1 3}} \mathbf{C}$ NMR $\left(75 \mathrm{MHz}, \mathrm{CDCl}_{3}, 25^{\circ} \mathrm{C}\right): \delta=151.9,138.0,131.9,96.4,17.5 . \mathrm{MS}(70 \mathrm{eV}, \mathrm{EI}), \mathrm{m} / z(\%)$ : 277 (82) [M+ $], 260$ (100), 197 (13), 184 (16), 133 (65), 104 (64), 91 (20), 78 (34), 63 (15), 50 (16), 43 (17). IR (KBr): $\tilde{v}=1594$ (s), 1563 (s), 1520 (vs), 1470 (s), 1459 (s), 1438 (s), 1409 (m), 1399 (m), 1382 (s), 1365 (vs), 1280 (s), 1260 (s), 1245 (m), 1133 (s), 1084 (m), 1035 (m), 855 (vs), 712 (m), 589 (m), 582 (m). HRMS for $\mathbf{C}_{\mathbf{8}} \mathbf{H}_{\mathbf{8}} \mathbf{I N O} \mathbf{O}_{\mathbf{2}}$ (276.9600): found: 276.9622. $\mathbf{C}_{8} \mathbf{H}_{8} \mathbf{I N O}_{2}$ : required: C: $34.68 ; \mathrm{H}: 2.91 ; \mathrm{N}: 5.06$; found: C: $34.44 ; \mathrm{H}: 2.56 ; \mathrm{N}: 4.91$.

\section{Synthesis of 1,4-diiodo-2 nitrobenzene (1m)}

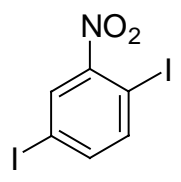

A $50 \mathrm{~mL}$ round-bottom flask, equipped with a magnetic stirring bar and a dropping funnel was charged with water $(3.8 \mathrm{~mL})$ and $\mathrm{H}_{2} \mathrm{SO}_{4}(100 \%, 3.5 \mathrm{~mL})$, glacial acetic acid $(3.5 \mathrm{~mL})$ and 4-iodo-2 nitroaniline ${ }^{1}(1.32 \mathrm{~g}, 5.00 \mathrm{mmol})$ were added at $0{ }^{\circ} \mathrm{C}$. Afterwards, a solution of $\mathrm{NaNO}_{2}(380 \mathrm{mg}, 5.5 \mathrm{mmol}$, in $1.5 \mathrm{~mL} \mathrm{H}$ O $)$ was added dropwies over a period of $1 \mathrm{~h}$. On completion of the addition the mixture was stirred for $30 \mathrm{~min}$ and a solution of KI $(1.0 \mathrm{~g}$, $6 \mathrm{mmol}$, in $1.5 \mathrm{~mL} \mathrm{H}_{2} \mathrm{O}$ ) was added dropwise with a strong evolution of gas $\left(\mathrm{N}_{2}\right)$. On completion of addition the sticky mixture was heated to $60{ }^{\circ} \mathrm{C}$ for $1 \mathrm{~h}$, cooled again to $0{ }^{\circ} \mathrm{C}$ and diethyl ether was added until all precipitate was dissolved and the reaction mixture was poured into saturated $\mathrm{NaHCO}_{3(\mathrm{aq})}$ solution $(30 \mathrm{~mL})$ in a separatory funnel. After extraction with diethyl ether $(2 \times 30 \mathrm{ml})$ the organic phases were washed with brine, dried over $\mathrm{Na}_{2} \mathrm{SO}_{4}$, filtered and concentrated in vacuo. The residue was recrystallized from ethanol to give compound $1 \mathrm{~m}$ as a yellow-orange powder $(1.27 \mathrm{~g}, 95 \%)$.

mp.: $\quad 106.5-107.2{ }^{\circ} \mathrm{C} .{ }^{1} \mathbf{H}-\mathbf{N M R}\left(300 \mathrm{MHz}, \mathrm{CDCl}_{3}, 25^{\circ} \mathrm{C}\right): \delta=8.07\left(\mathrm{~d},{ }^{4} J(\mathrm{H}, \mathrm{H})=1.8 \mathrm{~Hz}\right.$, $1 \mathrm{H}), 7.67\left(\mathrm{~d},{ }^{3} J(\mathrm{H}, \mathrm{H})=8.4 \mathrm{~Hz}, 1 \mathrm{H}\right), 7.47\left(\mathrm{dd},{ }^{3} J(\mathrm{H}, \mathrm{H})=8.4 \mathrm{~Hz},{ }^{4} J(\mathrm{H}, \mathrm{H})=1.8 \mathrm{~Hz}, 1 \mathrm{H}\right)$. ${ }^{13} \mathrm{C}-\mathrm{NMR}\left(750 \mathrm{MHz}, \mathrm{CDCl}_{3}, 25{ }^{\circ} \mathrm{C}\right): \delta=143.4,142.7,134.4,100.0,93.2,85.9 . \mathrm{MS}(70 \mathrm{eV}$, $\mathrm{EI}), m / z(\%): 375$ (100) [M+], 329 (31), 317 (4), 218 (7), 202 (21), 127 (3), 75 (39). IR (KBr): $\tilde{v}=1567(\mathrm{w}), 1544(\mathrm{w}), 1525(\mathrm{vs}), 1451(\mathrm{w}), 1350(\mathrm{~s}), 1252(\mathrm{w}), 1081(\mathrm{w}), 1016(\mathrm{~s}), 888$ (w), $865(w), 817(\mathrm{~m}), 750(\mathrm{w}), 724(\mathrm{w}) . \mathbf{C}_{\mathbf{6}} \mathbf{H}_{3} \mathbf{I}_{2} \mathbf{N O}_{2}$ : required: C: 19.22; H: 0.81; I: 67.70; N: 3.74; found: C: 19.27; H: 0.79; I: 67.66; N: 3.72. 


\section{Synthesis of 2-iodo-5-nitrobenzophenone (1n)}

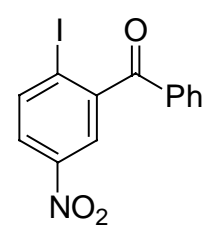

Prepared according to the procedure of Baik and Wong ${ }^{3}$ from 2-amino-5-nitrobenzophenone (726 mg, $3.00 \mathrm{mmol}), \mathrm{NaNO}_{2}(417 \mathrm{mg}, 6.00 \mathrm{mmol}), \mathrm{HI}(2.8 \mathrm{~mL}, 15 \mathrm{mmol}, 50 \%(\mathrm{aq})$ ), CuI $(570 \mathrm{mg}, 3.00 \mathrm{mmol})$ and DMSO $(20 \mathrm{~mL})$ at $60{ }^{\circ} \mathrm{C}$. Recrystallization from ethanol yielded compound $1 \mathrm{n}$ as yellow solid $(650 \mathrm{mg}, 62 \%)$.

mp.: $141.5-143{ }^{\circ} \mathrm{C} .{ }^{1} \mathbf{H}-\mathbf{N M R}\left(300 \mathrm{MHz}, \mathrm{CDCl}_{3}, 25^{\circ} \mathrm{C}\right): \delta=8.14\left(\mathrm{~d},{ }^{3} J(\mathrm{H}, \mathrm{H})=8.8 \mathrm{~Hz}\right.$, $1 \mathrm{H}), 8.10\left(\mathrm{~d},{ }^{4} J(\mathrm{H}, \mathrm{H})=2.7 \mathrm{~Hz}, 1 \mathrm{H}\right), 8.00\left(\mathrm{~d},{ }^{3} J(\mathrm{H}, \mathrm{H})=8.8 \mathrm{~Hz},{ }^{4} J(\mathrm{H}, \mathrm{H})=2.7 \mathrm{~Hz}, 1 \mathrm{H}\right), 7.81-$ $7.76(\mathrm{~m}, 2 \mathrm{H}), 7.69-7.63(\mathrm{~m}, 1 \mathrm{H}), 7.53-7.46(\mathrm{~m}, 2 \mathrm{H}) .{ }^{13} \mathbf{C}-\mathbf{N M R}\left(75 \mathrm{MHz}, \mathrm{CDCl}_{3}, 25{ }^{\circ} \mathrm{C}\right)$ : $\delta=194.9,147.7,145.9,141.1,134.5,134.5,130.4,129.0,125.1,122.8,100.6 . \mathrm{MS}(70 \mathrm{eV}$, EI), $m / z$ (\%): 353 (60) [M+ $], 275$ (9), 229 (5), 201 (3), 180 (3), 151 (6), 105 (100), 77 (43), 51 (9). IR (KBr): $\tilde{v}=1662$ (vs), 1593 (s), 1523 (vs), 1449 (m), 1350 (vs), 1315 (m), 1279 (vs), 1160 (m), 1023 (m), 967 (m), 858 (m), 850 (m), 798 (m), 761 (m), 740 (m), 728 (m), $705(\mathrm{~m})$, $688(\mathrm{~m}), 638(\mathrm{~m}) . \mathbf{H R M S}$ for $\mathbf{C}_{\mathbf{1 3}} \mathbf{H}_{\mathbf{8}} \mathbf{I N O} \mathbf{N O}_{\mathbf{3}}$ (352.9549): found: $352.9579 . \mathbf{C}_{\mathbf{1 3}} \mathbf{H}_{\mathbf{8}} \mathbf{I N O} \mathbf{O}_{\mathbf{3}}$ : required: C: 44.22; H: 2.28; N: 3.97; found: C: 44.70; H: 2.32; N: 4.03.

\section{Synthesis of 4-iodo-3-nitrobenzophenone (10)}<smiles>O=C(c1ccccc1)c1ccc(I)c([N+](=O)[O-])c1</smiles>

Prepared according to the procedure reported of Baik and Wong ${ }^{3}$ from 4-amino-3nitrobenzophenone (726 mg, $3.00 \mathrm{mmol}), \mathrm{KNO}_{2}(510 \mathrm{mg}, 6.00 \mathrm{mmol}), \mathrm{HI}$ (2.8 mL, $15 \mathrm{mmol}$, $\left.50 \%_{(\mathrm{aq})}\right), \mathrm{CuI}(570 \mathrm{mg}, 3.00 \mathrm{mmol})$ and DMSO $(20 \mathrm{~mL})$ at $60{ }^{\circ} \mathrm{C}$. Recrystallization from ethanol yielded compound 10 as yellow solid (750 mg, 73\%).

mp.: $\quad 116.7-117.3{ }^{\circ} \mathrm{C} .{ }^{1} \mathbf{H}-N M R\left(300 \mathrm{MHz}, \mathrm{CDCl}_{3}, 25^{\circ} \mathrm{C}\right): \delta=8.21-8.17(\mathrm{~m}, 2 \mathrm{H}), 7.79$ 7.75 (m, $2 \mathrm{H})$, 7.68-7.62 (m, $2 \mathrm{H}), 7.55-7.49$ (m, $2 \mathrm{H}) .{ }^{13} \mathbf{C}-\mathbf{N M R}\left(75 \mathrm{MHz}, \mathrm{CDCl}_{3}, 25{ }^{\circ} \mathrm{C}\right)$ : $\delta=193.3,153.0,142.2,138.6,135.9,133.6,133.5,129.9,128.8,126.2,91.3 . \mathrm{MS}(70 \mathrm{eV}$, $\mathrm{EI}), m / z(\%): 353$ (55) $\left[\mathrm{M}^{+}\right], 275$ (12), 229 (5), 180 (3), 152 (4), 127 (2), 105 (100), 77 (42), 51 (8). IR (KBr): $\tilde{v}=1651$ (vs), 1590 (vs), 1534 (vs), 1340 (m), 1280 (vs), 1245 (s), 1021 (m), 975 (m), 795 (w), 719 (vs), 697 (m), 672 (m). HRMS for $\mathbf{C}_{\mathbf{1 3}} \mathbf{H}_{\mathbf{8}} \mathbf{I N O}_{\mathbf{3}}$ (352.9549): found: 352.9521 . 


\section{Synthesis of 2-iodo-4-nitrophenol (1p) and 2,6-diiodo-4-nitrophenol (1e)}<smiles>O=[N+]([O-])c1ccc(O)c(I)c1</smiles><smiles>O=[N+]([O-])c1cc(I)c(O)c(I)c1</smiles>

Prepared according to TP 2 from 4-nitrophenol (1.39 g, $10.0 \mathrm{mmol})$, iodine (2.79 g, $11.0 \mathrm{mmol})$ and $\mathrm{Ag}_{2} \mathrm{SO}_{4}(3.43 \mathrm{~g}, 11.0 \mathrm{mmol})$ in ethanol $(100 \mathrm{~mL})$. Purification by flashchromatography furnished products 69 and 70 as light yellow solids; $1 \mathbf{p}(1.33 \mathrm{~g}, 50 \%)$ and $\mathbf{1 e}$ (858 mg, 22\%).

\section{Compound 1p}

mp.: $\quad 78-79{ }^{\circ} \mathrm{C} .{ }^{1} \mathbf{H}-\mathbf{N M R}\left(300 \mathrm{MHz}, \mathrm{CDCl}_{3}, 25^{\circ} \mathrm{C}\right): \delta=8.57\left(\mathrm{~d},{ }^{4} J(\mathrm{H}, \mathrm{H})=2.7 \mathrm{~Hz}, 1 \mathrm{H}\right)$, $8.15\left(\mathrm{dd},{ }^{3} J(\mathrm{H}, \mathrm{H})=8.8 \mathrm{~Hz},{ }^{4} J(\mathrm{H}, \mathrm{H})=2.7 \mathrm{~Hz}, 1 \mathrm{H}\right), 7.05\left(\mathrm{~d},{ }^{4} J(\mathrm{H}, \mathrm{H})=2.7 \mathrm{~Hz}, 1 \mathrm{H}\right), 6.00$ (s_br, $1 \mathrm{OH}) .{ }^{13} \mathrm{C}-\mathrm{NMR}\left(75 \mathrm{MHz}, \mathrm{CDCl}_{3}, 25{ }^{\circ} \mathrm{C}\right): \delta=136.4,134.4,126.1,115.7,114.6,84.4$.

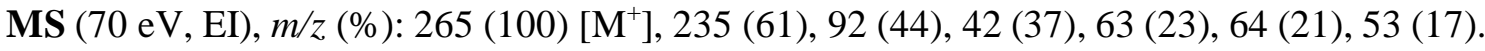
IR (KBr): $\tilde{v}=3550$ (w), 1581 (s), 1510 (vs), 1487 (s), 1414 (w), 1342 (vs), 1290 (vs), 1212 (w), 1130 (w), 1117 (s), $747(\mathrm{~s}), 683$ (m), 638 (w), 552 (w), $521(\mathrm{w}), 466(\mathrm{w}), 435(\mathrm{w})$. HRMS for $\mathbf{C}_{6} \mathbf{H}_{4} \mathbf{I N O}_{3}$ (264.9236): found: 264.9243. $\mathbf{C}_{6} \mathbf{H}_{4} \mathbf{I N O}_{3}$ : required: C: 27.19; H: 1.52; $\mathrm{N}: 5.29$; found: C: $27.53 ; \mathrm{H}: 1.82 ; \mathrm{N}: 5.22$.

\section{Compound 1e}

mp.: $\quad 155-156{ }^{\circ} \mathrm{C} .{ }^{1} \mathbf{H}-\mathbf{N M R}\left(300 \mathrm{MHz}, \mathrm{CDCl}_{3}, 25{ }^{\circ} \mathrm{C}\right): \delta=8.42 \quad(\mathrm{~s}, 2 \mathrm{H}),{ }^{\mathbf{1 3}} \mathbf{C}-\mathbf{N M R}$ $\left(75 \mathrm{MHz}, \mathrm{CDCl}_{3}, 25^{\circ} \mathrm{C}\right): \delta=160.9,141.2,134.5,82.2$. MS (70 eV, EI), $\mathrm{m} / z(\%): 391(100)$, 361 (22), 345 (3), 218 (24), 127 (11), 91 (14).

Spectral data matches that of the commercially available sample.

\section{Synthesis of 2-iodo-4-nitrophenyl 4-methylbenzenesulfonate (1q)}

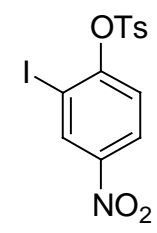

Prepared according to TP 7 from 2-iodo-4-nitrophenol (1p) $(2.85 \mathrm{~g}, 10.0 \mathrm{mmol})$ and $p$ toluenesulfonicacid chloride $(2.52 \mathrm{~g}, 12.0 \mathrm{mmol})$. Recrystallization from ethanol furnished title compound 1q as light yellow solid (3.85 g, 92\%).

mp.: $101-101.5{ }^{\circ} \mathrm{C} .{ }^{1} \mathbf{H}-\mathbf{N M R}\left(300 \mathrm{MHz}, \mathrm{CDCl}_{3}, 25^{\circ} \mathrm{C}\right): \delta=8.60\left(\mathrm{~d},{ }^{4} J(\mathrm{H}, \mathrm{H})=2.7 \mathrm{~Hz}\right.$, $1 \mathrm{H}), 8.21\left(\mathrm{dd},{ }^{3} J(\mathrm{H}, \mathrm{H})=9.3 \mathrm{~Hz},{ }^{4} J(\mathrm{H}, \mathrm{H})=2.7 \mathrm{~Hz}, 1 \mathrm{H}\right), 7.81\left(\mathrm{~d},{ }^{3} J(\mathrm{H}, \mathrm{H})=8.4 \mathrm{~Hz}, 1 \mathrm{H}\right), 7.51$ $\left(\mathrm{d},{ }^{3} J(\mathrm{H}, \mathrm{H})=9.3 \mathrm{~Hz}, 1 \mathrm{H}\right), 7.36\left(\mathrm{~d},{ }^{3} J(\mathrm{H}, \mathrm{H})=8.4 \mathrm{~Hz}, 1 \mathrm{H}\right), 2.46(\mathrm{~s}, 3 \mathrm{H}) .{ }^{13} \mathbf{C}-\mathbf{N M R}(100 \mathrm{MHz}$, $\left.\mathrm{CDCl}_{3}, 25^{\circ} \mathrm{C}\right): \delta=154.6,146.6,145.9,135.3,132.3,130.1,128.8,124.8,122.8,90.1,21.8$. 


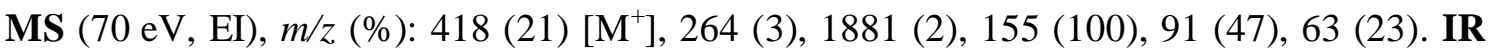
$(\mathrm{KBr}): \tilde{v}=3073(\mathrm{~m}), 1595$ (m), 1530 (vs), 1459 (s), 1381 (vs), 1348 (vs), 1291 (m), 1208 (m), 1178 (vs), 1188 (vs), 1129 (m), 1088 (m), 1035 (m), 920 (m), 877 (s), 844 (vs), 830 (m), 815 (s), 754 (vs), 733 (vs), 709 (m), 673 (vs), 650 (m), 571 (vs), 552 (m), 543 (m). HRMS for $\mathbf{C}_{13} \mathbf{H}_{10} I N O_{5} S$ (418.9324): found: 418.9285. $\mathbf{C}_{\mathbf{1 3}} \mathbf{H}_{\mathbf{1 0}} \mathbf{I N O} \mathbf{O}_{\mathbf{5}} \mathrm{S}:$ required: C: $37.25 ; \mathrm{H}: 2.40 ; \mathbf{N}$ : 3.34; S: 7.65; found: C: 37.25; H: 2.38; N: 3.37; S: 7.51 .

\section{Synthesis of 2,6-diiodo-4-nitrophenyl 4-methylbenzenesulfonate (1r)}

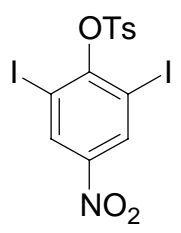

Prepared according to TP 7 from 2,6-diiodo-4-nitrophenol (1e) (3.91 g, $10.0 \mathrm{mmol})$ and $p$ toluenesulfonicacid chloride $(2.15 \mathrm{~g}, 11.0 \mathrm{mmol})$. Recrystallization from ethanol furnished title compound $\mathbf{1 r}$ as light yellow solid $(4.76 \mathrm{~g}, 90 \%)$.

mp.: $\quad 189.5-190{ }^{\circ} \mathrm{C} .{ }^{1} \mathbf{H}-\mathrm{NMR}\left(300 \mathrm{MHz}, \mathrm{CDCl}_{3}, 25^{\circ} \mathrm{C}\right): \delta=8.57$ (s, $\left.2 \mathrm{H}\right), 7.90$ (d, $\left.{ }^{3} J(\mathrm{H}, \mathrm{H})=8.4 \mathrm{~Hz}, 2 \mathrm{H}\right), 7.41\left(\mathrm{~d},{ }^{3} J(\mathrm{H}, \mathrm{H})=8.4 \mathrm{~Hz}, 2 \mathrm{H}\right), 2.42(\mathrm{~s}, 3 \mathrm{H}) .{ }^{13} \mathbf{C}-\mathbf{N M R}(75 \mathrm{MHz}$, $\left.\mathrm{CDCl}_{3}, 25^{\circ} \mathrm{C}\right): \delta=156.9,147.0,146.1,136.0,135.1,130.6,129.5,129.2,90.7,22.3 . \mathrm{MS}$

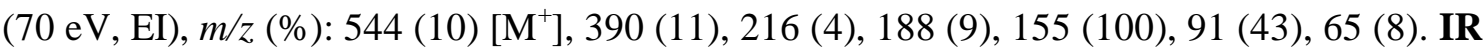
(KBr): $\tilde{v}=3088(\mathrm{w}), 1595$ (m), 1522 (vs), 1450 (w),1405 (m), 1381 (vs), 1339 (vs), 1306 (m), 1271 (w), 1216 (s), 1189 (s), 1175 (vs), 1090 (s), 1047 (m), 892 (vs), 839 (vs), 816 (s), 751 (vs), 736 (m), 723 (vs), 707 (m), 668 (vs), 631 (s), 567 (s), 544 (vs). HRMS for $\mathbf{C}_{13} \mathbf{H}_{9} \mathbf{I}_{2} \mathbf{N O}_{5} \mathbf{S}$ (544.8291): found: 544.8316. $\mathbf{C}_{13} \mathbf{H}_{9} \mathbf{I}_{2} \mathbf{N O}_{5} \mathrm{~S}$ : required: C: $28.64 ; \mathrm{H}: 1.66 ; \mathrm{N}$ : 2.57; found: C: $28.55 ; \mathrm{H}: 1.65 ; \mathrm{N}: 2.61$.

\section{Synthesis of 3,5-diiodo-2,6-dimethyl-nitrobenzene (1s)}

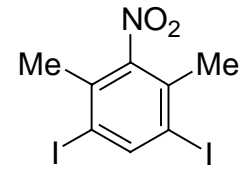

A suspension of $\mathrm{Ag}_{2} \mathrm{SO}_{4}(3.12 \mathrm{~g}, 10.0 \mathrm{mmol})$ in $\mathrm{H}_{2} \mathrm{SO}_{4}(50 \mathrm{~mL}, 90 \%(\mathrm{aq})$ was stirred at $\mathrm{rt}$ for $15 \mathrm{~min}, \mathrm{ICl}$ (3.25 g, $20.0 \mathrm{mmol}$ ) was added and stirring was continued at $\mathrm{rt}$ for $1 \mathrm{~h}$. The precipitated $\mathrm{AgCl}$ was removed by filtration and the resulting dark brown solution was added over a period of $1 \mathrm{~h}$ to a solution of 1,3-dimethyl-2-nitrobenzene $(1.41 \mathrm{~g}, 9.00 \mathrm{mmol})$, dissolved in $\mathrm{H}_{2} \mathrm{SO}_{4}\left(10 \mathrm{~mL}, 90 \%(\mathrm{aq})\right.$ at $0{ }^{\circ} \mathrm{C}$. On completion of the addition, the reaction mixture was stirred for $1 \mathrm{~h}$ at $\mathrm{rt}$ and the mixture was poured into $\mathrm{H}_{2} \mathrm{O}(100 \mathrm{~mL})$. Filtration and subsequent recrystallization from ethanol furnished compound $1 \mathrm{~s}$ as white solid $(2.73 \mathrm{~g}$, $68 \%)$. 
mp.: $\quad 137.5-139{ }^{\circ} \mathrm{C} .{ }^{1} \mathbf{H}-\mathrm{NMR}\left(300 \mathrm{MHz}, \mathrm{CDCl}_{3}, 25^{\circ} \mathrm{C}\right): \delta=8.31$ (s, $\left.2 \mathrm{H}\right), 2.24(\mathrm{~s}, 6 \mathrm{H})$. ${ }^{13}$ C-NMR $\left(75 \mathrm{MHz}, \mathrm{CDCl}_{3}, 25^{\circ} \mathrm{C}\right): \delta=148.5,138.1,130.4,97.1,21.3$. MS (70 eV, EI), $\mathrm{m} / \mathrm{z}$ (\%): 402 (74) [ $\left.\mathrm{M}^{+}\right], 385$ (95), 357 (21), 293 (9), 258 (28), 230 (36), 132 (19), 103 (100). IR $(\mathrm{KBr}): \tilde{v}=1528$ (vs), $1422(\mathrm{~m}), 1381$ (s), 1346 (m), $942(\mathrm{w}), 879$ (m), 739 (s). HRMS for $\mathbf{C}_{8} \mathbf{H}_{7} \mathbf{I}_{2} \mathbf{N O}_{2}$ (402.8566): found: 402.8575 .

\section{Synthesis of di(tert-butyl) 2-iodo-4-nitrophenylimidodicarbonate (1t) ${ }^{4}$}

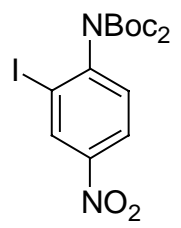

A dry and nitrogen flushed $50 \mathrm{~mL}$ Schlenk flask, equipped with a magnetic stirring bar, was charged with 2-iodo-4-nitroaniline (1d) $(2.64 \mathrm{~g}, 10.0 \mathrm{mmol})$ and acetonitrile $(25 \mathrm{~mL})$, cooled to $0{ }^{\circ} \mathrm{C}$ and DMAP $(125 \mathrm{mg}, 1.00 \mathrm{mmol})$ was added. Subsequently, a solution of $\mathrm{Boc}_{2} \mathrm{O}$ $(5.23 \mathrm{~g}, 24.0 \mathrm{mmol})$ dissolved in acetonitrile $(15 \mathrm{~mL})$ was added dropwise. On completion of addition, the cooling bath was removed, the reaction mixture stirred for $24 \mathrm{~h}$ at $60{ }^{\circ} \mathrm{C}$ and poured into water. After extraction with ethyl acetate $(3 \times 30 \mathrm{~mL})$, the combined organic fractions were washed with $\mathrm{KHSO}_{4(\mathrm{aq})}(30 \mathrm{~mL}, 1 \mathrm{M})$, brine $(30 \mathrm{~mL})$, dried over $\mathrm{MgSO}_{4}$, filtered and concentrated in vacuo. Purification by flash chromatography (pentane/diethyl ether $=9: 1)$ furnished title compound $\mathbf{1 t}$ as colourless oil $(3.41 \mathrm{~g}, 70 \%)$.

${ }^{1} \mathbf{H}-\mathbf{N M R}\left(300 \mathrm{MHz}, \mathrm{CDCl}_{3}, 25{ }^{\circ} \mathrm{C}\right): \delta=8.68\left(\mathrm{~d},{ }^{4} J(\mathrm{H}, \mathrm{H})=2.7 \mathrm{~Hz}, 1 \mathrm{H}\right), 8.22(\mathrm{dd}$, $\left.{ }^{3} J(\mathrm{H}, \mathrm{H})=8.4 \mathrm{~Hz},{ }^{4} J(\mathrm{H}, \mathrm{H})=2.7 \mathrm{~Hz}, 1 \mathrm{H}\right), 7.36\left(\mathrm{~d},{ }^{3} J(\mathrm{H}, \mathrm{H})=8.4 \mathrm{~Hz}, 1 \mathrm{H}\right), 1.40(\mathrm{~s}, 18 \mathrm{H}) .{ }^{13} \mathrm{C}-$ NMR $\left(75 \mathrm{MHz}, \mathrm{CDCl}_{3}, 25^{\circ} \mathrm{C}\right): \delta=149.2,148.0,146.7,134.1,129.4,123.9,99.7,84.0,41.8$, 27.9. MS (FAB, NBA): m/z (\%): $487[\mathrm{M}+\mathrm{Na}]^{+}$. MS (70 eV, EI), $m / z(\%): 363$ (11) [M-Boc] $]^{+}$, 307 (17), 263 (6), 233 (4), 181 (4), 91 (3.6, 57 (100). IR (KBr): $\widetilde{v}=3435$ (m), 1797 (m), 1756 (vs), 1725 (s), 1524 (vs), 1372 (m), 1346 (vs), 1315 (m), 1280 (vs), 1252 (s), 1153 (s), 1115 (vs), 860 (w), 772 (w). $\mathbf{H R M S}$ for $\mathbf{C}_{\mathbf{1 6}} \mathbf{H}_{\mathbf{2 1}} \mathbf{I} \mathbf{N}_{\mathbf{2}} \mathbf{O}_{\mathbf{6}}\left(487.0342[\mathrm{M}+\mathrm{Na}]^{+}\right)$: found: 487.0351 $[\mathrm{M}+\mathrm{Na}]^{+} . \mathbf{C}_{\mathbf{1 6}} \mathbf{H}_{21} \mathbf{I N}_{\mathbf{2}} \mathbf{O}_{6}$ : required: C: 41.39; H: 4.56; N: 6.03; found: C: 41.17; H: 4.58; N: 6.01 .

\section{Synthesis of 1-[(E)-(2-iodo-4-nitrophenyl)diazenyl]pyrrolidine (1u)}

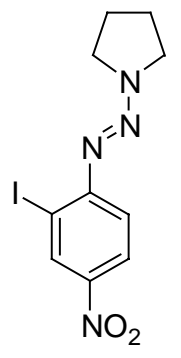


A $50 \mathrm{~mL}$ round-bottom flask, equipped with a magnetic stirring bar and a dropping funnel was charged with water $(5 \mathrm{~mL})$, conc. $\mathrm{HCl}(0.6 \mathrm{~mL})$ and 2-iodo-4-nitroaniline (1d) $(2.64 \mathrm{~g}$, $10.0 \mathrm{mmol})$ were added at $0{ }^{\circ} \mathrm{C}$. Afterwards, a solution of $\mathrm{NaNO}_{2}(825 \mathrm{mg}, 12.0 \mathrm{mmol}$, in $10 \mathrm{~mL} \mathrm{H}_{2} \mathrm{O}$ ) was added dropwise over a period of $30 \mathrm{~min}$. On completion of the addition the mixture was stirred for $30 \mathrm{~min}$ and was poured into a solution of pyrrolidine $(1.06 \mathrm{~g}$, $15.0 \mathrm{mmol}$, in $\left.9 \mathrm{~mL} \mathrm{H} \mathrm{H}_{2} \mathrm{O}\right)$ and $\mathrm{K}_{2} \mathrm{CO}_{3}(2.49 \mathrm{~g}, 18.0 \mathrm{mmol})$ with a strong evolution of gas. On completion of addition the sticky mixture was stirred for an additional $1 \mathrm{~h}$ at $\mathrm{rt}$ and the reaction mixture was poured into saturated $\mathrm{NaHCO}_{3(\mathrm{aq})}$ solution $(30 \mathrm{~mL})$ in a separatory funnel. After extraction with ethyl acetate $(2 \times 30 \mathrm{ml})$ the organic phases were washed with brine, dried over $\mathrm{Na}_{2} \mathrm{SO}_{4}$, filtered and concentrated in vacuo. The residue was recrystallized from ethanol furnishing compound $1 \mathbf{u}$ as an orange powder $(2.69 \mathrm{~g}, 78 \%)$.

mp.: $\quad 156-157{ }^{\circ} \mathrm{C} .{ }^{1} \mathbf{H}-\mathbf{N M R}\left(300 \mathrm{MHz}, \mathrm{CDCl}_{3}, 25^{\circ} \mathrm{C}\right): \delta=8.69\left(\mathrm{~d},{ }^{4} \mathrm{~J}(\mathrm{H}, \mathrm{H})=2.7 \mathrm{~Hz}, 1 \mathrm{H}\right)$, $8.12\left(\mathrm{dd},{ }^{3} J(\mathrm{H}, \mathrm{H})=8.9 \mathrm{~Hz},{ }^{4} J(\mathrm{H}, \mathrm{H})=2.7 \mathrm{~Hz}, 1 \mathrm{H}\right), 7.38\left(\mathrm{~d},{ }^{4} J(\mathrm{H}, \mathrm{H})=8.9 \mathrm{~Hz}, 1 \mathrm{H}\right), 4.00\left(\mathrm{br} \_\mathrm{t}\right.$, $\left.{ }^{3} J(\mathrm{H}, \mathrm{H})=6.6 \mathrm{~Hz}, 2 \mathrm{H}\right), 3.79$ (br_t, $\left.{ }^{3} J(\mathrm{H}, \mathrm{H})=6.6 \mathrm{~Hz}, 2 \mathrm{H}\right), 2.14-2.05(\mathrm{~m}, 4 \mathrm{H}) .{ }^{13} \mathbf{C}-\mathbf{N M R}$ $\left(75 \mathrm{MHz}, \mathrm{CDCl}_{3}, 25^{\circ} \mathrm{C}\right): \delta=155.4,144.5,134.9,124.2,116.2,94.8,51.7,48.0,24.0,23.4$. MS (70 eV, EI), m/z (\%): 346 (24) [M+ ], 275 (64), 247 (100), 217 (11), 201 (15), 75 (37), 70 (15). IR (KBr): $\tilde{v}=1505$ (s), 1406 (m), 1375 (m), 1331 (vs), 1299 (vs), 1270 (vs), 1245 (m), 1112 (m), 866 (w), 854 (w), 826 (w), 748 (w). HRMS for $\mathbf{C}_{\mathbf{1 0}} \mathbf{H}_{\mathbf{1 1}} \mathbf{I} \mathbf{N}_{\mathbf{4}} \mathbf{O}_{\mathbf{2}}$ (345.9927): found: 345.9892. $\mathbf{C}_{10} \mathbf{H}_{11} \mathbf{I N}_{\mathbf{4}} \mathbf{O}_{2}$ : required: C: $34.70 ; \mathrm{H}: 3.20 ; \mathrm{N}: 16.19$; found: C: $34.59 ; \mathrm{H}: 3.22 ; \mathrm{N}$ : 16.09.

\section{Synthesis of heterocyclic iodines 5}

\section{Synthesis of 2-iodo-3-nitropyridine (5a)}

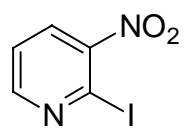

Prepared according to the procedure reported of Baik and Wong ${ }^{3}$ from 2-amino-3nitropyridine $(696 \mathrm{mg}, 5.00 \mathrm{mmol}), \mathrm{KNO}_{2}(850 \mathrm{mg}, 10.0 \mathrm{mmol}), \mathrm{HI}(3.8 \mathrm{~mL}, 25 \mathrm{mmol}$, $\left.50 \%_{(\mathrm{aq})}\right)$, CuI $(475 \mathrm{mg}, 2.50 \mathrm{mmol})$ and DMSO $(45 \mathrm{~mL})$ at $60{ }^{\circ} \mathrm{C}$. Purification by flash chromatography (pentane/diethyl ether $=2: 1$ ) yielded compound $\mathbf{5 a}$ as yellow solid $(784 \mathrm{mg}$, $63 \%)$.

mp.: $\quad 130.2-131.8{ }^{\circ} \mathrm{C} .{ }^{1} \mathbf{H}-\mathbf{N M R}\left(300 \mathrm{MHz}, \mathrm{CDCl}_{3}, 25^{\circ} \mathrm{C}\right): \delta=8.55\left(\mathrm{dd},{ }^{3} J(\mathrm{H}, \mathrm{H})=4.9 \mathrm{~Hz}\right.$, $\left.{ }^{4} J(\mathrm{H}, \mathrm{H})=1.8 \mathrm{~Hz}, 1 \mathrm{H}\right), 8.05\left(\mathrm{dd},{ }^{3} J(\mathrm{H}, \mathrm{H})=8.0 \mathrm{~Hz},{ }^{4} J(\mathrm{H}, \mathrm{H})=1.8 \mathrm{~Hz}, 1 \mathrm{H}\right), 7.50 \quad(\mathrm{dd}$, $\left.{ }^{3} J(\mathrm{H}, \mathrm{H})=8.0 \mathrm{~Hz},{ }^{3} J(\mathrm{H}, \mathrm{H})=4.9 \mathrm{~Hz}, 1 \mathrm{H}\right) .{ }^{13} \mathbf{C}-\mathbf{N M R}\left(75 \mathrm{MHz}, \mathrm{CDCl}_{3}, 25{ }^{\circ} \mathrm{C}\right): \delta=153.4$, 151.0, 132.8, 123.4, 110.4. MS (70 eV, EI), m/z (\%): 249 (100) $\left[\mathrm{M}^{+}\right], 203$ (34), 126 (8), 77 (22), 53 (22). IR (KBr): $\widetilde{v}=3037$ (m), 1574 (vs), 1555 (vs), 1526 (vs), 1447 (w), 1401 (vs), 1347 (vs), 1245 (m), 1221 (m), 1053 (m), 1039 (s), 854 (s), 827 (s), 740 (s), 720 (s), 633 (m), 565 (w). HRMS for $\mathbf{C}_{\mathbf{1 2}} \mathbf{H}_{\mathbf{1 0}} \mathbf{N}_{\mathbf{2}} \mathbf{O}_{\mathbf{2}}$ (249.9239): found: 249.9208. $\mathbf{C}_{5} \mathbf{H}_{\mathbf{3}} \mathbf{I} \mathbf{N}_{\mathbf{2}} \mathbf{O}_{2}$ : required: $\mathrm{C}$ : 24.02; H: 1.21; N: 11.21; found: C: 24.13; H: 1.08; N: 11.18 . 


\section{Synthesis of 5-iodo-6-nitroquinoline (5b)}

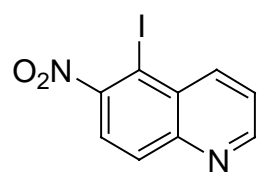

Prepared according to the procedure reported of Baik and Wong ${ }^{3}$ from 5-amino-6nitroquinoline (946 mg, $5.00 \mathrm{mmol}), \mathrm{KNO}_{2}(935 \mathrm{mg}, 11.0 \mathrm{mmol}), \mathrm{HI}$ (3.9 mL, $27 \mathrm{mmol}$, $\left.50 \%_{(\mathrm{aq})}\right) \mathrm{CuI}(950 \mathrm{mg}, 5.00 \mathrm{mmol})$ and DMSO $(45 \mathrm{~mL})$ at $60{ }^{\circ} \mathrm{C}$. Purification by flash chromatography (pentane/diethyl ether $=2: 1$ ) yielded compound $\mathbf{5 b}$ as green-yellow solid $(1.26 \mathrm{~g}, 84 \%)$.

mp.: $159.7-161.9{ }^{\circ} \mathrm{C} .{ }^{1} \mathbf{H}-\mathrm{NMR}\left(300 \mathrm{MHz}, \mathrm{CDCl}_{3}, 25^{\circ} \mathrm{C}\right): \delta=9.01$ (s_br, $\left.1 \mathrm{H}\right), 8.66$ (d, $\left.{ }^{3} J(\mathrm{H}, \mathrm{H})=8.4 \mathrm{~Hz}, 1 \mathrm{H}\right), 8.21\left(\mathrm{~d},{ }^{3} J(\mathrm{H}, \mathrm{H})=9.3 \mathrm{~Hz}, 1 \mathrm{H}\right), 8.91\left(\mathrm{~d},{ }^{3} J(\mathrm{H}, \mathrm{H})=8.9 \mathrm{~Hz}, 1 \mathrm{H}\right), 7.50$ $\left(\mathrm{dd},{ }^{3} J(\mathrm{H}, \mathrm{H})=8.9 \mathrm{~Hz},{ }^{3} J(\mathrm{H}, \mathrm{H})=4.5 \mathrm{~Hz}, 1 \mathrm{H}\right),{ }^{13} \mathrm{C}-\mathbf{N M R} \quad\left(150 \mathrm{MHz}, \mathrm{CDCl}_{3}, \quad 25^{\circ} \mathrm{C}\right)$ : $\delta=153.3,153.2,148.5,142.9,131.9,129.8,124.5,124.2,92.8 . \mathbf{M S}(70 \mathrm{eV}, \mathrm{EI}), \mathrm{m} / z(\%): 300$ (100) $[\mathrm{M}]^{+}, 254$ (31), 242 (15), 145 (18), 127 (44), 115 (14), 101 (11), 74 (7). IR (KBr): $\widetilde{V}=1597(\mathrm{w}), 1561$ (vs), 1524 (vs), 1488 (s), 1335 (s), 1325 (s), 1307 (m), 1299 (m), 963 (m), 838 (m), 806 (s), 772 (m), 731 (w). HRMS for $\mathbf{C}_{\mathbf{9}} \mathbf{H}_{5} \mathbf{I} \mathbf{I N}_{\mathbf{2}} \mathbf{O}_{\mathbf{2}}$ (299.9396): found: 299.9409.

\section{Synthesis of compounds 9}

\section{Synthesis of (2-nitrophenyl)(phenyl)methanol (9a)}<smiles>O=[N+]([O-])c1ccccc1C(O)c1ccccc1</smiles>

Prepared according to TP 3 from 2-iodonitrobenzene (1a) (498 mg, $2.00 \mathrm{mmol}) \mathrm{PhMgCl}$ $(1.1 \mathrm{~mL}, 2.2 \mathrm{mmol}, 2.0 \mathrm{M}$ in THF) and benzaldehyde $(255 \mathrm{mg}, 2.40 \mathrm{mmol})$. Reaction time: $0.5 \mathrm{~h}$. Purification by flash chromatography (pentane/diethyl ether $=4: 1$ ) yielded the benzylic alcohol 9a as a red oil $(400 \mathrm{mg}, 87 \%)$.

${ }^{1} \mathbf{H}$-NMR $\left(300 \mathrm{MHz}, \mathrm{CDCl}_{3}, 25^{\circ} \mathrm{C}\right): \delta=7.84\left(\mathrm{dd},{ }^{3} J(\mathrm{H}, \mathrm{H})=8.4 \mathrm{~Hz},{ }^{4} J(\mathrm{H}, \mathrm{H})=1.4 \mathrm{~Hz}, 1 \mathrm{H}\right)$, $7.65\left(\mathrm{dd}, \quad{ }^{3} J(\mathrm{H}, \mathrm{H})=8.4 \mathrm{~Hz}, \quad{ }^{4} J(\mathrm{H}, \mathrm{H})=1.4 \mathrm{~Hz}, \quad 1 \mathrm{H}\right), \quad 7.54 \quad\left(\mathrm{dt}, \quad{ }^{3} J(\mathrm{H}, \mathrm{H})=8.4 \mathrm{~Hz}\right.$, $\left.{ }^{4} J(\mathrm{H}, \mathrm{H})=1.3 \mathrm{~Hz}, 1 \mathrm{H}\right), 7.36\left(\mathrm{dt},{ }^{3} J(\mathrm{H}, \mathrm{H})=8.6 \mathrm{~Hz},{ }^{4} J(\mathrm{H}, \mathrm{H})=1.3 \mathrm{~Hz}, 1 \mathrm{H}\right), 7.25-7.17(\mathrm{~m}$, $5 \mathrm{H}), 6.34$ (s_br, $1 \mathrm{H}), 2.83$ (s_br, $1 \mathrm{OH}) .{ }^{13} \mathrm{C}-\mathbf{N M R}\left(75 \mathrm{MHz}, \mathrm{CDCl}_{3}, 25{ }^{\circ} \mathrm{C}\right): \delta=148.8$, $141.9,138.9,133.8,129.8,129.0,128.4,127.3,126.9,125.1,71.9 . \mathbf{M S}(70 \mathrm{eV}, \mathrm{EI}), \mathrm{m} / z(\%)$ : $229(0.1)\left[\mathrm{M}^{+}\right], 211$ (5), 194 (46), 181 (21), 167 (100), 152 (57), 39 (10), 127 (5), 115 (5), 105 (17), 92 (2), 77 (46), 65 (4), 51 (12). IR (KBr): $\tilde{v}=3412$ (m, br), 3064 (m), 1955 (w), 1740 (w), 1667 (w), 1598 (m), 1578 (m), 1525 (vs), 1452 (s), 1349 (vs), 1260 (s), 1178 (s), 1022 (s), 861 (m), 787 (m), 760 (s), 736 (s), 701 (vs), 647 (w), 601 (w). HRMS for $\mathbf{C}_{\mathbf{1 3}} \mathbf{H}_{\mathbf{1 1}} \mathbf{N O}_{\mathbf{3}}$ (229.0739): found: 229.0518 . 


\section{Synthesis of (2,4-dinitrophenyl)(phenyl)methanol (9b)}

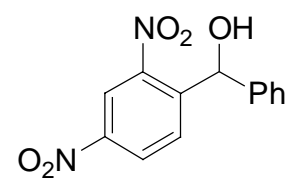

Prepared according to TP 3 from 2,4-dinitro-iodobenzene (1b) (588 mg, $2.00 \mathrm{mmol}$ ), PhMgCl $(1.1 \mathrm{~mL}, 2.1 \mathrm{mmol}, 2.0 \mathrm{M}$ in THF) and benzaldehyde (424 mg, $4.00 \mathrm{mmol})$. Reaction time: $0.5 \mathrm{~h}$. Purification by flash chromatography $\left(\mathrm{CH}_{2} \mathrm{Cl}_{2}\right)$ yielded compound $\mathbf{9 b}(442 \mathrm{mg}, 81 \%)$ as an orange oil.

${ }^{1} \mathbf{H}-\mathbf{N M R}\left(300 \mathrm{MHz}, \mathrm{CDCl}_{3}, 25^{\circ} \mathrm{C}\right): \delta=8.66\left(\mathrm{~d},{ }^{4} J(\mathrm{H}, \mathrm{H})=2.1 \mathrm{~Hz}, 1 \mathrm{H}\right), 8.39(\mathrm{dd}$, $\left.{ }^{3} J(\mathrm{H}, \mathrm{H})=8.4 \mathrm{~Hz},{ }^{4} J(\mathrm{H}, \mathrm{H})=2.1 \mathrm{~Hz}, 1 \mathrm{H}\right), 8.07\left(\mathrm{~d},{ }^{3} J(\mathrm{H}, \mathrm{H})=8.4 \mathrm{~Hz}, 1 \mathrm{H}\right), 7.30-7.18(\mathrm{~m}, 5 \mathrm{H})$, 6.47 (s, $1 \mathrm{H}), 2,46$ (s_br, $1 \mathrm{OH}) .{ }^{13} \mathbf{C}-\mathbf{N M R}\left(75 \mathrm{MHz}, \mathrm{CDCl}_{3}, 25{ }^{\circ} \mathrm{C}\right): \delta=146.9,146.1,143.9$, 139.3, 129.4, 128.0, 127.8, 126.2, 126.1, 119.2, 70.3. MS (70 eV, EI), m/z (\%): $256(11)$ $\left[\mathrm{M}-\mathrm{H}_{2} \mathrm{O}\right]^{+}, 239$ (100), 210 (17), 193 (15), 180 (25), 166 (85), 152 (68), 139 (32), 127 (11), 105 (31), 77 (76), 63 (8), 51 (14). IR (KBr): $\tilde{v}=3542$ (s), 3111 (s), 1605 (s), 1538 (vs), 1454 (s), 1348 (vs), 1176 (m), 1036 (s), 919 (m), 836 (s), 758 (s), 740 (s), 701 (s). HRMS for $\mathbf{C}_{13} \mathbf{H}_{10} \mathbf{N}_{\mathbf{2}} \mathbf{O}_{5}$ (274.0590): found: $272.0432[\mathrm{M}-2 \mathrm{H}]^{+}$. $\mathbf{C}_{\mathbf{1 3}} \mathbf{H}_{\mathbf{1 0}} \mathbf{N}_{\mathbf{2}} \mathbf{O}_{\mathbf{5}}$ : required: C: $56.94, \mathrm{H}$ : 3.68, N: 10.22; found: C: $57.13, \mathrm{H}: 3.71, \mathrm{~N}: 10.01$.

\section{Synthesis of cyclohexyl(2,4-dinitrophenyl)methanol (9c)}

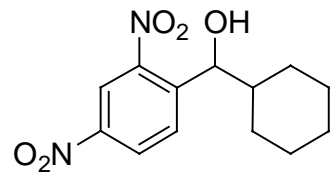

Prepared according to TP 3 from 2,4-dinitro-iodobenzene (1b) (588 mg, $2.00 \mathrm{mmol}), \mathrm{PhMgCl}$ (1.1 mL, $2.1 \mathrm{mmol}, 2 \mathrm{M}$ in THF) and cyclohexanecarbaldehyde (560 mg, $5.00 \mathrm{mmol}$ ). Reaction time: $0.5 \mathrm{~h}$. Purification by flash chromatography $\left(\mathrm{CH}_{2} \mathrm{Cl}_{2} /\right.$ pentane $\left.=4: 1\right)$ yielded product $9 \mathrm{c}(416 \mathrm{mg}, 74 \%)$ as an orange oil

${ }^{1} \mathbf{H}$-NMR $\left(300 \mathrm{MHz}, \mathrm{CDCl}_{3}, 25{ }^{\circ} \mathrm{C}\right): \delta=8.67\left(\mathrm{~d},{ }^{4} J(\mathrm{H}, \mathrm{H})=2.1 \mathrm{~Hz}, 1 \mathrm{H}\right), 8.37 \quad(\mathrm{dd}$, $\left.{ }^{3} J(\mathrm{H}, \mathrm{H})=8.4 \mathrm{~Hz}, \quad{ }^{4} J(\mathrm{H}, \mathrm{H})=2.1 \mathrm{~Hz}, \quad 1 \mathrm{H}\right), 7.61 \quad\left(\mathrm{~d},{ }^{3} J(\mathrm{H}, \mathrm{H})=8.4 \mathrm{~Hz}, 1 \mathrm{H}\right), \quad 5.19 \quad(\mathrm{~d}$, $\left.{ }^{3} J(\mathrm{H}, \mathrm{H})=5 \mathrm{~Hz}, 1 \mathrm{H}\right), 2.18\left(\mathrm{~s} \_b r, 1 \mathrm{OH}\right), 1.69-157(\mathrm{~m}, 5 \mathrm{H}), 1.44-1.41(\mathrm{~m}, 1 \mathrm{H}), 1.18-1.03$ (m, $5 \mathrm{H}) .{ }^{13} \mathrm{C}$-NMR $\left(75 \mathrm{MHz}, \mathrm{CDCl}_{3}, 2{ }^{\circ} \mathrm{C}\right): \delta=148.3,147.1,146.1,131.1,127.1,120.2,73.3$, 44.8, 30.2, 27.2, 26.5, 26.4, 26.2. MS (70 eV, EI), m/z (\%): 261 (2) $\left[\mathrm{M}-\mathrm{H}_{2} \mathrm{O}\right]^{+}, 245$ (5), 217 (4), 198 (90), 180 (100), 164 (8), 151 (14), 134 (31), 124 (5), 103 (9), 83 (50), 77 (13), 67 (7), 55 (75). IR (KBr): $\quad \tilde{v}=3562$ (br, s), 3111 (m), 2926 (vs), 1605 (m), 1538 (vs), 1450 (s), 1353 (vs), 1262 (m), 1188 (m), 1083 (s), 1024 (m), 916 (m), 836 (s), 745 (s), 720 (s). HRMS for $\mathbf{C}_{\mathbf{1 3}} \mathbf{H}_{16} \mathbf{N}_{\mathbf{2}} \mathbf{O}_{\mathbf{5}}$ (280.1059): found: 263.0999[M-OH] ${ }^{+}$. $\mathbf{C}_{\mathbf{1 3}} \mathbf{H}_{\mathbf{1 6}} \mathbf{N}_{\mathbf{2}} \mathbf{O}_{\mathbf{5}}$ : required: C: $55.71, \mathbf{H}$ : 5.75, N: 9.99; found: C: 55.60, H: 5.78, N: 9.62. 


\section{Synthesis of (4-methoxy-2-nitrophenyl)(phenyl)methanol (9d)}

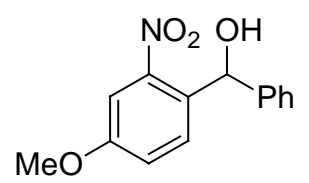

Prepared according to TP 3 from 1-iodo-4-methoxy-2-nitrobenzene (1c) (418 mg, $1.50 \mathrm{mmol}), \mathrm{PhMgCl}(1.0 \mathrm{~mL}, 1.7 \mathrm{mmol}, 1.7 \mathrm{M}$ in $\mathrm{THF})$ and benzaldehyde (190 mg, $1.80 \mathrm{mmol})$. Reaction time: $0.5 \mathrm{~h}$. Purification by flash chromatography $\left(\mathrm{CH}_{2} \mathrm{Cl}_{2}\right)$ yielded (4methoxy-2-nitrophenyl)(phenyl)methanol (9d) (279 mg, 72\%) as a yellow oil.

${ }^{1} \mathbf{H}$-NMR $\quad\left(300 \mathrm{MHz}, \quad \mathrm{CDCl}_{3}, \quad 25^{\circ} \mathrm{C}\right): \quad \delta=7.48 \quad\left(\mathrm{~d}, \quad{ }^{3} J(\mathrm{H}, \mathrm{H})=8.5 \mathrm{~Hz}, \quad 1 \mathrm{H}\right), \quad 7.35(\mathrm{~d}$, $\left.{ }^{4} J(\mathrm{H}, \mathrm{H})=1.9 \mathrm{~Hz}, 1 \mathrm{H}\right), 7.26-7.18(\mathrm{~m}, 5 \mathrm{H}), 7.05\left(\mathrm{dd},{ }^{3} J(\mathrm{H}, \mathrm{H})=8.5 \mathrm{~Hz},{ }^{4} J(\mathrm{H}, \mathrm{H})=1.9 \mathrm{~Hz}\right.$, $1 \mathrm{H}), 6.25$ (s, $1 \mathrm{H}), 3.78$ (s, $3 \mathrm{H}), 2.61$ (s_br, $1 \mathrm{OH}) .{ }^{13} \mathbf{C}-\mathbf{N M R}\left(75 \mathrm{MHz}, \mathrm{CDCl}_{3}, 25{ }^{\circ} \mathrm{C}\right)$ : $\delta=159.6,149.4,142.2,131.1,131.0,128.9,128.2,127.2,120.1,109.8,71.6,56.3$. MS $(70 \mathrm{eV}, \mathrm{EI}), m / z(\%): 241(26)\left[\mathrm{M}-\mathrm{H}_{2} \mathrm{O}\right]^{+}, 224$ (48), 211 (27), 196 (32), 182 (100), 168 (50), 154 (87), 140 (22), 135 (26), 127 (11), 115 (18), 105 (22), 92 (12), 77 (80), 63 (18), 51 (17). IR (KBr): $\tilde{v}=3400(\mathrm{~m}), 2840$ (w), 1621 (m), 1530 (vs), 1352 (s), 1244 (s), 1181 (m), 1066 (m), 1033 (s), 759 (m), 700 (s), 549 (w). $\mathbf{C}_{\mathbf{1 4}} \mathbf{H}_{\mathbf{1 3}} \mathbf{N O}_{\mathbf{4}}$ : required: C: 64.86, H: 5.40, N: 5.40; found: C: $65.22, \mathrm{H}: 5.13, \mathrm{~N}: 5.23$.

\section{Synthesis of 1-(4-methoxy-2-nitrophenyl)-1-hexanol (9e)}

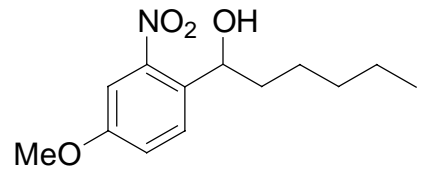

Prepared according to TP 3 from 3-iodo-4-nitroanisole (1c) (418 mg, $1.50 \mathrm{mmol}), \mathrm{PhMgCl}$ $(0.85 \mathrm{~mL}, 1.6 \mathrm{mmol}, 1.9 \mathrm{M}$ in THF) and hexanal (201 mg, $2.00 \mathrm{mmol})$. Reaction time: $2 \mathrm{~h}$. Purification by flash chromatography (pentane/diethyl ether $=7: 1$ ) yielded product 9e (270 $\mathrm{mg}, 71 \%$ ) as a colourless oil.

${ }^{1} \mathbf{H}-\mathbf{N M R} \quad\left(300 \mathrm{MHz}, \quad \mathrm{CDCl}_{3}, \quad 25{ }^{\circ} \mathrm{C}\right): \delta=7.60 \quad\left(\mathrm{~d}, \quad{ }^{3} J(\mathrm{H}, \mathrm{H})=8.4 \mathrm{~Hz}, \quad 1 \mathrm{H}\right), \quad 7.29 \quad(\mathrm{~d}$, $\left.{ }^{4} J(\mathrm{H}, \mathrm{H})=1.9 \mathrm{~Hz}, 1 \mathrm{H}\right), 7.06\left(\mathrm{dd},{ }^{3} J(\mathrm{H}, \mathrm{H})=8.4 \mathrm{~Hz},{ }^{4} J(\mathrm{H}, \mathrm{H})=1.9 \mathrm{~Hz}, 1 \mathrm{H}\right), 5.07-5.03(\mathrm{~m}$, $1 \mathrm{H}), 3.78$ (s, $3 \mathrm{H}), 2.37$ (s_br, $1 \mathrm{OH}), 1.68-1.21$ (m, $8 \mathrm{H}), 0.83-0.78$ (m, $3 \mathrm{H}) .{ }^{13} \mathbf{C}-\mathbf{N M R}$ $\left(75 \mathrm{MHz}, \mathrm{CDCl}_{3}, 25^{\circ} \mathrm{C}\right): \delta=159.2,148.9,132.6,129.5,120.4,109.1,69.4,56.2,38.4,31.9$,

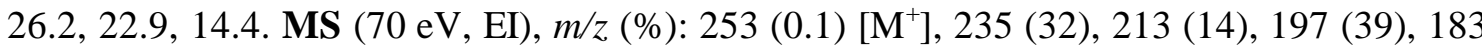
(100), 170 (28), 153 (81), 140 (14), 135 (13), 126 (22), 115 (25), 77 (5), 63 (3) (43 (26). IR $(\mathrm{KBr}): \tilde{v}=3401(\mathrm{~m}), 2956(\mathrm{~s}), 1621(\mathrm{~m}), 1530(\mathrm{vs}), 1498(\mathrm{~m}), 1463(\mathrm{~m}), 1351(\mathrm{~s}), 1297(\mathrm{~m})$, 1242 (s), 1184 (m), 1119 (w), 1066 (m), 1037 (s), 929 (w), 854 (w), 832 (w), 761 (w). HRMS for $\mathbf{C}_{13} \mathbf{H}_{19} \mathrm{NO}_{4}$ (253.1314): found: $254.1136[\mathrm{M}+\mathrm{H}]^{+}$. 


\section{Synthesis of ethyl 4-[hydroxy(phenyl)methyl]-3-nitro-benzoate (9f)}

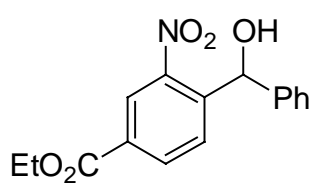

Prepared according to TP 3 from ethyl 4-iodo-3-nitrobenzoate (1f) (642 mg, $2.00 \mathrm{mmol}$ ), $\mathrm{PhMgCl}$ (1.1 mL, $2.2 \mathrm{mmol}, 2.0 \mathrm{M}$ in THF) and benzaldehyde (254 mg, $2.40 \mathrm{mmol})$. Reaction time: $0.5 \mathrm{~h}$. Purification by flash chromatography (pentane $/ \mathrm{CH}_{2} \mathrm{Cl}_{2}=4: 1$ ) yielded title compound 9f (564 mg, 94\%) as a pale yellow oil.

${ }^{1} \mathbf{H}$-NMR $\left(300 \mathrm{MHz}, \mathrm{CDCl}_{3}, 25{ }^{\circ} \mathrm{C}\right): \delta=8.45 \quad\left(\mathrm{~d},{ }^{4} \mathrm{~J}(\mathrm{H}, \mathrm{H})=1.8 \mathrm{~Hz}, 1 \mathrm{H}\right), 8.19(\mathrm{dd}$, $\left.{ }^{3} J(\mathrm{H}, \mathrm{H})=8.4 \mathrm{~Hz},{ }^{4} J(\mathrm{H}, \mathrm{H})=1.8 \mathrm{~Hz}, 1 \mathrm{H}\right), 7.84\left(\mathrm{~d},{ }^{3} J(\mathrm{H}, \mathrm{H})=8.4 \mathrm{~Hz} 1 \mathrm{H}\right), 7.25-7.18(\mathrm{~m}, 5 \mathrm{H})$, $6.40\left(\mathrm{~d},{ }^{3} J(\mathrm{H}, \mathrm{H})=3.5 \mathrm{~Hz}, 1 \mathrm{H}\right), 4.34\left(\mathrm{q},{ }^{3} J(\mathrm{H}, \mathrm{H})=7.1 \mathrm{~Hz}, 2 \mathrm{H}\right), 2.85\left(\mathrm{~d},{ }^{3} J(\mathrm{H}, \mathrm{H})=3.5 \mathrm{~Hz}\right.$, $1 \mathrm{OH}), 1.33\left(\mathrm{t},{ }^{3} \mathrm{~J}(\mathrm{H}, \mathrm{H})=7.1 \mathrm{~Hz}, 3 \mathrm{H}\right) .{ }^{13} \mathrm{C}$-NMR $\left(75 \mathrm{MHz}, \mathrm{CDCl}_{3}, 25^{\circ} \mathrm{C}\right): \delta=164.7,148.5$, 143.1, 141.4, 134.2, 131.4, 129.8, 129.1, 128.8, 127.4, 126.1, 71.8, 62.3, 14.6. MS $(70 \mathrm{eV}$, $\mathrm{EI}), m / z$ (\%): 283 (14) [M-H $\mathrm{H}_{2}^{+}, 266$ (26), 256 (16), 238 (41), 222 (20), 211 (14), 194 (100), 180 (23), 167 (72), 152 (69), 139 (8), 127 (5), 115 (6), 105 (41), 77 (54), 65 (4), 51 (9). IR (KBr): $\tilde{v}=3478(\mathrm{~s}), 2984(\mathrm{~m}), 1728$ (vs), 1620 (s), 1538 (vs), 1493 (s), 1454 (s), 1355 (s), 1288 (s), 1152 (s), 1021 (s), 919 (m), 838 (m), 764 (vs), 749 (vs), 701 (vs), 665 (w), 557 (w). HRMS for $\mathbf{C}_{\mathbf{1 6}} \mathbf{H}_{\mathbf{1 5}} \mathbf{N O}_{\mathbf{5}}$ (301.0950): found: $302.1031[\mathrm{M}+\mathrm{H}]^{+}$.

\section{Synthesis of ethyl 4-(1-hydroxyhexyl)-3-nitrobenzoate (9g)}<smiles>CCCCCC(O)c1ccc(C(=O)OCC)cc1[N+](=O)[O-]</smiles>

Prepared according to TP 3 from ethyl 4-iodo-3-nitrobenzoate (1f) (483 mg, $1.50 \mathrm{mmol}$ ), $\mathrm{PhMgCl}(0.85 \mathrm{~mL}, 1.6 \mathrm{mmol}, 1.9 \mathrm{M}$ in THF) and hexanal (201 mg, $2.00 \mathrm{mmol})$. Reaction time: $2 \mathrm{~h}$. Purification by flash chromatography (pentane/diethyl ether $=5: 1$ ) yielded title compound $9 \mathrm{~g}$ (383 $\mathrm{mg}, 87 \%$ ) as a colourless oil.

${ }^{1} \mathbf{H}$-NMR $\left(300 \mathrm{MHz}, \mathrm{CDCl}_{3}, 25{ }^{\circ} \mathrm{C}\right): \delta=8.43\left(\mathrm{~d}, \quad{ }^{4} J(\mathrm{H}, \mathrm{H})=1.8 \mathrm{~Hz}, 1 \mathrm{H}\right), 8.18(\mathrm{dd}$, $\left.{ }^{3} J(\mathrm{H}, \mathrm{H})=8.4 \mathrm{~Hz},{ }^{4} J(\mathrm{H}, \mathrm{H})=1.8 \mathrm{~Hz}, 1 \mathrm{H}\right), 7.82\left(\mathrm{~d},{ }^{3} J(\mathrm{H}, \mathrm{H})=8.4 \mathrm{~Hz}, 1 \mathrm{H}\right), 5.21-5.17(\mathrm{~m}, 1 \mathrm{H})$, $4.36\left(\mathrm{q},{ }^{3} J(\mathrm{H}, \mathrm{H})=7.1 \mathrm{~Hz}, 2 \mathrm{H}\right), 2.41\left(\mathrm{~s} \_b r, 1 \mathrm{OH}\right), 1.85-1.29(\mathrm{~m}, 8 \mathrm{H}), 0.92-0.85(\mathrm{~m}, 3 \mathrm{H})$. ${ }^{13} \mathrm{C}-\mathrm{NMR}\left(75 \mathrm{MHz}, \mathrm{CDCl}_{3}, 25^{\circ} \mathrm{C}\right): \delta=164.5,147.6,144.9,133.8,130.5,128.4,125.4,69.3$, 61.8, 38.3, 32.7, 25.6, 22.9, 14.2, 14.0. MS (70 eV, EI), m/z (\%): 295 (4) [M+], 278 (9), 250 (12), 224 (100), 207 (26), 176 (58), 162 (14), 149 (17), 121 (11), 105 (14), 77 (7), 65 (13), 43 (31). IR (KBr): $\tilde{v}=3440$ (m), 2957 (s), 1727 (vs), 1620 (m), 1534 (vs), 1466 (m), 1354 (s), 1286 (vs), 1113 (s), 1020 (m), 920 (w), 862 (w), 772 (m), 759 (m), 717 (w). HRMS for $\mathbf{C}_{15} \mathbf{H}_{21} \mathbf{N O}_{5}$ (295.1420): found: $296.1490[\mathrm{M}+\mathrm{H}]^{+}$. 


\section{Synthesis of [hydroxy(phenyl)methyl]-4-nitrobenzonitrile (9h)}<smiles>N#Cc1ccc(C(O)c2ccccc2)c([N+](=O)[O-])c1</smiles>

Prepared according to TP 3 from 4-iodo-3-nitrobenzonitrile (1g) (274 mg, $1.00 \mathrm{mmol}$ ), $\mathrm{PhMgCl}(0.55 \mathrm{~mL}, 1.1 \mathrm{mmol}, 2.0 \mathrm{M}$ in THF) and benzaldehyde (128 mg, $1.20 \mathrm{mmol})$. Reaction time: $0.5 \mathrm{~h}$. Purification by flash chromatography $\left(\mathrm{CH}_{2} \mathrm{Cl}_{2}\right)$ yielded compound $9 \mathrm{~h}$ (240 mg, 94\%) as a yellow oil.

${ }^{1}$ H-NMR $\left(300 \mathrm{MHz}, \quad \mathrm{CDCl}_{3}, \quad 25{ }^{\circ} \mathrm{C}\right): \delta=8.10 \quad\left(\mathrm{~d}, \quad{ }^{4} J(\mathrm{H}, \mathrm{H})=1.8 \mathrm{~Hz}, 1 \mathrm{H}\right), \quad 7.87 \quad(\mathrm{~d}$, $\left.{ }^{3} J(\mathrm{H}, \mathrm{H})=8.4 \mathrm{~Hz}, 1 \mathrm{H}\right), 7.66\left(\mathrm{dd},{ }^{3} J(\mathrm{H}, \mathrm{H})=8.4 \mathrm{~Hz},{ }^{4} J(\mathrm{H}, \mathrm{H})=1.8 \mathrm{~Hz}, 1 \mathrm{H}\right), 7.31-7.18(\mathrm{~m}$, $5 \mathrm{H}), 6.36\left(\mathrm{~d},{ }^{3} J(\mathrm{H}, \mathrm{H})=3.6 \mathrm{~Hz}, 1 \mathrm{H}\right), 2.7\left(\mathrm{~d},{ }^{3} J(\mathrm{H}, \mathrm{H})=3.6 \mathrm{~Hz}, 1 \mathrm{OH}\right) .{ }^{13} \mathbf{C}-\mathbf{N M R}(75 \mathrm{MHz}$, $\left.\mathrm{CDCl}_{3}, 25^{\circ} \mathrm{C}\right): \delta=149.1,139.4,138.8,132.1,131.0,128.6,128.0,127.5,126.1,124.2$, 116.0, 69.9. MS (70 eV, EI), m/z (\%): 236 (11) [M- $\left.\mathrm{H}_{2} \mathrm{O}\right]^{+}, 219$ (73), 206 (25), 192 (100), 177 (23), 165 (15), 151 (27), 129 (7), 105 (20), 77 (59), 63 (5), 51 (11). IR (KBr): $\tilde{v}=3468$ (br, s), 3085 (s), 2238 (s), 1673 (w), 1601 (m), 1584 (vs), 1454 (s), 1350 (vs), 1299 (s), 1146 (m), 1077 (m), 1023 (s), 917 (w), 846 (vs), 759 (s), 701 (vs), 592 (w), 554 (w). HRMS for $\mathbf{C}_{14} \mathbf{H}_{10} \mathbf{N}_{\mathbf{2}} \mathbf{O}_{3}$ (254.0691): found: $252.0546[\mathrm{M}-2 \mathrm{H}]^{+}$.

\section{Synthesis of 1-(4-cyano-2-nitrophenyl)-1-hexanol (9i)}

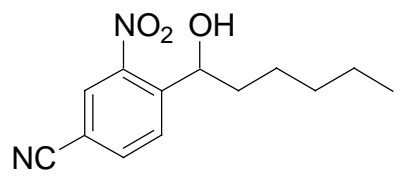

Prepared according to TP 3 from 3-iodo-4-nitrobenzonitrile (1g) (411 mg, $1.50 \mathrm{mmol}$ ), $\mathrm{PhMgCl}(0.85 \mathrm{~mL}, 1.6 \mathrm{mmol}, 1.9 \mathrm{M}$ in THF) and hexanal $(225 \mathrm{mg}, 2.30 \mathrm{mmol})$. Reaction time: $2 \mathrm{~h}$. Purification by flash chromatography (pentane/diethyl ether $=4: 1$ ) yielded title compound $9 \mathbf{i}$ (234 $\mathrm{mg}, 63 \%)$ as a pale yellow oil.

${ }^{1} \mathbf{H}-\mathbf{N M R} \quad\left(300 \mathrm{MHz}, \quad \mathrm{CDCl}_{3}, \quad 25{ }^{\circ} \mathrm{C}\right): \delta=8.17 \quad\left(\mathrm{~d}, \quad{ }^{4} J(\mathrm{H}, \mathrm{H})=1.8 \mathrm{~Hz}, \quad 1 \mathrm{H}\right), 8.00 \quad(\mathrm{~d}$, $\left.{ }^{3} J(\mathrm{H}, \mathrm{H})=8.4 \mathrm{~Hz}, 1 \mathrm{H}\right), 7.87\left(\mathrm{dd},{ }^{3} J(\mathrm{H}, \mathrm{H})=8.4 \mathrm{~Hz},{ }^{4} J(\mathrm{H}, \mathrm{H})=1.8 \mathrm{~Hz}, 1 \mathrm{H}\right), 5.33-5.29(\mathrm{~m}$, $1 \mathrm{H}), 2.47$ (s_br, 1H), 1.81-1.25 (m, $8 \mathrm{H}), 1.33-1.26$ (m, $3 \mathrm{H}) .{ }^{13} \mathbf{C}-\mathbf{N M R}\left(75 \mathrm{MHz}, \mathrm{CDCl}_{3}\right.$, $\left.25^{\circ} \mathrm{C}\right): \delta=149.3,147.7,137.9,131.4,129.8,118.3,113.9,70.9,40.3,33.1,27.4,24.3,15.7$. MS (70 eV, EI), m/z (\%) 249 (1) [M+H] $]^{+}, 177$ (100), 160 (51), 145 (9), 129 (27), 117 (11), 104 (11), 77 (3). IR (KBr): $\widetilde{v}=3438$ (m), 2956 (m), 2930 (m), 2238 (s), 1617 (w), 1537 (vs), 1466 (m), 1356 (s), 1055 (m), 849 (m), 818 (w). HRMS for $\mathbf{C}_{\mathbf{1 3}} \mathbf{H}_{\mathbf{1 6}} \mathbf{N}_{\mathbf{2}} \mathbf{O}_{\mathbf{3}}$ (248.1161): found: $249.1262[\mathrm{M}+\mathrm{H}]^{+}$. 


\section{Synthesis of [2-nitro-4-(1-piperidinylcarbonyl)phenyl](phenyl)methanol (9j)}

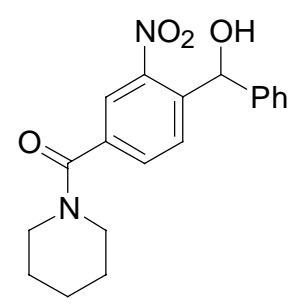

Prepared according to TP 3 from 1-(4-iodo-3-nitrobenzoyl)piperidine (1h) (360 mg, $1.00 \mathrm{mmol}), \mathrm{PhMgCl}(0.63 \mathrm{~mL}, 1.1 \mathrm{mmol}, 1.8 \mathrm{M}$ in THF) and benzaldehyde $(212 \mathrm{mg}$, $2.00 \mathrm{mmol}$ ). Reaction time: $1 \mathrm{~h}$. Purification by flash chromatography (pentane/diethyl ether $=2: 1)$ yielded title compound $\mathbf{9 j}$ as a colourless oil $(285 \mathrm{mg}, 84 \%)$.

${ }^{1} \mathrm{H}-\mathrm{NMR} \quad\left(300 \mathrm{MHz}, \quad \mathrm{CDCl}_{3}, \quad 25{ }^{\circ} \mathrm{C}\right): \delta=7.89\left(\mathrm{~d},{ }^{4} J(\mathrm{H}, \mathrm{H})=1.8 \mathrm{~Hz}, 1 \mathrm{H}\right), \quad 7.81 \quad(\mathrm{~d}$, $\left.{ }^{3} J(\mathrm{H}, \mathrm{H})=8.4 \mathrm{~Hz}, 1 \mathrm{H}\right), 7.58\left(\mathrm{dd},{ }^{3} J(\mathrm{H}, \mathrm{H})=8.4 \mathrm{~Hz},{ }^{4} J(\mathrm{H}, \mathrm{H})=1.8 \mathrm{~Hz}, 1 \mathrm{H}\right), 7.26-7.18(\mathrm{~m}$, $5 \mathrm{H}), 6.41\left(\mathrm{~d},{ }^{2} J(\mathrm{H}, \mathrm{H})=3.8 \mathrm{~Hz}, 1 \mathrm{H}\right), 3.68\left(\mathrm{~s} \_b r, 2 \mathrm{H}\right), 3.50\left(\mathrm{~d},{ }^{2} J(\mathrm{H}, \mathrm{H})=3.8 \mathrm{~Hz}, 1 \mathrm{OH}\right), 3.31$ (s_br, $2 \mathrm{H}), 1.70-1.45(\mathrm{~m}, 6 \mathrm{H}) .{ }^{13} \mathrm{C}-\mathrm{NMR}\left(75 \mathrm{MHz}, \mathrm{CDCl}_{3}, 25{ }^{\circ} \mathrm{C}\right): \delta=167.5,147.9,141.4$, 140.0, 136.5, 131.5, 129.6, 128.6, 128.1, 127.0, 123.2, 70.9, 48.9, 43.4, 26.5, 25.5, 24.4. MS (70 eV, EI), m/z (\%): 337 (100) [M-2H] , 321 (23), 311 (9), 290 (24), 254 (54), 224 (10), 207 (9), 197 (13), 180 (43), 152 (40), 105 (23), 77 (17). IR (KBr): $\widetilde{v}=3392$ (m), 2939 (w), 1616 (vs), 1532 (vs), 1495 (m), 1445 (m), 1353 (m), 1287 (m), 1180 (w), 1117 (s), 1041 (w), 1026 (m), 1010 (w), 906 (w), 854 (w), 752 (m), 700 (m). HRMS for $\mathbf{C}_{\mathbf{1 9}} \mathbf{H}_{\mathbf{2 0}} \mathbf{N}_{\mathbf{2}} \mathbf{O}_{\mathbf{4}}$ (340.1423): found: $338.1263[\mathrm{M}-2 \mathrm{H}]^{+} . \mathbf{C}_{\mathbf{1 9}} \mathbf{H}_{\mathbf{2 0}} \mathbf{N}_{\mathbf{2}} \mathbf{O}_{\mathbf{4}}$ : required: $\mathrm{C}$ : 67.05; $\mathrm{H}: 5.92 ; \mathrm{N}: 8.23$; found: $\mathrm{C}$ : $66.73 ; \mathrm{H}: 5.89 ; \mathrm{N}: 8.18$.

\section{Synthesis of (3,5-dimethyl-2-nitrophenyl)(phenyl)methanol (9k)}

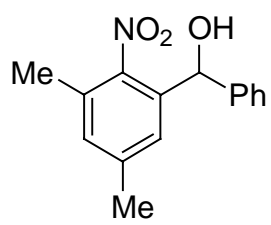

Prepared according to TP 3 from 2-iodo-4,6-dimethyl-nitrobenzene (1k) (277 mg, $1.00 \mathrm{mmol}), \mathrm{PhMgCl}(0.63 \mathrm{~mL}, 1.1 \mathrm{mmol}, 1.75 \mathrm{M}$ in THF) and benzaldehyde $(212 \mathrm{mg}$, $2.00 \mathrm{mmol}$ ). Reaction time: $2 \mathrm{~h}$. Purification by flash chromatography (pentane/diethyl ether $=29: 1)$ yielded benzylic alcohol $9 \mathbf{k}$ as a colourless oil $(189 \mathrm{mg}, 74 \%)$.

${ }^{1}$ H-NMR $\left(400 \mathrm{MHz}, \mathrm{CDCl}_{3}, 25{ }^{\circ} \mathrm{C}\right): \delta=7.34-7.28$ (m, $\left.5 \mathrm{H}\right), 7.14$ (s_br, $\left.1 \mathrm{H}\right), 7.02$ (s_br, $1 \mathrm{H}), 5.92(\mathrm{~s}, 1 \mathrm{H}), 2.31(\mathrm{~s}, 3 \mathrm{H}), 2.28(\mathrm{~s}, 3 \mathrm{H}),{ }^{13} \mathrm{C}-\mathrm{NMR}\left(100 \mathrm{MHz}, \mathrm{CDCl}_{3}, 25{ }^{\circ} \mathrm{C}\right)$ : $\delta=147.8,141.5,141.0,135.5,131.4,129.9,128.5,127.9,126.7,126.5,71.4,21.3,17.6$. MS (70 eV, EI), m/z (\%): 257 (not detectable), 239 (12) $\left[\mathrm{M}-\mathrm{H}_{2} \mathrm{O}\right]^{+}, 222$ (23), 194 (100), 180 (79), 165 (36), 105 (20), 77 (37). IR (KBr): $\tilde{v}=3411$ (w), 1599 (w), 1524 (vs), 1495 (m), 1451 
(w), 1365 (s), 1175 (w), 1051 (m), 842 (m), 759 (w), 701 (s). $\mathbf{C}_{\mathbf{1 5}} \mathbf{H}_{\mathbf{1 5}} \mathbf{N O}_{\mathbf{3}}$ : required: C: 70.02 ; H: 5.88; N: 5.44; found: C: 70.33; H: 5.76; N: 5.24 .

\section{Synthesis of (4-iodo-2-nitrophenyl)(phenyl)methanol (9l)}

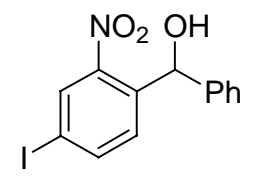

Prepared according to TP 3 from 2,5-diiodo-nitrobenzene (1m) (563 mg, $1.50 \mathrm{mmol}$ ), $\mathrm{PhMgCl}(0.80 \mathrm{~mL}, 1.6 \mathrm{mmol}, 2.0 \mathrm{M}$ in THF) and benzaldehyde (190 mg, $1.80 \mathrm{mmol})$. Reaction time: $0.5 \mathrm{~h}$. Purification by flash chromatography (pentane/diethyl ether $=4: 1$ ) yielded title compound 91 (458 $\mathrm{mg}, 86 \%)$ as a yellow oil.

${ }^{1} \mathbf{H}$-NMR $\left(300 \mathrm{MHz}, \mathrm{CDCl}_{3}, 25^{\circ} \mathrm{C}\right): \delta=8.15 \quad\left(\mathrm{~d},{ }^{4} J(\mathrm{H}, \mathrm{H})=1.8 \mathrm{~Hz}, 1 \mathrm{H}\right), 7.86$ (dd, $\left.{ }^{3} J(\mathrm{H}, \mathrm{H})=8.4 \mathrm{~Hz},{ }^{4} J(\mathrm{H}, \mathrm{H})=1.8 \mathrm{~Hz}, 1 \mathrm{H}\right), 7.42\left(\mathrm{~d},{ }^{3} J(\mathrm{H}, \mathrm{H})=8.4 \mathrm{~Hz}, 1 \mathrm{H}\right), 7.28-7.18(\mathrm{~m}, 5 \mathrm{H})$, 6.30 (s, $1 \mathrm{H}), 2,45$ (s_br, $1 \mathrm{H}) .{ }^{13} \mathrm{C}-\mathbf{N M R}\left(75 \mathrm{MHz}, \mathrm{CDCl}_{3}, 25^{\circ} \mathrm{C}\right): \delta=141.5,141.2,140.0$, 137.1, 132.1, 129.8, 127.7, 127.2, 125.9, 91.2, 70.2. MS (70 eV, EI), $m / z(\%): 353(3)\left[\mathrm{M}^{+}\right]$, 337 (18), 320 (82), 308 (6), 293 (38), 260 (8), 231 (13), 182(22), 166 (100), 152 (73), 139 (25), 109 (22), 77 (58), 63 (10), 51 (14). IR (KBr): $\tilde{v}=3369$ (br), 1530 (vs), 1469 (s), 1347 (vs), 1275 (m), 1179 (m), 1034 (s), 871 (m), 808 (m), 763 (s), 700 (s). HRMS for $\mathbf{C}_{13} \mathbf{H}_{10} \mathrm{INO}_{3}$ (354.9705): found: $352.9544[\mathrm{M}-2 \mathrm{H}]^{+}$.

\section{Synthesis of 1-(4-iodo-2-nitrophenyl)-1-hexanol (9m)}

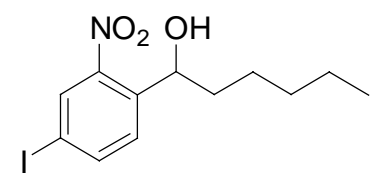

Prepared according to TP 3 from 2,5-diiodonitrobenzol (1m) (562 mg, $1.50 \mathrm{mmol}$ ), PhMgCl (0.80 mL, $1.6 \mathrm{mmol}, 2.0 \mathrm{M}$ in THF) and hexanealdehyde (201 mg, $2.00 \mathrm{mmol})$. Reaction time: $2 \mathrm{~h}$. Purification by flash chromatography (pentane/diethyl ether $=9: 1$ ) yielded title compound 9m (480 mg, 86\%) as a pale yellow oil.

${ }^{1} \mathbf{H}$-NMR $\left(300 \mathrm{MHz}, \mathrm{CDCl}_{3}, 25^{\circ} \mathrm{C}\right): \delta=8.21 \quad\left(\mathrm{~d},{ }^{4} J(\mathrm{H}, \mathrm{H})=1.8 \mathrm{~Hz}, 1 \mathrm{H}\right), 7.96(\mathrm{dd}$, $\left.{ }^{3} J(\mathrm{H}, \mathrm{H})=8.4 \mathrm{~Hz},{ }^{4} J(\mathrm{H}, \mathrm{H})=1.8 \mathrm{~Hz}, 1 \mathrm{H}\right), 7.55\left(\mathrm{~d},{ }^{3} J(\mathrm{H}, \mathrm{H})=8.4 \mathrm{~Hz} 1 \mathrm{H}\right), 5.22-5.18(\mathrm{~m}, 1 \mathrm{H})$, 2.47 (s_br, $1 \mathrm{OH}), 1.83-1.27(\mathrm{~m}, 8 \mathrm{H}), 0.92-0.88(\mathrm{~m}, 3 \mathrm{H}) .{ }^{13} \mathrm{C}-\mathrm{NMR}\left(75 \mathrm{MHz}, \mathrm{CDCl}_{3}\right.$, $\left.25^{\circ} \mathrm{C}\right): \delta=148.1,142.3,140.0,132.7,129.8,91.4,69.1,38.1,31.4,25.7,22.5,14.0 . \mathrm{MS}$ (70 eV, EI), $m / z(\%): 349$ (not detectable), $332(2)[\mathrm{M}-\mathrm{OH}]^{+}, 278$ (100), 261 (16), 229 (24), 203 (4), 76 (3). IR (KBr): $\tilde{v}=3418$ (w), 2928 (m), 1529 (vs), 1466 (m), 1347 (vs), 1054 (m), 869 (w), 832 (w), 761 (w). HRMS for $\mathbf{C}_{\mathbf{1 2}} \mathbf{H}_{\mathbf{1 6}} \mathbf{I N O} \mathbf{O}_{\mathbf{3}}\left(332.0148\right.$ [M-OH] $\left.{ }^{+}\right)$: found: 332.0169 $[\mathrm{M}-\mathrm{OH}]^{+} . \mathbf{C}_{\mathbf{1 2}} \mathbf{H}_{\mathbf{1 6}} \mathbf{I N O}_{3}$ : required: C: $41.28 ; \mathrm{H}: 4.62 ; \mathrm{I}: 36.35 ; \mathrm{N}: 4.01$; found: $\mathrm{C}: 41.69, \mathrm{H}$ : 4.32 ; I: $36.76 ; \mathrm{N}: 4.00$. 


\section{Synthesis of \{3-[hydroxy(phenyl)methyl]-4-nitrophenyl\}(phenyl)methanone (9n)}

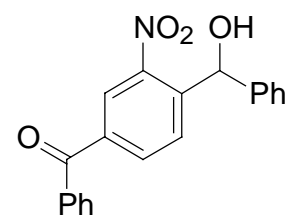

Prepared according to TP 3 from 4-iodo-3-nitrobenzophenone (1o) (353 mg, $1.00 \mathrm{mmol}$ ), $\mathrm{PhMgCl}(0.63 \mathrm{~mL}, 1.1 \mathrm{mmol}, 1.75 \mathrm{M}$ in $\mathrm{THF})$ and benzaldehyde $(212 \mathrm{mg}, 2.00 \mathrm{mmol})$. Reaction time: $1 \mathrm{~h}$. Purification by flash chromatography (pentane/diethyl ether $=4: 1$ ) yielded ketone $9 \mathbf{n}$ as a yellow oil (309 mg, 93\%).

${ }^{1}$ H-NMR $\left(400 \mathrm{MHz}, \mathrm{CDCl}_{3}, 25{ }^{\circ} \mathrm{C}\right): \delta=8.29\left(\mathrm{~d},{ }^{4} J(\mathrm{H}, \mathrm{H})=1.7 \mathrm{~Hz}, 1 \mathrm{H}\right), 8.04$ (dd, $\left.{ }^{3} J(\mathrm{H}, \mathrm{H})=8.2 \mathrm{~Hz},{ }^{4} J(\mathrm{H}, \mathrm{H})=1.7 \mathrm{~Hz}, 1 \mathrm{H}\right), 7.96\left(\mathrm{~d},{ }^{3} J(\mathrm{H}, \mathrm{H})=8.2 \mathrm{~Hz}, 1 \mathrm{H}\right), 7.79-7.76(\mathrm{~m}, 2 \mathrm{H})$, $7.63\left(\mathrm{dt},{ }^{3} J(\mathrm{H}, \mathrm{H})=7.5 \mathrm{~Hz},{ }^{4} J(\mathrm{H}, \mathrm{H})=1.4 \mathrm{~Hz}, 1 \mathrm{H}\right), 7.54-7.48(\mathrm{~m}, 2 \mathrm{H}), 7.35-7.31(\mathrm{~m}, 5 \mathrm{H})$, $6.51(\mathrm{~s}, 1 \mathrm{H}) .{ }^{13} \mathrm{C}-\mathbf{N M R}\left(100 \mathrm{MHz}, \mathrm{CDCl}_{3}, 25{ }^{\circ} \mathrm{C}\right): \delta=193.8,148.0,142.2,141.0,137.8$, 136.3, 133.9, 133.3, 129.9, 129.4, 128.8, 128.7, 128.4, 127.1, 125.9, 70.4. MS (70 eV, EI), $m / z(\%): 331(0.5)[\mathrm{M}-2 \mathrm{H}]^{+}, 315$ (15), 300 (38), 270 (6), 238 (24), 224 (9), 194 (56), 166 (11), 152 (11), 105 (100), 77 (45). IR (KBr): $\tilde{v}=3437$ (m), 3063 (w), 1661 (vs), 1615 (s), 1598 (s), 1577 (m), 1532 (vs), 1493 (m), 1448 (s), 1350 (vs), 1319 (vs), 1281 (vs), 1180 (s), 1074 (m), $1037(\mathrm{~m}), 1024(\mathrm{~m}), 978(\mathrm{~m}), 878$ )w), $758(\mathrm{~m}), 718$ (s), 699 (s), 676 (s). HRMS for $\mathbf{C}_{20} \mathbf{H}_{15} \mathbf{N O}_{4}$ (333.1001): found: $331.0826[\mathrm{M}-2 \mathrm{H}]^{+}$.

\section{Synthesis of compounds 12}

\section{Synthesis of (3-nitro-2-pyridinyl)(phenyl)methanol (12a)}

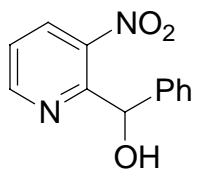

Prepared according to TP 3 from 2-iodo-3-nitropyridine (5a) (125 mg, $0.50 \mathrm{mmol}$ ), $\mathrm{PhMgCl}$ (0.35 mL, $0.55 \mathrm{mmol}, 1.5 \mathrm{M}$ in THF) and benzaldehyde $(106 \mathrm{mg}, 1.00 \mathrm{mmol})$ at $-78{ }^{\circ} \mathrm{C}$. Reaction time: $2 \mathrm{~h}$. Purification by flash chromatography (pentane/diethyl ether $=1: 1$ ) yielded benzylic alcohol 12a as a pale yellow oil (64 $\mathrm{mg}, 55 \%)$.

${ }^{1}$ H-NMR $\left(400 \mathrm{MHz}, \mathrm{CDCl}_{3}, 25{ }^{\circ} \mathrm{C}\right): \delta=8.90\left(\mathrm{dd},{ }^{3} J(\mathrm{H}, \mathrm{H})=4.9 \mathrm{~Hz},{ }^{4} J(\mathrm{H}, \mathrm{H})=1.7 \mathrm{~Hz}, 1 \mathrm{H}\right)$, $8.36\left(\mathrm{dd}, \quad{ }^{3} J(\mathrm{H}, \mathrm{H})=8.4 \mathrm{~Hz}, \quad{ }^{4} J(\mathrm{H}, \mathrm{H})=1.7 \mathrm{~Hz}, \quad 1 \mathrm{H}\right), \quad 7.50 \quad\left(\mathrm{dd}, \quad{ }^{3} J(\mathrm{H}, \mathrm{H})=8.1 \mathrm{~Hz}\right.$, $\left.{ }^{3} J(\mathrm{H}, \mathrm{H})=4.9 \mathrm{~Hz}, 1 \mathrm{H}\right), 7.31-7.21(\mathrm{~m}, 5 \mathrm{H}), 6.50\left(\mathrm{~d},{ }^{3} J(\mathrm{H}, \mathrm{H})=6.6 \mathrm{~Hz}, 1 \mathrm{H}\right), 5.38(\mathrm{~d}$, $\left.{ }^{3} J(\mathrm{H}, \mathrm{H})=6.6 \mathrm{~Hz}, 1 \mathrm{H}\right) .{ }^{13} \mathrm{C}$-NMR $\left(100 \mathrm{MHz}, \mathrm{CDCl}_{3}, 25{ }^{\circ} \mathrm{C}\right): \delta=156.3,152.3,144.2,141.7$, 134.0, 129.0, 128.5, 128.0, 123.8, 72.5. MS (70 eV, EI), m/z (\%): 230 (5) [M $\left.\mathrm{M}^{+}\right], 213$ (15), 196 (43), 182 (60) 168 (91), 155 (87), 137 (44), 127 (29), 105 (66), 94 (9), 77 (100), 63 (7). IR $(\mathrm{KBr}): \tilde{v}=3396(\mathrm{w}), 1597(\mathrm{~m}), 1567(\mathrm{~m}), 1529(\mathrm{vs}), 1453(\mathrm{~m}), 1349$ (vs), 1188 (m), 1041 
(m), 835 (m), 767 (m), 745 (s), 700 (s), 606 (s). HRMS for $\mathbf{C}_{\mathbf{1 2}} \mathbf{H}_{\mathbf{1 0}} \mathbf{N}_{\mathbf{2}} \mathbf{O}_{\mathbf{3}}$ (230.0691): found: 230.0693 .

\section{Synthesis of (6-nitro-5-quinolinyl)(phenyl)methanol (12b)}

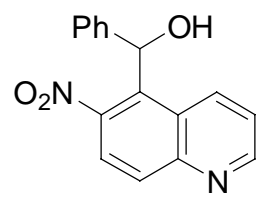

Prepared according to TP 3 from 5-iodo-6-nitroquinoline (5b) (300 mg, $1.00 \mathrm{mmol}$ ), $\mathrm{PhMgCl}$ (0.63 mL, $1.1 \mathrm{mmol}, 1.75 \mathrm{M}$ in THF) and benzaldehyde (212 mg, $2.00 \mathrm{mmol})$. Reaction time: $1 \mathrm{~h}$. Purification by flash chromatography (pentane/diethyl ether $=1: 1$ ) yielded quinoline derivative $\mathbf{1 2 b}$ as a pale yellow solid $(218 \mathrm{mg}, 78 \%)$.

mp.: $\quad 138.2-139.5^{\circ} \mathrm{C} .{ }^{1} \mathbf{H}-\mathrm{NMR}\left(600 \mathrm{MHz}, \mathrm{CDCl}_{3}, 25^{\circ} \mathrm{C}\right): \delta=8.79\left(\mathrm{dd},{ }^{3} J(\mathrm{H}, \mathrm{H})=4.9 \mathrm{~Hz}\right.$, $\left.{ }^{4} J(\mathrm{H}, \mathrm{H})=1.7 \mathrm{~Hz}, 1 \mathrm{H}\right), 8.64\left(\mathrm{~d},{ }^{3} J(\mathrm{H}, \mathrm{H})=8.1 \mathrm{~Hz}, 1 \mathrm{H}\right), 8.04\left(\mathrm{~d},{ }^{3} J(\mathrm{H}, \mathrm{H})=9.0 \mathrm{~Hz}, 1 \mathrm{H}\right), 7.84$ $\left(\mathrm{d},{ }^{3} J(\mathrm{H}, \mathrm{H})=9.0 \mathrm{~Hz}, 1 \mathrm{H}\right), 7.29\left(\mathrm{~d},{ }^{3} J(\mathrm{H}, \mathrm{H})=7.3 \mathrm{~Hz}, 2 \mathrm{H}\right), 7.17-7.14(\mathrm{~m}, 2 \mathrm{H}), 7.08(\mathrm{t}$, $\left.{ }^{3} J(\mathrm{H}, \mathrm{H})=7.3 \mathrm{~Hz}, 1 \mathrm{H}\right), 6.42(\mathrm{~s}, 1 \mathrm{H}), 6.11\left(\mathrm{~s} \_b r, 1 \mathrm{OH}\right), 5.38\left(\mathrm{~d},{ }^{3} J(\mathrm{H}, \mathrm{H})=6.6 \mathrm{~Hz}, 1 \mathrm{H}\right) .{ }^{13} \mathrm{C}-$ NMR $\left(150 \mathrm{MHz}, \mathrm{CDCl}_{3}, 25{ }^{\circ} \mathrm{C}\right): \delta=151.7,149.0,147.6,142.5,137.3,135.1,130.8,127.9$, 126.7, 126.3, 125.5, 122.5, 121.3, 68.8. MS (70 eV, EI), m/z (\%): $278(0.2)[\mathrm{M}-2 \mathrm{H}]^{+}, 246$ (23), 232 (12), 218 (100), 206 (17), 176 (8), 151 (4), 128 (4), 105 (15), 77 (23). IR (KBr): $\tilde{v}=3272(\mathrm{~m}), 1597(\mathrm{w}), 1530$ (vs), 1499 (s), 1450 (m), 1359 (s), 1260 (w), 1045 (m), 1026 (w), 908 (w), 836 (s), 808 (s), 778 (m), 759 (m), 736 (m), 709 (s), 581(w). HRMS for $\mathbf{C}_{16} \mathbf{H}_{12} \mathbf{N}_{2} \mathbf{O}_{3}$ (280.0848): found: $278.0686[\mathrm{M}-2 \mathrm{H}]^{+}$.

\section{Synthesis of compounds 14}

\section{Synthesis of 1-allyl-2-nitrobenzene (14a)}

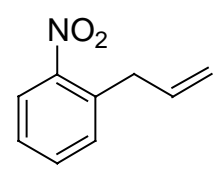

Prepared according to TP 3 from 2-iodo-nitrobenzene (1a) (498 mg, $2.00 \mathrm{mmol}$ ), $\mathrm{PhMgCl}$ (1.3 mL, $2.2 \mathrm{mmol}, 1.7 \mathrm{M}$ in THF), CuCN.2LiCl (2.2 mL, $2.2 \mathrm{mmol}, 1.0 \mathrm{M}$ in THF) and allyl bromide ( $290 \mathrm{mg}, 2.40 \mathrm{mmol}$ ). Reaction time: $45 \mathrm{~min}$. Purification by flash chromatography (pentane $\left./ \mathrm{CH}_{2} \mathrm{Cl}_{2}=4: 1\right)$ furnished title compound $\mathbf{1 4 a}(244 \mathrm{mg}, 75 \%)$ as a red liquid.

${ }^{1} \mathbf{H}-\mathbf{N M R}\left(\mathrm{CDCl}_{3}, 300 \mathrm{MHz}\right): \delta=7.81\left(\mathrm{dd},{ }^{3} J_{\mathrm{H}-\mathrm{H}}=8.0 \mathrm{~Hz},{ }^{4} J_{\mathrm{H}-\mathrm{H}}=1.3 \mathrm{~Hz}, 1 \mathrm{H}\right), 7.45\left(\mathrm{dt},{ }^{3} J_{\mathrm{H}-}\right.$ $\left.\mathrm{H}=8.0 \mathrm{~Hz},{ }^{4} J_{\mathrm{H}-\mathrm{H}}=1.3 \mathrm{~Hz}, 1 \mathrm{H}\right), 7.30-7.25(\mathrm{~m}, 2 \mathrm{H}), 5.95-5.82(\mathrm{~m}, 1 \mathrm{H}), 5.05-4.96(\mathrm{~m}, 2 \mathrm{H})$, $3.60\left(\mathrm{~d},{ }^{3} J_{\mathrm{H}-\mathrm{H}}=8.0 \mathrm{~Hz}, 2 \mathrm{H}\right) .{ }^{13} \mathrm{C}-\mathrm{NMR}\left(\mathrm{CDCl}_{3}, 75 \mathrm{MHz}\right): \delta=149.7,135.4,135.2,133.4$, 132.3, 127.7, 125.0, 117.5, 34.3. MS (70 eV, EI), m/z (\%): $162\left(\mathrm{M}^{+}, 22\right.$ ), 146 (68), 134 (56), 
129 (23), 115 (100), 104 (19), 91 (51), 77 (39), 65 (28), 51 (18). IR (KBr): $\tilde{v} 3081$ (w), 1610 (m), 1526 (vs), 1435 (m), 1349 (s), 1147 (w), 995 (w), 921 (m), 857 (m), 786 (m), 741 (m), 705 (w), 672 (w), 643 (w). HRMS for $\mathbf{C}_{9} \mathbf{H}_{9} \mathbf{N O}_{2}$ (163.0633): found: 163.0642.

\section{Synthesis of ethyl 2-(4-methoxy-2-nitrobenzyl)acrylate (14b)}

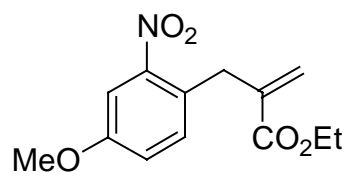

Prepared according to TP 3 from 1-iodo-4-methoxy-2-nitrobenzene (1c) (558 mg, $2.00 \mathrm{mmol}), \mathrm{PhMgCl}(1.3 \mathrm{~mL}, 2.2 \mathrm{mmol}, 1.7 \mathrm{M}$ in THF), CuCN.2LiCl (2.2 mL, $2.2 \mathrm{mmol}$, $1.0 \mathrm{M}$ in THF) and ethyl (2-bromomethyl)acrylate (463 mg, $2.40 \mathrm{mmol})$. Reaction time: 45 min. Purification by flash chromatography (pentane/diethyl ether $=9: 1$ ) furnished title compound 14b (381 mg, 72\%) as an orange liquid.

${ }^{1}$ H-NMR $\left(300 \mathrm{MHz}, \mathrm{CDCl}_{3}, \quad 25^{\circ} \mathrm{C}\right): \delta=7.38 \quad\left(\mathrm{~d}, \quad{ }^{4} J(\mathrm{H}, \mathrm{H})=1.8 \mathrm{~Hz}, 1 \mathrm{H}\right), 7.17 \quad(\mathrm{~d}$, $\left.{ }^{3} J(\mathrm{H}, \mathrm{H})=8.5 \mathrm{~Hz}, 1 \mathrm{H}\right), 7.01\left(\mathrm{dd},{ }^{3} J(\mathrm{H}, \mathrm{H})=8.5 \mathrm{~Hz},{ }^{4} J(\mathrm{H}, \mathrm{H})=1.8 \mathrm{~Hz}, 1 \mathrm{H}\right), 6.17-6.14(\mathrm{~m}$, $1 \mathrm{H}), 5.29-5.27(\mathrm{~m}, 1 \mathrm{H}), 4.12\left(\mathrm{q},{ }^{3} J(\mathrm{H}, \mathrm{H})=7.1 \mathrm{~Hz}, 2 \mathrm{H}\right), 3.82(\mathrm{~s}, 2 \mathrm{H}), 3.77(\mathrm{~s}, 3 \mathrm{H}), 1.18(\mathrm{t}$, $\left.{ }^{3} J(\mathrm{H}, \mathrm{H})=7.1 \mathrm{~Hz}, 3 \mathrm{H}\right) .{ }^{13} \mathrm{C}-\mathrm{NMR}\left(75 \mathrm{MHz}, \mathrm{CDCl}_{3}, 25^{\circ} \mathrm{C}\right): \delta=165.4,157.6,148.8,137.7$, 132.3, 124.4, 118.6, 108.4, 59.9, 54.8, 33.2, 13.1. MS (70 eV, EI), $m / z(\%): 265$ (2) $\left[\mathrm{M}^{+}\right], 248$ (23), 236 (100), 220 (52),202 (20), 190 (60), 174 (63), 164 (37), 146 (56), 132 (28), 103 (32), 91 (24), 77 (42), 63 (15), 51 (18). IR (KBr): $\widetilde{v}=2982$ (m), 1715 (s), 1625 (m), 1531 (vs), 1410 (m), 1352 (s), 1252 (s), 1150 (s), 1034 (s), 945 (w), 814 (m), 757 (w). HRMS for $\mathbf{C}_{13} \mathbf{H}_{15} \mathbf{N O}_{5}$ (265.0950): found: $264.0888[\mathrm{M}-\mathrm{H}]^{+}$.

\section{Synthesis of ethyl 4-[2-(ethoxycarbonyl)-2-propenyl]-3-nitro-benzoate (14c)}<smiles>C=C(COCC)Cc1ccc(C(=O)OCC)cc1[N+](=O)[O-]</smiles>

Prepared according to TP 3 from ethyl 4-iodo-3-nitrobenzoate (1f) (642 mg, $2.00 \mathrm{mmol}$ ), $\mathrm{PhMgCl}$ (1.1 mL, $2.2 \mathrm{mmol}, 2.0 \mathrm{M}$ in THF), CuCN.2LiCl (2.2 mL, $2.2 \mathrm{mmol}, 1.0 \mathrm{M}$ in THF) and ethyl (2-bromomethyl) acrylate $(463 \mathrm{mg}, 2.4 \mathrm{mmol})$. Reaction time: $1 \mathrm{~h}$. Purification by flash chromatography (pentane/diethyl ether $=85: 15$ ) yielded the title compound $\mathbf{1 4 c}$ (461 mg, 75\%) as a yellow oil.

${ }^{1} \mathbf{H}-\mathbf{N M R}\left(300 \mathrm{MHz}, \mathrm{CDCl}_{3}, 25^{\circ} \mathrm{C}\right): \delta=8.47 \quad\left(\mathrm{~d},{ }^{4} J(\mathrm{H}, \mathrm{H})=1.8 \mathrm{~Hz}, 1 \mathrm{H}\right), 8.10 \quad(\mathrm{dd}$, $\left.{ }^{3} J(\mathrm{H}, \mathrm{H})=8.4 \mathrm{~Hz}, \quad{ }^{4} J(\mathrm{H}, \mathrm{H})=1.8 \mathrm{~Hz}, \quad 1 \mathrm{H}\right), 7.39 \quad\left(\mathrm{~d},{ }^{3} J(\mathrm{H}, \mathrm{H})=8.4 \mathrm{~Hz} 1 \mathrm{H}\right), 6.24 \quad(\mathrm{~d}$, $\left.{ }^{2} J(\mathrm{H}, \mathrm{H})=0.9 \mathrm{~Hz}, 1 \mathrm{H}\right), 5.40\left(\mathrm{~d},{ }^{2} J(\mathrm{H}, \mathrm{H})=0.9 \mathrm{~Hz}, 1 \mathrm{H}\right), 4.34\left(\mathrm{q},{ }^{3} J(\mathrm{H}, \mathrm{H})=7.1 \mathrm{~Hz}, 2 \mathrm{H}\right), 4.09$ $\left(\mathrm{q},{ }^{3} J(\mathrm{H}, \mathrm{H})=7.1 \mathrm{~Hz}, 2 \mathrm{H}\right), 3.95(\mathrm{~s}, 2 \mathrm{H}), 1.34\left(\mathrm{t},{ }^{3} J(\mathrm{H}, \mathrm{H})=7.1 \mathrm{~Hz}, 3 \mathrm{H}\right), 1.17 \quad(\mathrm{t}$, $\left.{ }^{3} J(\mathrm{H}, \mathrm{H})=7.1 \mathrm{~Hz}, 3 \mathrm{H}\right) .{ }^{13} \mathrm{C}-\mathrm{NMR}\left(75 \mathrm{MHz}, \mathrm{CDCl}_{3}, 25^{\circ} \mathrm{C}\right): \delta=166.4,164.8,149.8,138.7$, 
138.0, 133.7, 132.8, 130.8, 127.8, 126.2, 62.1, 61.5, 35.2, 14.6, 14.4. MS (70 eV, EI), m/z (\%): 306 (2) [M-H] $]^{+}, 278$ (16), 261 (100), 244 (7), 233 (67), 217 (24), 206 (21), 188 (29), 172 (15), 160 (29), 144 (24), 134 (13), 115 (33), 103 (13), 89 (14), 77 (14), 63 (7), 55 (5). IR (KBr): $\tilde{v}=3429$ (br, w), 2984 (m), 1724 (vs), 1620 (m), 1536 (vs), 1494 (w), 1446 (w), 1408 (w), 1367 (s), 1281 (vs), 1263 (vs), 1152 (s), 1113 (s), 1023 (m), 935 (w), 863 (w), 822 (w), $772(w), 757$ (w), 725 (w). HRMS for $\mathbf{C}_{\mathbf{1 5}} \mathbf{H}_{\mathbf{1 7}} \mathbf{N O}_{\mathbf{6}}$ (307.1056): found: 306.0957 [M-H]

\section{Synthesis of ethyl 4-benzoyl-3-nitrobenzoate (14d)}<smiles>CCOC(=O)c1ccc(C(=O)c2ccccc2)c([N+](=O)[O-])c1</smiles>

Prepared according to TP 3 from ethyl 4-iodo-3-nitrobenzoate (1f) (642 mg, $2.00 \mathrm{mmol}$ ), $\mathrm{PhMgCl}$ (1.2 mL, $2.2 \mathrm{mmol}, 1.9 \mathrm{M}$ in THF), CuCN.2LiCl (2.2 mL, 2.2 mmol, 1.0 M in THF) and benzoyl bromide $(481 \mathrm{mg}, 2.6 \mathrm{mmol})$. Reaction time: $0.5 \mathrm{~h}$. Purification by flash chromatography (pentane/diethyl ether $=4: 1$ ) yielded title compound $\mathbf{1 4 d}(454 \mathrm{mg}, 76 \%)$ as a yellow solid.

mp.: $\quad 93-94{ }^{\circ} \mathrm{C} .{ }^{1} \mathbf{H}-\mathbf{N M R}\left(300 \mathrm{MHz}, \mathrm{CDCl}_{3}, 25^{\circ} \mathrm{C}\right): \delta=8.79\left(\mathrm{~d},{ }^{4} J(\mathrm{H}, \mathrm{H})=1.8 \mathrm{~Hz}, 1 \mathrm{H}\right)$, $8.35\left(\mathrm{dd},{ }^{3} J(\mathrm{H}, \mathrm{H})=8.4 \mathrm{~Hz},{ }^{4} J(\mathrm{H}, \mathrm{H})=1.8 \mathrm{~Hz}, 1 \mathrm{H}\right), 7.68-7.64(\mathrm{~m}, 2 \mathrm{H}), 7.56-7.48(\mathrm{~m}, 2 \mathrm{H})$, 7.41-7.36 (m, $2 \mathrm{H}), 4.42\left(\mathrm{q},{ }^{3} J(\mathrm{H}, \mathrm{H})=7.1 \mathrm{~Hz}, 2 \mathrm{H}\right), 1.38\left(\mathrm{t},{ }^{3} J(\mathrm{H}, \mathrm{H})=7.1 \mathrm{~Hz}, 3 \mathrm{H}\right) .{ }^{13} \mathbf{C}-$ NMR $\left(75 \mathrm{MHz}, \mathrm{CDCl}_{3}, 25^{\circ} \mathrm{C}\right): \delta=193.0,164.2,147.1,140.1,135.9,135.1,134.5,133.5$, 129.6, 129.5, 129.3, 126.0, 62.7, 14.7. MS (70 eV, EI), m/z (\%): 299 (6) [M $\left.\mathrm{M}^{+}\right], 270$ (2), 254 (21), 222 (18), 206 (100), 194 (4), 176 (23), 152 (21), 126 (3), 105 (98), 77 (37), 51 (6). IR (KBr): $\tilde{v}=3422$ (br, w), 2986 (m), 1721 (vs), 1674 (vs), 1597 (w), 1534 (vs), 1492 (m), 1449 (m), 1352 (vs), 1287 (s), 1236 (s), 1163 (s), 1020 (m), 941 (m), 920 (s), 858 (m), 837 (m), 766 (s), 742 (m), 715 (s), 701 (m), 685 (m). HRMS for $\mathbf{C}_{\mathbf{1 6}} \mathbf{H}_{\mathbf{1 3}} \mathbf{N O}_{\mathbf{5}}$ (299.0794): found: 299.0802.

\section{Synthesis of ethyl 3-nitro-4-propionyl-benzoate (14e)}<smiles>CCOC(=O)c1ccc(C(=O)CC)c([N+](=O)[O-])c1</smiles>

Prepared according to TP 3 from ethyl 4-iodo-3-nitrobenzoate (1f) (482 mg, $1.50 \mathrm{mmol}$ ), $\mathrm{PhMgCl}(0.97 \mathrm{~mL}, 1.6 \mathrm{mmol}, 1.7 \mathrm{M}$ in THF), CuCN.2LiCl (1.6 mL, $1.6 \mathrm{mmol}, 1.0 \mathrm{M}$ in THF) and propionyl chloride $(223 \mathrm{mg}, 2.25 \mathrm{mmol})$. Reaction time: $4 \mathrm{~h}$. Purification by flash chromatography (pentane/ethyl acetate $=9: 1)$ furnished title compound $\mathbf{1 4 e}(231 \mathrm{mg}, 61 \%)$ as a yellow oil. 
${ }^{1} \mathbf{H}$-NMR $\left(300 \mathrm{MHz}, \quad \mathrm{CDCl}_{3}, 25^{\circ} \mathrm{C}\right): \delta=8.68\left(\mathrm{~d}, \quad{ }^{4} J(\mathrm{H}, \mathrm{H})=1.8 \mathrm{~Hz}, \quad 1 \mathrm{H}\right), 8.29(\mathrm{dd}$, $\left.{ }^{3} J(\mathrm{H}, \mathrm{H})=8.4 \mathrm{~Hz}, \quad{ }^{4} J(\mathrm{H}, \mathrm{H})=1.8 \mathrm{~Hz}, \quad 1 \mathrm{H}\right), 7.38 \quad\left(\mathrm{~d},{ }^{3} J(\mathrm{H}, \mathrm{H})=8.4 \mathrm{~Hz}, 1 \mathrm{H}\right), 4.38 \quad(\mathrm{q}$, $\left.{ }^{3} J(\mathrm{H}, \mathrm{H})=7.1 \mathrm{~Hz}, 2 \mathrm{H}\right), 2.74\left(\mathrm{q},{ }^{3} J(\mathrm{H}, \mathrm{H})=7.1 \mathrm{~Hz}, 2 \mathrm{H}\right), 1.37\left(\mathrm{t},{ }^{3} J(\mathrm{H}, \mathrm{H})=7.1,3 \mathrm{H}\right), 1.20(\mathrm{t}$, $\left.{ }^{3} J(\mathrm{H}, \mathrm{H})=7.1,3 \mathrm{H}\right) .{ }^{13} \mathbf{C}-\mathrm{NMR}\left(75 \mathrm{MHz}, \mathrm{CDCl}_{3}, 25{ }^{\circ} \mathrm{C}\right): \delta=201.5,162.8,144.7,140.6$,

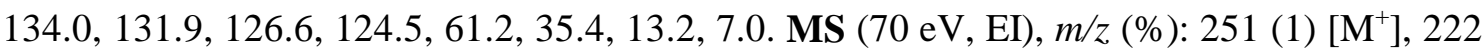
(100), 206 (9), 193 (5), 176 (2), 165 (8), 148 (1), 136 (1), 120 (2), 103 (5). IR (KBr): $\tilde{v}=3094(\mathrm{w}), 2984(\mathrm{~s}), 2942(\mathrm{~m}), 2907(\mathrm{w}), 2880(\mathrm{w}), 1726(\mathrm{vs}), 1622(\mathrm{~m}), 1566(\mathrm{~m}), 1538$ (vs), 1491 (s), 1461 (m), 1403 (m), 1351 (vs), 1311 (vs), 1287 (vs), 1256 (vs), 1211 (vs), 1175 (m), $1155(\mathrm{~s}), 1125(\mathrm{~s}), 1092(\mathrm{~s}), 1069(\mathrm{w}), 1018(\mathrm{~s}), 955(\mathrm{~s}), 920(\mathrm{~m}), 861(\mathrm{~m}), 839(\mathrm{~m}), 803$ (w), 768 (s), 750 (s), 696 (w), 564 (w). HRMS for $\mathbf{C}_{\mathbf{1 2}} \mathbf{H}_{\mathbf{1 3}} \mathbf{N} \mathbf{O}_{\mathbf{5}}$ (251.0794): found: 251.0768.

\section{Synthesis of ethyl 4-(2-methyl-3-oxo-1-cyclopentenyl)-3-nitro-benzoate (14f)}

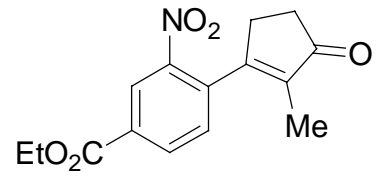

Prepared according to TP 3 from ethyl 4-iodo-3-nitrobenzoate (1f) (482 $\mathrm{mg}, 1.50 \mathrm{mmol}$ ), $\mathrm{PhMgCl}$ (0.97 mL, $1.6 \mathrm{mmol}, 1.7 \mathrm{M}$ in THF), CuCN.2LiCl (1.6 mL, $1.6 \mathrm{mmol}, 1.0 \mathrm{M}$ in THF) and 3-iodo-2-methyl-2-pentenone (500 mg, $2.25 \mathrm{mmol})$. Reaction time: $3 \mathrm{~h}$. Purification by flash chromatography (pentane/diethyl ether $=1: 2)$ yielded compound $\mathbf{1 4 f}(266 \mathrm{mg}, 61 \%)$ as a yellow solid.

mp.: $80-81{ }^{\circ} \mathrm{C} .{ }^{1} \mathbf{H}-\mathrm{NMR}\left(300 \mathrm{MHz}, \mathrm{CDCl}_{3}, 25^{\circ} \mathrm{C}\right): \delta=8.69\left(\mathrm{~d},{ }^{4} \mathrm{~J}(\mathrm{H}, \mathrm{H})=1.8 \mathrm{~Hz}, 1 \mathrm{H}\right)$, $8.29\left(\mathrm{dd},{ }^{3} J(\mathrm{H}, \mathrm{H})=8.2 \mathrm{~Hz},{ }^{4} J(\mathrm{H}, \mathrm{H})=1.8 \mathrm{~Hz}, 1 \mathrm{H}\right), 7.32\left(\mathrm{~d},{ }^{3} J(\mathrm{H}, \mathrm{H})=8.2 \mathrm{~Hz}, 1 \mathrm{H}\right), 4.40(\mathrm{q}$, $\left.{ }^{3} J(\mathrm{H}, \mathrm{H})=7.1 \mathrm{~Hz}, 2 \mathrm{H}\right), 2.79-2.75(\mathrm{~m}, 2 \mathrm{H}), 2.55-2.52(\mathrm{~m}, 2 \mathrm{H}), 1.52(\mathrm{~s}, 3 \mathrm{H}), 1.37(\mathrm{t}$, $\left.{ }^{3} J(\mathrm{H}, \mathrm{H})=7.1 \mathrm{~Hz}, 3 \mathrm{H}\right) .{ }^{13} \mathrm{C}-\mathbf{N M R}\left(300 \mathrm{MHz}, \mathrm{CDCl}_{3}, 25{ }^{\circ} \mathrm{C}\right): \delta=208.4,165.1,164.4,147.6$, 139.3, 137.5, 134.6, 132.5, 130.0, 126.4, 62.5, 34.7, 31.1, 14.6, 9.0. MS (70 eV, EI), m/z (\%): 290 (32) [M+H] $]^{+}, 244$ (74), 234 (57), 218 (100), 204 (73), 192 (83), 176 (50), 144 (24), 141 (36), 128 (37), 115 (60). IR (KBr): $\tilde{v}=3432$ (m), 1708 (vs) 1721 (vs), 1652 (w), 1619 (m), 1533 (s), 1440 (w), 1398 (w), 1388 (w), 1345 (m), 1289 (s), 1252 (s), 1219 (w), 1160 (w), 1112 (m), 1058 (w), 1021 (m), 865 (w), 837 (w), 774 (w), 729 (m), 664 (w). HRMS for $\mathbf{C}_{15} \mathbf{H}_{15} \mathbf{N O}_{5}$ (289.0950): found: $290.1026[\mathrm{M}+\mathrm{H}]^{+}$. $\mathbf{C}_{\mathbf{1 5}} \mathbf{H}_{15} \mathbf{N O}_{5}$ : required: C: 62.28; H: 5.23; $\mathrm{N}: 4.84$; found: C: $61.99 ; \mathrm{H}: 5.37 ; \mathrm{N}: 4.74$.

\section{Synthesis of ethyl 2-(4-cyano-2-nitrobenzyl)acrylate (14g)}<smiles>C=C(Cc1ccc(C#N)cc1[N+](=O)[O-])C(=O)OCC</smiles>

Prepared according to TP 3 from 4-iodo-3-nitrobenzonitrile (1g) (548 mg, $2.00 \mathrm{mmol}$ ), $\mathrm{PhMgCl}(1.1 \mathrm{~mL}, 2.1 \mathrm{mmol}, 2.0 \mathrm{M}$ in THF), CuCN.2LiCl (2.0 mL, $2.0 \mathrm{mmol}, 1.0 \mathrm{M}$ in THF) 
and ethyl (2-bromomethyl) acrylate (463 mg, $2.40 \mathrm{mmol})$. Reaction time: $30 \mathrm{~min}$. Purification by flash chromatography (pentane/CH2CL2 = 4:6) furnished title compound $\mathbf{1 4 g}$ (300 mg, $58 \%$ ) as a yellow solid.

mp.: $\quad 62-63{ }^{\circ} \mathrm{C} .{ }^{1} \mathbf{H}-\mathbf{N M R}\left(300 \mathrm{MHz}, \mathrm{CDCl}_{3}, 25^{\circ} \mathrm{C}\right): \delta=8.23\left(\mathrm{~d},{ }^{4} \mathrm{~J}(\mathrm{H}, \mathrm{H})=1.8 \mathrm{~Hz}, 1 \mathrm{H}\right)$, $7.81\left(\mathrm{dd},{ }^{3} J(\mathrm{H}, \mathrm{H})=8.4 \mathrm{~Hz},{ }^{4} J(\mathrm{H}, \mathrm{H})=1.8 \mathrm{~Hz}, 1 \mathrm{H}\right), 7.56\left(\mathrm{~d},{ }^{3} J(\mathrm{H}, \mathrm{H})=8.4 \mathrm{~Hz}, 1 \mathrm{H}\right), 6.31(\mathrm{~s}$, $1 \mathrm{H}), 5.38(\mathrm{~m}, 1 \mathrm{H}), 4.13\left(\mathrm{q},{ }^{3} J(\mathrm{H}, \mathrm{H})=7.1 \mathrm{~Hz}, 2 \mathrm{H}\right), 3.95(\mathrm{~s}, 2 \mathrm{H}), 1.17\left(\mathrm{t},{ }^{3} J(\mathrm{H}, \mathrm{H})=7.1 \mathrm{~Hz}\right.$, $3 \mathrm{H}) .{ }^{13} \mathbf{C}-\mathbf{N M R}\left(75 \mathrm{MHz}, \mathrm{CDCl}_{3}, 25^{\circ} \mathrm{C}\right): \delta=165.8,149.5,139.1,137.0,135.6,133.4,128.4$, 128.2, 116.5, 112.1, 61.3, 34.9, 14.0. MS (70 eV, EI), $m / z(\%): 261(0.1)\left[\mathrm{M}^{+}\right], 215$ (100), 186 (77), 169 (40), 159 (47),142 (39), 129 (27), 115 (28), 104 (23), 90 (18), 77 (14), 63 (17). IR (KBr): $\tilde{v}=3065$ (m), 1703 (s), 1703 (vs), 1536 (vs), 1361 (s), 1331 (s), 1310 (s), 1211 (m), 1197 (s), 1159 (m), 1027 (m, 965 (m), 928 (m), 849 (m), 826 (m). HRMS for $\mathbf{C}_{\mathbf{1 3}} \mathbf{H}_{\mathbf{1 2}} \mathbf{N}_{\mathbf{2}} \mathbf{O}_{\mathbf{4}}$ (260.0797): found: $261.0887[\mathrm{M}+\mathrm{H}]^{+}$.

\section{Synthesis of ethyl 2-[2-nitro-4-(1-piperidinylcarbonyl)benzyl]acrylate (14h)}

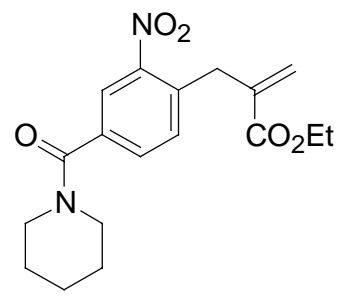

Prepared according to TP 3 from 1-(4-iodo-3-nitrobenzoyl)piperidine (1h) (360 mg, $1.00 \mathrm{mmol}), \mathrm{PhMgCl}(0.63 \mathrm{~mL}, 1.1 \mathrm{mmol}, 1.8 \mathrm{M}$ in THF), CuCN.2LiCl (1.1 mL, $1.1 \mathrm{mmol}$, $1.0 \mathrm{M}$ in THF) and ethyl (2-bromomethyl) acrylate $(386 \mathrm{mg}, 2.00 \mathrm{mmol})$. Reaction time: $2 \mathrm{~h}$. Purification by flash chromatography (pentane/diethyl ether $=1: 2$ ) yielded compound $\mathbf{1 4 h}$ as a pale yellow oil $(270 \mathrm{mg}, 78 \%)$.

${ }^{1} \mathbf{H}$-NMR $\left(300 \mathrm{MHz}, \quad \mathrm{CDCl}_{3}, 25^{\circ} \mathrm{C}\right): \delta=7.94\left(\mathrm{~d},{ }^{4} J(\mathrm{H}, \mathrm{H})=1.8 \mathrm{~Hz}, 1 \mathrm{H}\right), 7.55(\mathrm{dd}$, $\left.{ }^{3} J(\mathrm{H}, \mathrm{H})=8.0 \mathrm{~Hz},{ }^{4} J(\mathrm{H}, \mathrm{H})=1.8 \mathrm{~Hz}, 1 \mathrm{H}\right), 7.40\left(\mathrm{~d},{ }^{3} J(\mathrm{H}, \mathrm{H})=8.0 \mathrm{~Hz}, 1 \mathrm{H}\right), 6.27(\mathrm{~m}, 1 \mathrm{H}), 5.44$ $(\mathrm{m}, 1 \mathrm{H}), 6.05-5.94(\mathrm{~m}, 1 \mathrm{H}), 4.16\left(\mathrm{q},{ }^{3} J(\mathrm{H}, \mathrm{H})=7.1 \mathrm{~Hz}, 2 \mathrm{H}\right), 3.97(\mathrm{~s}, 2 \mathrm{H}), 3.7$ (s_br, $\left.2 \mathrm{H}\right)$, 3.50 (s_br, $2 \mathrm{H}), 1.70-1.45(\mathrm{~m}, 6 \mathrm{H}), 1.23\left(\mathrm{t},{ }^{3} J(\mathrm{H}, \mathrm{H})=7.1 \mathrm{~Hz}, 3 \mathrm{H}\right) .{ }^{13} \mathbf{C}-\mathbf{N M R}(75 \mathrm{MHz}$, $\left.\mathrm{CDCl}_{3}, 25^{\circ} \mathrm{C}\right): \delta=167.5,166.2,149.2,137.9,136.2,134.9,132.6,131.2,127.2,123.5$, 112.5, 61.1, 48.9, 43.4, 34.6, 25.5, 24.4, 14.1. MS (70 eV, EI), m/z (\%): $345(100)\left[\mathrm{M}^{+}\right], 329$ (18), 311 (19), 301 (19), 283 (6), 262 (12), 254 (7), 239 (10), 226 (14), 216 (9), 200 (3), 189 (8), 172 (10), 160 (16), 143 (15), 130 (8), 115 (23), 103 (7), 89 (10), 84 (11), 77 (7), 69 (6), 56 (5). IR (KBr): $\widetilde{v}=2939$ (s), 1715 (vs), 1633 (vs), 1532 (vs), 1498 (s), 1441 (vs), 1351 (vs), 1276 (vs), 1191 (s), 1135 (s), 1124 (m), 1026 (s), 1010 (s), 955 (m), 906 (w), 854 (m), 822 (m), 756 (w), 732 (w), 668 (w). HRMS for $\mathbf{C}_{\mathbf{1 8}} \mathbf{H}_{\mathbf{2 2}} \mathbf{N}_{\mathbf{2}} \mathbf{O}_{\mathbf{5}}$ (346.1529): found: 345.1453 $[\mathrm{M}-\mathrm{H}]^{+}$. 


\section{Synthesis of ethyl 2-(3,5-dimethyl-2-nitrobenzyl)acrylate (14i)}<smiles>C=C(COCC)Cc1cc(C)cc(C)c1[N+](=O)[O-]</smiles>

Prepared according to TP 3 from 2-iodo-4,6-dimethyl-nitrobenzene (1k) (277 mg, $1.00 \mathrm{mmol}), \mathrm{PhMgCl}(0.73 \mathrm{~mL}, 1.1 \mathrm{mmol}, 1.5 \mathrm{M}$ in THF), CuCN.2LiCl (1.1 mL, $1.1 \mathrm{mmol}$, $1.0 \mathrm{M}$ in THF) and ethyl (2-bromomethyl) acrylate $(288 \mathrm{mg}, 1.50 \mathrm{mmol})$. Reaction time: $1 \mathrm{~h}$. Purification by flash chromatography (pentane/diethyl ether $=9: 1$ ) yielded title compound 14i as a yellow oil (200 $\mathrm{mg}, 76 \%)$.

${ }^{1}$ H-NMR $\left(400 \mathrm{MHz}, \quad \mathrm{CDCl}_{3}, \quad 25^{\circ} \mathrm{C}\right): \delta=6.96(\mathrm{~s}, \quad 1 \mathrm{H}), 6.92 \quad(\mathrm{~s}, \quad 1 \mathrm{H}), 6.27 \quad(\mathrm{~d}$, $\left.{ }^{2} J(\mathrm{H}, \mathrm{H})=1.0 \mathrm{~Hz}, 1 \mathrm{H}\right), 5.43\left(\mathrm{~d},{ }^{2} J(\mathrm{H}, \mathrm{H})=1.0 \mathrm{~Hz}, 1 \mathrm{H}\right), 4.16\left(\mathrm{q},{ }^{3} J(\mathrm{H}, \mathrm{H})=7.1 \mathrm{~Hz}, 2 \mathrm{H}\right), 3.60$ $(\mathrm{s}, 2 \mathrm{H}), 2.31(\mathrm{~s}, 3 \mathrm{H}), 2.27(\mathrm{~s}, 3 \mathrm{H}), 1.24\left(\mathrm{t},{ }^{3} J(\mathrm{H}, \mathrm{H})=7.1 \mathrm{~Hz}, 3 \mathrm{H}\right) \cdot{ }^{13} \mathbf{C}-\mathbf{N M R}(100 \mathrm{MHz}$, $\left.\mathrm{CDCl}_{3}, 25^{\circ} \mathrm{C}\right): \delta=166.3,140.4,138.1,130.4,130.3,129.9,129.1,127.1,60.9,36.0,33.5$, 21.1, 17.6, 14.1. MS (70 eV, EI), m/z (\%): 262 (0.2) [M-H] $]^{+}, 217$ (100), 200 (26), 189 (58), 172 (53), 160 (15), 144 (23), 128 (18), 115 (15), 91 (13), 77 (8). IR (KBr): $\tilde{v}=2982$ (w), 1717 (vs), 1603 (m), 1525 (vs), 1443 (m), 1366 (s), 1301 (m), 1255 (m), 1194 (m), 1143 (s), $1026(\mathrm{~m}), 953(\mathrm{w}), 860(\mathrm{~m}), 835$ (m), 817 (w). HRMS for $\mathbf{C}_{\mathbf{1 4}} \mathbf{H}_{\mathbf{1 7}} \mathbf{N O}_{\mathbf{4}}$ (263.1158): found: $262.1094[\mathrm{M}-\mathrm{H}]^{+}$.

\section{Synthesis of 2-allyl-4,6-dimethyl-1-nitrobenzene (14j)}

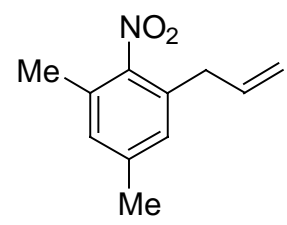

Prepared according to TP 3 from 2-iodo-4,6-dimethyl-nitrobenzene (1k) (277 mg, $1.00 \mathrm{mmol}), \mathrm{PhMgCl}(0.73 \mathrm{~mL}, 1.1 \mathrm{mmol}, 1.5 \mathrm{M}$ in THF), CuCN.2LiCl (1.1 mL, $1.1 \mathrm{mmol}$, $1.0 \mathrm{M}$ in THF) and allyl bromide $(242 \mathrm{mg}, 2.00 \mathrm{mmol})$. Reaction time: $2 \mathrm{~h}$. Purification by flash chromatography (pentane/diethyl ether $=49: 1$ ) yielded compound $\mathbf{1 4} \mathbf{j}$ as a yellow oil (149 mg, 78\%).

${ }^{1}$ H-NMR $\left(400 \mathrm{MHz}, \mathrm{CDCl}_{3}, 25^{\circ} \mathrm{C}\right): \delta=6.95-6.92(\mathrm{~m}, 2 \mathrm{H}), 5.92-5.82(\mathrm{~m}, 1 \mathrm{H}), 5.11-5.05$ $(\mathrm{m}, 2 \mathrm{H}), 3.32\left(\mathrm{~d},{ }^{3} J(\mathrm{H}, \mathrm{H})=6.7 \mathrm{~Hz}, 2 \mathrm{H}\right), 2.32(\mathrm{~s}, 3 \mathrm{H}), 2.27(\mathrm{~s}, 3 \mathrm{H}) .{ }^{13} \mathbf{C}-\mathbf{N M R}(100 \mathrm{MHz}$, $\left.\mathrm{CDCl}_{3}, 25^{\circ} \mathrm{C}\right): \delta=140.5,135.0,131.5,130.0,129.7,128.7,117.1,113.0,35.6,21.1,17.5$.

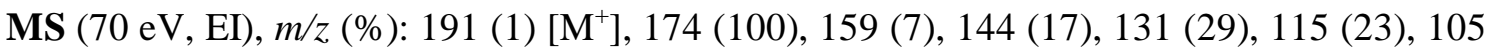
(9), 91 (21), 77 (14), 65 (5), 55 (28). IR (KBr): $\widetilde{v}=2925$ (m), 1603 (w), $1524(\mathrm{vs}), 1441(\mathrm{w})$, 1366 (vs), 1296 (w), 1180 (w), 994 (m), 918 (m), 856 (m), 834 (s), 604 (w). HRMS for $\mathbf{C}_{11} \mathbf{H}_{13} \mathbf{N O}_{2}$ (191.0946): found: 191.0949. 


\section{Synthesis of ethyl 2-(4-iodo-2-nitrobenzyl)acrylate (14k)}

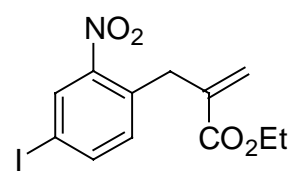

Prepared according to TP 3 from 2,5-diiodo-nitrobenzene (1m) (563 mg, $1.50 \mathrm{mmol}$ ), $\mathrm{PhMgCl}(0.85 \mathrm{~mL}, 1.7 \mathrm{mmol}, 2.0 \mathrm{M}$ in THF), CuCN.2LiCl(1.7 mL, $1.7 \mathrm{mmol}, 1.0 \mathrm{M}$ in THF) and ethyl (2-bromomethyl) acrylate $(345 \mathrm{mg}, 1.80 \mathrm{mmol})$. Reaction time: $45 \mathrm{~min}$. Purification by flash chromatography (pentane/ $\mathrm{CH}_{2} \mathrm{Cl}_{2}=2: 1$ ) yielded compound $\mathbf{1 4 k}(401 \mathrm{mg}, 74 \%)$ as a yellow oil.

${ }^{1} \mathbf{H}$-NMR $\left(300 \mathrm{MHz}, \mathrm{CDCl}_{3}, 25{ }^{\circ} \mathrm{C}\right): \delta=8.16\left(\mathrm{~d}, \quad{ }^{4} J(\mathrm{H}, \mathrm{H})=1.8 \mathrm{~Hz}, 1 \mathrm{H}\right), 7.77(\mathrm{dd}$, $\left.{ }^{3} J(\mathrm{H}, \mathrm{H})=8.4 \mathrm{~Hz},{ }^{4} J(\mathrm{H}, \mathrm{H})=1.8 \mathrm{~Hz}, 1 \mathrm{H}\right), 7.04 \quad\left(\mathrm{~d},{ }^{3} J(\mathrm{H}, \mathrm{H})=8.4 \mathrm{~Hz}, 1 \mathrm{H}\right), 6.21 \quad(\mathrm{~d}$, $\left.{ }^{2} J(\mathrm{H}, \mathrm{H})=0.9 \mathrm{~Hz}, 1 \mathrm{H}\right), 5.38\left(\mathrm{~d},{ }^{2} J(\mathrm{H}, \mathrm{H})=0.9 \mathrm{~Hz}, 1 \mathrm{H}\right), 4.10\left(\mathrm{q},{ }^{3} J(\mathrm{H}, \mathrm{H})=7.1 \mathrm{~Hz}, 2 \mathrm{H}\right), 3.84$ $(\mathrm{s}, 2 \mathrm{H}), 1.18\left(\mathrm{t},{ }^{3} J(\mathrm{H}, \mathrm{H})=7.1 \mathrm{~Hz}, 3 \mathrm{H}\right) .{ }^{13} \mathrm{C}-\mathbf{N M R}\left(75 \mathrm{MHz}, \mathrm{CDCl}_{3}, 25{ }^{\circ} \mathrm{C}\right): \delta=165.1$, $148.8,140.7,136.7,132.8,132.3,132.3,126.2,89.8,60.1,33.4,13.1 . ~ M S(70 \mathrm{eV}, \mathrm{EI}), \mathrm{m} / z$ (\%): 360 (7) $\left[\mathrm{M}^{+}\right], 344$ (10), 332 (100), 316 (100), 304 (12), 298 (28), 287 (78), 270 (78), 261 (58), 230 (19), 205 (32), 177 (11), 161 (72), 145 (21), 133 (66), 117 (22), 102 (29), 89 (58), 77 (34), 63 (41). IR (KBr): $\tilde{v}=2981$ (w), 1714 (vs), 1529 (vs), 1475 (m), 1349 (vs), 1299 (m), 1137 (s), 1025 (m), 953 (m), 810 (m), 727 (w), 701 (w). HRMS for $\mathbf{C}_{\mathbf{1 2}} \mathbf{H}_{\mathbf{1 2}} \mathbf{I N O}_{\mathbf{4}}$ (360.9811): found: $359.9765[\mathrm{M}-\mathrm{H}]^{+}$. $\mathbf{C}_{\mathbf{1 2}} \mathbf{H}_{\mathbf{1 2}} \mathbf{I N O}_{\mathbf{4}}$ : required: C: 39.91; H: 3.35; $\mathrm{N}: 3,88$; found: C: $39.90 ; \mathrm{H}: 3.10 ; \mathrm{N}: 3.86$.

\section{Synthesis of ethyl 2-(4-benzoyl-2-nitrobenzyl)acrylate (14l)}<smiles>C=C(COCC)Cc1ccc(C(=O)c2ccccc2)cc1[N+](=O)[O-]</smiles>

Prepared according to TP 3 from 4-iodo-3-nitrobenzophenone (1o) (353 mg, $1.00 \mathrm{mmol}$ ), $\mathrm{PhMgCl}(0.73 \mathrm{~mL}, 1.1 \mathrm{mmol}, 1.5 \mathrm{M}$ in THF), CuCN.2LiCl (1.1 mL, $1.1 \mathrm{mmol}, 1.0 \mathrm{M}$ in THF) and ethyl (2-bromomethyl) acrylate $(288 \mathrm{mg}, 1.50 \mathrm{mmol})$. Reaction time: $1 \mathrm{~h}$. Purification by flash chromatography (pentane/diethyl ether $=9: 1$ ) yielded title compound $\mathbf{1 4 l}$ as a yellow oil (329 mg, 97\%).

${ }^{1} \mathbf{H}$-NMR $\left(300 \mathrm{MHz}, \mathrm{CDCl}_{3}, 25^{\circ} \mathrm{C}\right): \delta=8.34\left(\mathrm{~d},{ }^{4} J(\mathrm{H}, \mathrm{H})=1.8 \mathrm{~Hz}, 1 \mathrm{H}\right), 7.99(\mathrm{dd}$, $\left.{ }^{3} J(\mathrm{H}, \mathrm{H})=8.0 \mathrm{~Hz},{ }^{4} J(\mathrm{H}, \mathrm{H})=1.8 \mathrm{~Hz}, 1 \mathrm{H}\right), 7.84-7.78(\mathrm{~m}, 2 \mathrm{H}), 7.69-7.63(\mathrm{~m}, 1 \mathrm{H}), 7.57-7.51$ $(\mathrm{m}, 3 \mathrm{H}), 6.85\left(\mathrm{~d},{ }^{2} J(\mathrm{H}, \mathrm{H})=0.9 \mathrm{~Hz}, 1 \mathrm{H}\right), 5.54\left(\mathrm{~d},{ }^{2} J(\mathrm{H}, \mathrm{H})=0.9 \mathrm{~Hz}, 1 \mathrm{H}\right), 4.21 \quad(\mathrm{q}$, $\left.{ }^{3} J(\mathrm{H}, \mathrm{H})=7.1 \mathrm{~Hz}, 2 \mathrm{H}\right), 4.07(\mathrm{~s}, 2 \mathrm{H}), 1.28\left(\mathrm{t},{ }^{3} J(\mathrm{H}, \mathrm{H})=7.1 \mathrm{~Hz}, 3 \mathrm{H}\right) .{ }^{13} \mathbf{C}-\mathbf{N M R}(75 \mathrm{MHz}$, $\left.\mathrm{CDCl}_{3}, 25^{\circ} \mathrm{C}\right): \delta=194.2,166.4,149.7,138.2,138.0,137.5,136.8,134.0,133.6,132.9$, $130.3,129.1,127.9,126.6,61.5,35.2$, 14.6. MS (70 eV, EI), $m / z(\%): 338$ (1) $[\mathrm{M}-\mathrm{H}]^{+}, 310$ 
(8), 293 (27), 265 (14), 248 (8), 189 (5), 160 (4), 144 (5), 115 (8), 105 (100), 77 (55). IR (KBr): $\widetilde{v}=1715$ (vs), 1665 (vs), 1616 (s), 1534 (vs), 147 (s), 1353 (s), 1318 (s), 1301 (s), 1278 (vs), 1191 (s), 1180 (s), 1151 (s), 721 (m), 700 (m). HRMS for $\mathbf{C}_{\mathbf{1 4}} \mathbf{H}_{\mathbf{1 7}} \mathbf{N O}_{\mathbf{4}}$ (339.1107): found: $338.1025[\mathrm{M}-\mathrm{H}]^{+} . \mathbf{C}_{\mathbf{1 9}} \mathbf{H}_{\mathbf{1 7}} \mathbf{N O}_{\mathbf{5}}$ : required: $\mathrm{C}: 67.25 ; \mathrm{H}: 5.05 ; \mathrm{N}: 4.13$; found: $\mathrm{C}: 67.25$; H: 5.30; N: 4.00 .

\section{Synthesis of compounds 22}

\section{Synthesis of ethyl 2-nitro[1,1'-biphenyl]-4-carboxylate (22a)}

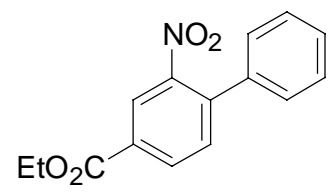

Prepared according to TP 4 from ethyl 4-iodo-3-nitrobenzoate (1f) (482 mg, $1.50 \mathrm{mmol}$ ), $\mathrm{PhMgCl}\left(0.89 \mathrm{~mL}, 1.7 \mathrm{mmol}, 1.9 \mathrm{M}\right.$ in THF), $\mathrm{ZnBr}_{2}(1.7 \mathrm{~mL}, 1.7 \mathrm{mmol}, 1.0 \mathrm{M}$ in THF), $\mathrm{Pd}(\mathrm{dba})_{2}(44.0 \mathrm{mg}, 0.075 \mathrm{mmol})$ and $\mathrm{tfp}(35 \mathrm{mg}, 0.15 \mathrm{mmol})$. Reaction time: $3 \mathrm{~h}$. Purification by flash chromatography (pentane/diethyl ether $=9: 1$ ) yielded biphenyl 22a as a yellow oil (317 mg, 78\%).

${ }^{1} \mathbf{H}$-NMR $\left(300 \mathrm{MHz}, \mathrm{CDCl}_{3}, \quad 25{ }^{\circ} \mathrm{C}\right): \delta=8.47 \quad\left(\mathrm{~d},{ }^{4} J(\mathrm{H}, \mathrm{H})=1.8 \mathrm{~Hz}, 1 \mathrm{H}\right), 8.25(\mathrm{dd}$, $\left.{ }^{3} J(\mathrm{H}, \mathrm{H})=8.0 \mathrm{~Hz},{ }^{4} J(\mathrm{H}, \mathrm{H})=1.8 \mathrm{~Hz}, 1 \mathrm{H}\right), 7.53\left(\mathrm{~d},{ }^{3} J(\mathrm{H}, \mathrm{H})=8.0 \mathrm{~Hz}, 1 \mathrm{H}\right), 7.46-7.40(\mathrm{~m}, 3 \mathrm{H})$, 7.34-7.31 (m, $2 \mathrm{H}), 4.44\left(\mathrm{q},{ }^{3} J(\mathrm{H}, \mathrm{H})=7.1 \mathrm{~Hz}, 2 \mathrm{H}\right), 1.43\left(\mathrm{t},{ }^{3} J(\mathrm{H}, \mathrm{H})=7.1 \mathrm{~Hz}, 3 \mathrm{H}\right) .{ }^{13} \mathrm{C}-$ NMR $\left(75 \mathrm{MHz}, \mathrm{CDCl}_{3}, 25{ }^{\circ} \mathrm{C}\right): \delta=164.3,149.2,140.2,136.8,132.8,132.1,130.7,128.8$, 127.7, 125.1, 113.2, 61.8, 14.3. MS (70 eV, EI), $m / z(\%): 271(23)\left[\mathrm{M}^{+}\right], 254$ (49), 243 (100), 226 (61), 214 (22), 198 (38), 187 (45), 182 (12), 170 (51), 151 (91), 142 (30), 126 (9), 115 (26), 75 (5). IR (KBr): $\tilde{v}=2984$ (m), 1723 (vs), 1618 (m), 1535 (vs), 1447 (m), 1360 (s), 1303 (s), 1285 (vs), 1236 (s), 1153 (m), 1113 (s), 1008 (m), 918 (w), 860 (m), 836 (w), 770 (m), 752 (s), 699 (s), 677 (w). HRMS for $\mathbf{C}_{\mathbf{1 5}} \mathbf{H}_{\mathbf{1 3}} \mathbf{N O}_{\mathbf{4}}$ (271.0845): found: 271.0846.

\section{Synthesis of 4'-nitro-2,4-dinitro-biphenyl (22b)}

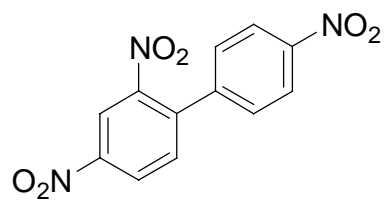

Prepared according to TP 4 from 1-iodo-2,4-dinitrobenzene (1b) (441 mg, $1.50 \mathrm{mmol}$ ), mesitylmagnesium bromide ( $2.5 \mathrm{~mL}, 1.7 \mathrm{mmol}, 0.72 \mathrm{M}$ in THF), $\mathrm{ZnBr}_{2}(1.7 \mathrm{~mL}, 1.7 \mathrm{mmol}$, $1.0 \mathrm{M}$ in THF), $\mathrm{Pd}(\mathrm{dba})_{2}(39.5 \mathrm{mg}, 0.075 \mathrm{mmol})$, tfp $(35 \mathrm{mg}, 0.15 \mathrm{mmol})$ and ethyl 4-iodonitrobenzene $(560 \mathrm{mg}, 2.25 \mathrm{mmol})$. Reaction time: $4 \mathrm{~h}$. Purification by flash chromatography (pentane/ethyl acetate $=5: 1$ ) yielded biphenyl 22b as a yellow solid (240 $\mathrm{mg}, 55 \%)$. 
mp.: $\quad 177-178^{\circ} \mathrm{C} .{ }^{1} \mathbf{H}-\mathbf{N M R}\left(300 \mathrm{MHz}, \mathrm{CDCl}_{3}, 25^{\circ} \mathrm{C}\right): \delta=8.79\left(\mathrm{~d},{ }^{4} \mathrm{~J}(\mathrm{H}, \mathrm{H})=2.2 \mathrm{~Hz}, 1 \mathrm{H}\right)$, $8.49\left(\mathrm{dd},{ }^{3} J(\mathrm{H}, \mathrm{H})=8.4 \mathrm{~Hz},{ }^{4} J(\mathrm{H}, \mathrm{H})=2.4 \mathrm{~Hz}, 1 \mathrm{H}\right), 8.28\left(\mathrm{~d},{ }^{3} J(\mathrm{H}, \mathrm{H})=8.8 \mathrm{~Hz}, 2 \mathrm{H}\right), 7.63(\mathrm{~d}$, $\left.{ }^{3} J(\mathrm{H}, \mathrm{H})=8.4 \mathrm{~Hz}, 1 \mathrm{H}\right), 7.46\left(\mathrm{~d},{ }^{3} \mathrm{~J}(\mathrm{H}, \mathrm{H})=8.8 \mathrm{~Hz}, 2 \mathrm{H}\right) .{ }^{13} \mathbf{C}-\mathbf{N M R}\left(75 \mathrm{MHz}, \mathrm{CDCl}_{3}, 25{ }^{\circ} \mathrm{C}\right)$ : $\delta=147.6,147.4,146.8,140.9,139.2,132.1,127.9,126.1,123.2,119.2 . \mathbf{M S}(70 \mathrm{eV}, \mathrm{EI}), \mathrm{m} / \mathrm{z}$ (\%): 298 (12) $\left[\mathrm{M}^{+}\right], 272$ (36), 242 (47), 215 (70), 169 (92), 150 (100), 139 (68). IR (KBr): $\tilde{v}=3436(\mathrm{~m}), 3099(\mathrm{~m}), 3052$ (w), 1598 (s), 1532 (vs), 1476 (w), 1349 (vs), 1291 (w), $1110(\mathrm{w}), 1078$ (w), 1006 (w), 917 (w), 858 (s), 836 (m), 760 (m), 745 (m), 723 (w), 700 (m), 537 (w). HRMS for $\mathbf{C}_{\mathbf{1 2}} \mathbf{H}_{7} \mathbf{N}_{\mathbf{3}} \mathbf{O}_{\mathbf{6}}$ (289.0335): found: 289.0339 .

\section{Synthesis of ethyl 2',4'-dinitro-biphenyl-4-carboxylate (22c)}

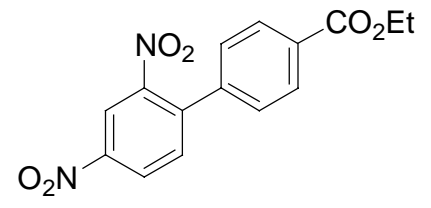

Prepared according to TP 4 from 1-iodo-2,4-dinitrobenzene (1b) (441 mg, $1.50 \mathrm{mmol}$ ), mesitylmagnesium bromide $\left(2.5 \mathrm{~mL}, 1.7 \mathrm{mmol}, 0.72 \mathrm{M}\right.$ in THF), $\mathrm{ZnBr}_{2}(1.7 \mathrm{~mL}, 1.7 \mathrm{mmol}$, $1.0 \mathrm{M}$ in THF), Pd(dba) 2 (39.5 mg, $0.075 \mathrm{mmol})$, tfp $(35 \mathrm{mg}, 0.15 \mathrm{mmol})$ and ethyl 4iodobenzoate (455 mg, $1.65 \mathrm{mmol}$ ). Reaction time: $3 \mathrm{~h}$. Purification by flash chromatography (pentane/diethyl ether $=4: 1)$ yielded biphenyl 22c as a yellow solid $(320 \mathrm{mg}, 68 \%)$.

mp.: $\quad 121-122^{\circ} \mathrm{C} .{ }^{1} \mathbf{H}-\mathrm{NMR}\left(300 \mathrm{MHz}, \mathrm{CDCl}_{3}, 25^{\circ} \mathrm{C}\right): \delta=8.70\left(\mathrm{~d},{ }^{4} \mathrm{~J}(\mathrm{H}, \mathrm{H})=2.4 \mathrm{~Hz}, 1 \mathrm{H}\right)$, $8.43\left(\mathrm{dd},{ }^{3} J(\mathrm{H}, \mathrm{H})=8.4 \mathrm{~Hz},{ }^{4} J(\mathrm{H}, \mathrm{H})=2.4 \mathrm{~Hz}, 1 \mathrm{H}\right), 8.08\left(\mathrm{~d},{ }^{3} J(\mathrm{H}, \mathrm{H})=8.2 \mathrm{~Hz}, 2 \mathrm{H}\right), 7.61(\mathrm{~d}$, $\left.{ }^{3} J(\mathrm{H}, \mathrm{H})=8.4 \mathrm{~Hz}, 1 \mathrm{H}\right), 7.34\left(\mathrm{~d},{ }^{3} J(\mathrm{H}, \mathrm{H})=8.2 \mathrm{~Hz}, 2 \mathrm{H}\right), 4.41\left(\mathrm{q},{ }^{3} J(\mathrm{H}, \mathrm{H})=7.1 \mathrm{~Hz}, 2 \mathrm{H}\right), 1.39$ $\left(\mathrm{t},{ }^{3} J(\mathrm{H}, \mathrm{H})=7.1 \mathrm{~Hz}, 3 \mathrm{H}\right) .{ }^{13} \mathrm{C}-\mathrm{NMR}\left(75 \mathrm{MHz}, \mathrm{CDCl}_{3}, 25{ }^{\circ} \mathrm{C}\right): \delta=164.7,147.9,146.3$, 140.4, 138.6, 132.1, 130.5, 129.2, 126.8, 125.7, 118.9, 60.4, 13.3. MS (70 eV, EI), m/z (\%): 316 (2) [M+ $\mathrm{M}^{+}, 288$ (50), 271 (100), 260 (22), 242 (12), 225 (32), 215 (20), 179 (53), 169 (16), 151 (33), 139 (20). IR (KBr): $\tilde{v}=3106$ (w), 2988 (w), 1717 (vs), 1602 (m), 1530 (vs), 1416 (w), 1352 (s), 1298 (m), 1286 (s), 1189 (w), 1134 (w), 1106 (m), 1077 (w), 1025 (w), 1007 (w), 910 (w), 846 (w), 836 (w), 776 (w), 763 (w), 741 (m), 705 (w). HRMS for $\mathbf{C}_{\mathbf{1 5}} \mathbf{H}_{\mathbf{1 2}} \mathbf{N}_{\mathbf{2}} \mathbf{O}_{\mathbf{6}}$ (316.0695): found: 316.0685. $\mathbf{C}_{\mathbf{1 5}} \mathbf{H}_{\mathbf{1 2}} \mathbf{N}_{\mathbf{2}} \mathbf{O}_{\mathbf{6}}$ : required: C: 56.96; H: 3.82; N: 8.86; found: C: 56.66; H: 3.92; N: 8.69.

\section{Synthesis of 4'-chlor-2,4-dinitro-biphenyl (22d)}

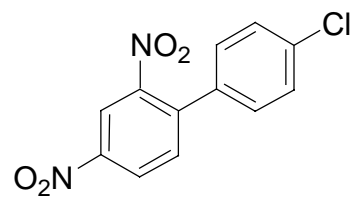

Prepared according to TP 4 from 1-iodo-2,4-dinitrobenzene (1b) (441 mg, $1.50 \mathrm{mmol}$ ), mesitylmagnesium bromide $\left(2.5 \mathrm{~mL}, 1.7 \mathrm{mmol}, 0.72 \mathrm{M}\right.$ in THF), $\mathrm{ZnBr}_{2}$ (1.7 mL, $1.7 \mathrm{mmol}$, $1.0 \mathrm{M}$ in THF), Pd(dba $)_{2}(39.5 \mathrm{mg}, 0.075 \mathrm{mmol}), \mathrm{tfp}(35 \mathrm{mg}, 0.15 \mathrm{mmol})$ and 4-chloro- 
iodobenzene (536 mg, $2.25 \mathrm{mmol})$. Reaction time: $5 \mathrm{~h}$. Purification by flash chromatography (pentane/diethyl ether $=9: 1)$ yielded biphenyl 22d as a pale yellow solid $(228 \mathrm{mg}, 55 \%)$.

mp.: $\quad 80-81{ }^{\circ} \mathrm{C} .{ }^{1} \mathbf{H}-\mathbf{N M R}\left(300 \mathrm{MHz}, \mathrm{CDCl}_{3}, 25^{\circ} \mathrm{C}\right): \delta=8.65\left(\mathrm{~d},{ }^{4} J(\mathrm{H}, \mathrm{H})=2.2 \mathrm{~Hz}, 1 \mathrm{H}\right)$, $8.40\left(\mathrm{dd},{ }^{3} J(\mathrm{H}, \mathrm{H})=8.3 \mathrm{~Hz},{ }^{4} J(\mathrm{H}, \mathrm{H})=2.2 \mathrm{~Hz}, 1 \mathrm{H}\right), 7.58\left(\mathrm{~d},{ }^{3} J(\mathrm{H}, \mathrm{H})=8.3 \mathrm{~Hz}, 1 \mathrm{H}\right), 7.59-7.33$ $(\mathrm{m}, 2 \mathrm{H}), 7.22-7-18(\mathrm{~m}, 2 \mathrm{H}) .{ }^{13} \mathbf{C}-\mathbf{N M R}\left(75 \mathrm{MHz}, \mathrm{CDCl}_{3}, 25{ }^{\circ} \mathrm{C}\right): \delta=147.9,146.1,140.1$, 135.0, 132.1, 128.6, 128.0, 127.4, 125.6, 118.9. MS (70 eV, EI), $m / z(\%): 278$ (74) $\left[\mathrm{M}^{+}\right], 250$ (29), 243 (57), 233 (27), 215 (41), 186 (37), 169 (61), 151 (100), 139 (60). IR (KBr): $\tilde{v}=3103(\mathrm{w}), 1651(\mathrm{w}), 1603$ (s), 1541 (vs), 1528 (vs), 1472 (m), 1351 (vs), 1192 (w), 1150 (w), 1092 (m), $1074(\mathrm{~m}), 984(\mathrm{w}), 1006$ (m), 909 (m), 853 (w), 834 (s), 768 (w), 747 (m), 728 (m), 716 (m), 693 (w), 549 (w), 416 (w). HRMS for $\mathbf{C}_{\mathbf{1 2}} \mathbf{H}_{7} \mathbf{C I N}_{\mathbf{2}} \mathbf{O}_{\mathbf{4}}$ (278.0094): found: 278.0068 .

\section{Synthesis of ethyl 2,4'-dinitro[1,1'-biphenyl]-4-carboxylate (22e)}<smiles>CCOC(=O)c1ccc(-c2ccc([N+](=O)[O-])cc2)c([N+](=O)[O-])c1</smiles>

Prepared according to TP 4 from ethyl 4-iodo-3-nitrobenzoate (1f) (321 mg, $1.00 \mathrm{mmol}$ ), mesitylmagnesium bromide $\left(1.3 \mathrm{~mL}, 1.1 \mathrm{mmol}, 0.85 \mathrm{M}\right.$ in THF), $\mathrm{ZnBr}_{2}(1.1 \mathrm{~mL}, 1.1 \mathrm{mmol}$, $1.0 \mathrm{M}$ in THF), $\mathrm{Pd}(\mathrm{dba})_{2}(29 \mathrm{mg}, 0.05 \mathrm{mmol})$, tfp $(23 \mathrm{mg}, 0.10 \mathrm{mmol})$ and 4-iodo-1nitrobenzene $(374 \mathrm{mg}, 1.50 \mathrm{mmol})$. Reaction time: $5 \mathrm{~h}$ at $\mathrm{rt}$. Purification by flash chromatography (pentane/diethyl ether $=3: 1$ ) yielded biphenyl $22 \mathrm{e}$ as a pale yellow solid (269 mg, 85\%).

mp.: $\quad 124.5-125{ }^{\circ} \mathrm{C} .{ }^{1} \mathbf{H}-\mathbf{N M R}\left(300 \mathrm{MHz}, \mathrm{CDCl}_{3}, 25^{\circ} \mathrm{C}\right): \delta=8.61\left(\mathrm{~d},{ }^{4} J(\mathrm{H}, \mathrm{H})=2.0 \mathrm{~Hz}\right.$, $1 \mathrm{H}), 8.34-8.28(\mathrm{~m}, 3 \mathrm{H}), 7.54-7.47(\mathrm{~m}, 3 \mathrm{H}), 4.44\left(\mathrm{q},{ }^{3} J(\mathrm{H}, \mathrm{H})=7.1 \mathrm{~Hz}, 2 \mathrm{H}\right), 1.43(\mathrm{t}$, $\left.{ }^{3} J(\mathrm{H}, \mathrm{H})=7.1 \mathrm{~Hz}, 3 \mathrm{H}\right) .{ }^{13} \mathrm{C}-\mathrm{NMR}\left(75 \mathrm{MHz}, \mathrm{CDCl}_{3}, 25^{\circ} \mathrm{C}\right): \delta=163.9,148.6,147.9,143.3$, 138.1, 133.4, 132.1, 131.9, 128.9, 125.6, 123.9, 62.1, 14.2. MS (70 eV, EI), $m / z(\%): 316(51)$ $\left[\mathrm{M}^{+}\right], 299$ (50), 271 (88), 255 (9), 242 (53), 225 (46), 214 (72), 195 (36), 179 (72), 168 (26), 150 (100), 139 (67), 125 (13), 115 (21), 99 (9), 75 (24), 63 (10). IR (KBr): $\tilde{v}=1722$ (vs), 1530 (s), 1517 (s), 1353 (vs), 1307 (s), 1286 (s), 1242 (m), 1126 (m), 1110 (m), 857 (m), 850 (m), 749 (m). HRMS for $\mathbf{C}_{\mathbf{1 5}} \mathbf{H}_{\mathbf{1 2}} \mathbf{N}_{\mathbf{2}} \mathbf{O}_{\mathbf{6}}$ (316.0695): found: 316.0686. $\mathbf{C}_{\mathbf{1 5}} \mathbf{H}_{\mathbf{1 2}} \mathbf{N}_{\mathbf{2}} \mathbf{O}_{\mathbf{6}}$ : required: C: $56.96 ; \mathrm{H}: 3.82 ; \mathrm{N}: 8.86$; found: C: $56.69 ; \mathrm{H}: 3.74 ; \mathrm{N}: 8.78$.

\section{Synthesis of ethyl 3'-methoxy-2-nitro[1,1'-biphenyl]-4-carboxylate (22f)}<smiles>CCOC(=O)c1ccc(-c2cccc(OC)c2)c([N+](=O)[O-])c1</smiles> 
Prepared according to TP 4 from ethyl 4-iodo-3-nitrobenzoate (1f) (321 mg, $1.00 \mathrm{mmol}$ ), mesitylmagnesium bromide $\left(1.3 \mathrm{~mL}, 1.1 \mathrm{mmol}, 0.85 \mathrm{M}\right.$ in THF), $\mathrm{ZnBr}_{2}$ (1.1 mL, $1.1 \mathrm{mmol}$, $1.0 \mathrm{M}$ in THF), Pd(dba) 2 (29 mg, $0.05 \mathrm{mmol}), \mathrm{tfp}(23 \mathrm{mg}, 0.10 \mathrm{mmol})$ and 3-iodoanisol (468 mg, $2.00 \mathrm{mmol})$. Reaction time: $4 \mathrm{~h}$. Purification by flash chromatography (pentane/diethyl ether = 9:1) yielded biphenyl $22 f$ as a colorless liquid $(240 \mathrm{mg}, 80 \%)$.

${ }^{1} \mathbf{H}-\mathbf{N M R}\left(300 \mathrm{MHz}, \mathrm{CDCl}_{3}, 25{ }^{\circ} \mathrm{C}\right): \delta=8.49\left(\mathrm{~d},{ }^{4} J(\mathrm{H}, \mathrm{H})=1.8 \mathrm{~Hz}, 1 \mathrm{H}\right), 8.27 \quad(\mathrm{dd}$,

$\left.{ }^{3} J(\mathrm{H}, \mathrm{H})=8.4 \mathrm{~Hz},{ }^{4} J(\mathrm{H}, \mathrm{H})=1.8 \mathrm{~Hz}, 1 \mathrm{H}\right), 7.56\left(\mathrm{~d},{ }^{3} J(\mathrm{H}, \mathrm{H})=8.4 \mathrm{~Hz}, 1 \mathrm{H}\right), 7.37 \quad(\mathrm{t}$, $\left.{ }^{3} J(\mathrm{H}, \mathrm{H})=8.4 \mathrm{~Hz}, 1 \mathrm{H}\right), 6.98\left(\mathrm{dd},{ }^{3} J(\mathrm{H}, \mathrm{H})=8.0 \mathrm{~Hz},{ }^{4} J(\mathrm{H}, \mathrm{H})=1.8 \mathrm{~Hz}, 1 \mathrm{H}\right), 6.93-6.87(\mathrm{~m}$, $2 \mathrm{H}), 4.46\left(\mathrm{q},{ }^{3} J(\mathrm{H}, \mathrm{H})=7.1 \mathrm{~Hz}, 2 \mathrm{H}\right), 3.84(\mathrm{~s}, 3 \mathrm{H}), 1.45\left(\mathrm{t},{ }^{3} J(\mathrm{H}, \mathrm{H})=7.1 \mathrm{~Hz}, 3 \mathrm{H}\right) .{ }^{13} \mathbf{C}-\mathbf{N M R}$ $\left(75 \mathrm{MHz}, \mathrm{CDCl}_{3}, 25{ }^{\circ} \mathrm{C}\right): \delta=164.3,159.8,149.3,140.0,132.7,132.0,130.8,129.9,128.9$, 125.0, 120.1, 114.3, 113.5, 61.8, 55.3, 14.3. MS (70 eV, EI), $m / z(\%): 301(100)\left[\mathrm{M}^{+}\right], 284$ (8), 273 (55), 256 (51), 242 (23), 230 (27), 214 (23), 202 (21), 196 (14), 183 (20), 170 (28), 152 (26), 139 (66), 127 (17), 115 (10), 101 (4), 89 (7), 76 (8), 63 (7), 55 (12). IR (KBr): $\tilde{v}=1728$ (vs), 1663 (m), 1538 (vs), 1355 (s), 1318 (m), 1279 (vs), 1239 (vs), 1133 (m), 828 (m), 716 (m). HRMS for $\mathbf{C}_{\mathbf{1 6}} \mathbf{H}_{\mathbf{1 5}} \mathbf{N O}_{\mathbf{5}}$ (301.0950): found: 301.0954.

\section{Synthesis of ethyl 4'-methoxy-2-nitro[1,1'-biphenyl]-4-carboxylate (22g)}

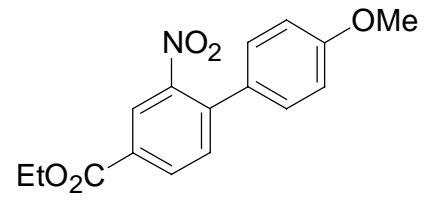

Prepared according to TP 4 from ethyl 4-iodo-3-nitrobenzoate (1f) (482 $\mathrm{mg}, 1.50 \mathrm{mmol}$ ), mesitylmagnesium bromide $\left(2.3 \mathrm{~mL}, 1.6 \mathrm{mmol}, 0.70 \mathrm{M}\right.$ in THF), $\mathrm{ZnBr}_{2}$ (1.6 mL, $1.6 \mathrm{mmol}$, $1.0 \mathrm{M}$ in THF), $\mathrm{Pd}(\mathrm{dba})_{2}(44.0 \mathrm{mg}, 0.075 \mathrm{mmol}), \mathrm{tfp}(35 \mathrm{mg}, 0.15 \mathrm{mmol})$ and 4-iodoanisole $(523 \mathrm{mg}, \quad 2.25 \mathrm{mmol})$. Reaction time: $3 \mathrm{~h}$. Purification by flash chromatography (pentane/ethyl acetate $=9: 1)$ yielded biphenyl 22g as a yellow oil $(220 \mathrm{mg}, 49 \%)$.

${ }^{1}$ H-NMR $\left(300 \mathrm{MHz}, \mathrm{CDCl}_{3}, 25^{\circ} \mathrm{C}\right): \delta=8.32\left(\mathrm{~d},{ }^{4} J(\mathrm{H}, \mathrm{H})=1.8 \mathrm{~Hz}, 1 \mathrm{H}\right), 8.22(\mathrm{dd}$, $\left.{ }^{3} J(\mathrm{H}, \mathrm{H})=8.0 \mathrm{~Hz},{ }^{4} J(\mathrm{H}, \mathrm{H})=1.8 \mathrm{~Hz}, 1 \mathrm{H}\right), 7.51 \quad\left(\mathrm{~d},{ }^{3} J(\mathrm{H}, \mathrm{H})=8.0 \mathrm{~Hz}, 1 \mathrm{H}\right), 7.26 \quad(\mathrm{~d}$, $\left.{ }^{3} J(\mathrm{H}, \mathrm{H})=8.8 \mathrm{~Hz}, 2 \mathrm{H}\right), 6.95\left(\mathrm{~d},{ }^{3} J(\mathrm{H}, \mathrm{H})=8.8 \mathrm{~Hz}, 2 \mathrm{H}\right), 4.41\left(\mathrm{q},{ }^{3} J(\mathrm{H}, \mathrm{H})=7.1 \mathrm{~Hz}, 2 \mathrm{H}\right), 3.83$ $(\mathrm{s}, 3 \mathrm{H}), 1.42\left(\mathrm{t},{ }^{3} \mathrm{~J}(\mathrm{H}, \mathrm{H})=7.1 \mathrm{~Hz}, 3 \mathrm{H}\right) .{ }^{13} \mathrm{C}-\mathbf{N M R}\left(75 \mathrm{MHz}, \mathrm{CDCl}_{3}, 25{ }^{\circ} \mathrm{C}\right): \delta=164.4$, $160.2,149.2,139.7,132.6,131.9,130.2,129.1,128.4,125.1,114.4,61.8,55.3,14.3 . \mathrm{MS}$

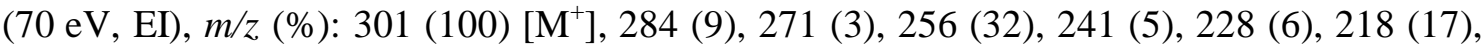
190 (9), 182 (14), 167 (12), 156 (5), 139 (57), 127 (4), 113 (4), 89 (4). IR (KBr): $\widetilde{v}=1722$ (vs), 1675 (s), 1534 (s), 1352 (s), 1315 (m), 1287 (vs), 1237 (vs), 1164 (m), 764 (w), 716 (m), 687 (m). HRMS for $\mathbf{C}_{\mathbf{1 6}} \mathbf{H}_{\mathbf{1 5}} \mathbf{N O}_{\mathbf{5}}$ (301.0950): found: 301.0939. 


\section{Synthesis of ethyl 4'-cyano-2-nitro[1,1'-biphenyl]-4-carboxylate (22h)}<smiles>CCOC(=O)c1ccc(-c2ccc(C#N)cc2)c([N+](=O)[O-])c1</smiles>

Prepared according to TP 4 from ethyl 4-iodo-3-nitrobenzoate (1f) (482 mg, $1.50 \mathrm{mmol}$ ), mesitylmagnesium bromide ( $2.3 \mathrm{~mL}, 1.6 \mathrm{mmol}, 0.70 \mathrm{M}$ in THF), $\mathrm{ZnBr}_{2}$ (1.6 mL, $1.6 \mathrm{mmol}$, $1.0 \mathrm{M}$ in THF), $\mathrm{Pd}(\mathrm{dba})_{2}(43.0 \mathrm{mg}, 0.075 \mathrm{mmol}), \mathrm{tfp}(35 \mathrm{mg}, 0.15 \mathrm{mmol})$ and 4-iodoanisole (458 mg, $2.00 \mathrm{mmol})$. Reaction time: $4 \mathrm{~h}$. Purification by flash chromatography (pentane/ethyl acetate $=6: 1$ ) yielded biphenyl $\mathbf{2 2} \mathbf{h}$ as a yellow oil $(235 \mathrm{mg}, 53 \%)$.

mp.: $\quad 143-144{ }^{\circ} \mathrm{C} .{ }^{1} \mathbf{H}-\mathbf{N M R}\left(300 \mathrm{MHz}, \mathrm{CDCl}_{3}, 25{ }^{\circ} \mathrm{C}\right): \delta=8.79\left(\mathrm{~d},{ }^{4} \mathrm{~J}(\mathrm{H}, \mathrm{H})=1.8 \mathrm{~Hz}, 1 \mathrm{H}\right)$, $8.35\left(\mathrm{dd},{ }^{3} J(\mathrm{H}, \mathrm{H})=8.0 \mathrm{~Hz},{ }^{4} J(\mathrm{H}, \mathrm{H})=1.8 \mathrm{~Hz}, 1 \mathrm{H}\right), 7.67-7.64(\mathrm{~m}, 2 \mathrm{H}), 7.53-7.48(\mathrm{~m}, 1 \mathrm{H})$, 7.41-7.35 (m, $2 \mathrm{H}), 4.41\left(\mathrm{q},{ }^{3} J(\mathrm{H}, \mathrm{H})=7.1 \mathrm{~Hz}, 2 \mathrm{H}\right), 1.38\left(\mathrm{t},{ }^{3} J(\mathrm{H}, \mathrm{H})=7.1 \mathrm{~Hz}, 3 \mathrm{H}\right) .{ }^{13} \mathrm{C}-$ NMR $\left(75 \mathrm{MHz}, \mathrm{CDCl}_{3}, 25^{\circ} \mathrm{C}\right): \delta=191.6,162.8,145.8,138.7,134.5,133.7,133.1,132.1$, 128.2, 128.2, 127.9, 124.5, 61.3, 13.3. MS (70 eV, EI), m/z (\%): $296(25)\left[\mathrm{M}^{+}\right], 279(17), 268$ (81), 251 (81), 223 (32), 207 (49), 195 (80), 177 (100), 167 (21), 150 (35). IR (KBr): $\tilde{v}=3435(\mathrm{~s}), 2229(\mathrm{~m}), 1721$ (vs), 1619 (m), $1534(\mathrm{~s}), 139$ (w), 1369 (m), 1354 (m), 1306 (s), 1289 (s), 1241 (m), 1161 (w), 1127 (m), $1111(\mathrm{~m}), 1022$ (w), 1006 (w), 863 (w), 839 (m), $763(\mathrm{~m}), 740(\mathrm{w}), 575(\mathrm{~m}) . \mathbf{C}_{\mathbf{1 6}} \mathbf{H}_{\mathbf{1 2}} \mathbf{N}_{\mathbf{2}} \mathbf{O}_{\mathbf{4}}$ : required: C: 64.86; H: 4.08; N: 9.46; found: $\mathrm{C}$ : 64.50; H: 4.19; N: 9.27.

\section{Synthesis of ethyl 3-nitro-4-(1,3-thiazol-2-yl)benzoate (22i)}

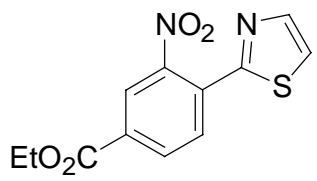

Prepared according to TP 4 from ethyl 4-iodo-3-nitrobenzoate (1f) (321 $\mathrm{mg}, 1.00 \mathrm{mmol}$ ), mesitylmagnesium bromide (1.6 mL, $1.1 \mathrm{mmol}, 0.70 \mathrm{M}$ in THF), $\mathrm{ZnBr}_{2}(1.1 \mathrm{~mL}, 1.1 \mathrm{mmol}$, $1.0 \mathrm{M}$ in THF), $\mathrm{Pd}(\mathrm{dba})_{2}(29 \mathrm{mg}, 0.05 \mathrm{mmol})$, tfp $(23 \mathrm{mg}, 0.10 \mathrm{mmol})$ and 2-bromothiazole (328 mg, $2.00 \mathrm{mmol}$ ). Reaction time: $6 \mathrm{~h}$. Purification by flash chromatography (pentane/diethyl ether $=4: 1$ ) furnished title compound $\mathbf{2 2 i}$ as a yellow solid $(133 \mathrm{mg}, 48 \%$ ).

mp.: $\quad 140.5-143{ }^{\circ} \mathrm{C} .{ }^{1} \mathbf{H}-\mathbf{N M R}\left(400 \mathrm{MHz}, \mathrm{CDCl}_{3}, 25^{\circ} \mathrm{C}\right): \delta=8.40\left(\mathrm{~d},{ }^{4} J(\mathrm{H}, \mathrm{H})=1.7 \mathrm{~Hz}\right.$, $1 \mathrm{H}), 8.25\left(\mathrm{dd},{ }^{3} J(\mathrm{H}, \mathrm{H})=8.1 \mathrm{~Hz},{ }^{4} J(\mathrm{H}, \mathrm{H})=1.7 \mathrm{~Hz}, 1 \mathrm{H}\right), 7.95\left(\mathrm{~d},{ }^{3} J(\mathrm{H}, \mathrm{H})=3.2 \mathrm{~Hz}, 1 \mathrm{H}\right)$, $7.84\left(\mathrm{~d},{ }^{3} J(\mathrm{H}, \mathrm{H})=8.1 \mathrm{~Hz}, 1 \mathrm{H}\right), 7.52\left(\mathrm{~d},{ }^{3} J(\mathrm{H}, \mathrm{H})=3.2 \mathrm{~Hz}, 1 \mathrm{H}\right), 4.44\left(\mathrm{q},{ }^{3} J(\mathrm{H}, \mathrm{H})=7.1 \mathrm{~Hz}\right.$, $2 \mathrm{H}), 1.42\left(\mathrm{t},{ }^{3} \mathrm{~J}(\mathrm{H}, \mathrm{H})=7.1 \mathrm{~Hz}, 3 \mathrm{H}\right) .{ }^{13} \mathbf{C}-\mathbf{N M R}\left(100 \mathrm{MHz}, \mathrm{CDCl}_{3}, 25{ }^{\circ} \mathrm{C}\right): \delta=163.9,160.9$, 148.7, 144.5, 134.1, 132.6, 131.4, 130.7, 125.2, 121.7, 62.1, 14.2. MS (70 eV, EI), m/z (\%): 278 (9) $\left[\mathrm{M}^{+}\right], 233$ (34), 206 (14), 180 (40), 176 (100), 161 (97), 152 (11), 148 (22), 135 (42), 120 (15), 103 (20), 89 (4), 75 (15), 58 (31). IR (KBr): $\widetilde{v}=2963$ (m), 1712 (s), 1546 (s), 1366 
(m), 1288 (m), 1263 (vs), 1238 (m), 1097 (vs), 1020 (vs), 801 (vs), 766 (s). HRMS for $\mathbf{C}_{\mathbf{1 6}} \mathbf{H}_{15} \mathrm{NO}_{5}$ (278.0402): found: 278.0368 .

\section{Synthesis of ethyl 4'-cyano-2'-nitro[1,1'-biphenyl]-4-carboxylate (22j)}<smiles>CCOC(=O)c1ccc(-c2ccc(C#N)cc2[N+](=O)[O-])cc1</smiles>

Prepared according to TP 4 from 4-iodo-3-nitro-benzonitrile (1g) (411 mg, $1.50 \mathrm{mmol}$ ), mesitylmagnesium bromide ( $2.3 \mathrm{~mL}, 1.6 \mathrm{mmol}, 0.70 \mathrm{M}$ in THF), $\mathrm{ZnBr}_{2}$ (1.6 mL, $1.6 \mathrm{mmol}$, $1.0 \mathrm{M}$ in THF), $\mathrm{Pd}(\mathrm{dba})_{2}(39.5 \mathrm{mg}, 0.075 \mathrm{mmol}), \mathrm{tfp}(35 \mathrm{mg}, 0.15 \mathrm{mmol})$ and ethyl 4-iodobenzoate $(621 \mathrm{mg}, 2.50 \mathrm{mmol})$. Purification by flash chromatography (pentane/ethyl acetate $=6: 1$ ) yielded biphenyl $\mathbf{2 2} \mathbf{j}$ as a pale yellow solid (325 $\mathrm{mg}, 73 \%)$.

mp.: $\quad 166-167{ }^{\circ} \mathrm{C} .{ }^{1} \mathbf{H}-\mathbf{N M R}\left(400 \mathrm{MHz}, \mathrm{CDCl}_{3}, 25^{\circ} \mathrm{C}\right): \delta=8.21\left(\mathrm{~d},{ }^{4} J(\mathrm{H}, \mathrm{H})=1.8 \mathrm{~Hz}, 1 \mathrm{H}\right)$, $8.13\left(\mathrm{~d},{ }^{3} J(\mathrm{H}, \mathrm{H})=8.5 \mathrm{~Hz}, 2 \mathrm{H}\right), 7.93\left(\mathrm{dd},{ }^{3} J(\mathrm{H}, \mathrm{H})=8.5 \mathrm{~Hz},{ }^{4} J(\mathrm{H}, \mathrm{H})=1.8 \mathrm{~Hz}, 1 \mathrm{H}\right), 7.60(\mathrm{~d}$, $\left.{ }^{3} J(\mathrm{H}, \mathrm{H})=8.4 \mathrm{~Hz}, 1 \mathrm{H}\right), 7.38\left(\mathrm{~d},{ }^{3} J(\mathrm{H}, \mathrm{H})=8.5 \mathrm{~Hz}, 2 \mathrm{H}\right), 4.40\left(\mathrm{q},{ }^{3} J(\mathrm{H}, \mathrm{H})=7.2 \mathrm{~Hz}, 2 \mathrm{H}\right), 1.41$ $\left(\mathrm{t},{ }^{3} \mathrm{~J}(\mathrm{H}, \mathrm{H})=7.2 \mathrm{~Hz}, 3 \mathrm{H}\right) .{ }^{13} \mathrm{C}-\mathbf{N M R}\left(100 \mathrm{MHz}, \mathrm{CDCl}_{3}, 25{ }^{\circ} \mathrm{C}\right): \delta=165.7,149.1,139.9$, $135.5,132.9,131.4,130.8,130.2,127.9,127.8,116.3,113.2,61.3,14.3$. MS (70 eV, EI), $\mathrm{m} / z$ (\%): 296 (10) [ $\left.\mathrm{M}^{+}\right], 269$ (52), 251 (100), 228 (16), 205 (40), 177 (63), 150 (23), 126 (8), 77 (14), 63 (6), 51 (4). IR (KBr): $\widetilde{v}=3436$ (m), 2235 (m), 1712 (vs), 1610 (w), 1528 (vs), 1358 (s), 1286 (vs), 1110 (m), 849 (m), 761 (w), 712 (w). HRMS for $\mathbf{C}_{\mathbf{1 6}} \mathbf{H}_{\mathbf{1 2}} \mathbf{N}_{\mathbf{2}} \mathbf{O}_{\mathbf{4}}$ (296.0797): found: 296.0779. $\mathbf{C}_{\mathbf{1 6}} \mathbf{H}_{\mathbf{1 2}} \mathbf{N}_{\mathbf{2}} \mathbf{O}_{\mathbf{4}}$ : required: C: 64.86; H: 4.08; N: 9.46; found: C: 64.62, H: 3.64; N: 9.40.

\section{Synthesis of $2,4^{\prime}$-dinitro[1,1'-biphenyl]-4-carbonitrile (22k)}<smiles>N#Cc1ccc(-c2ccc([N+](=O)[O-])cc2)c([N+](=O)[O-])c1</smiles>

Prepared according to TP 4 from 4-iodo-3-nitro-benzonitrile (1g) (411 mg, $1.50 \mathrm{mmol}$ ), mesitylmagnesium bromide $\left(2.3 \mathrm{~mL}, 1.65 \mathrm{mmol}, 0.70 \mathrm{M}\right.$ in THF), $\mathrm{ZnBr}_{2}$ (1.6 mL, $1.6 \mathrm{mmol}$, $1.0 \mathrm{M}$ in THF), $\mathrm{Pd}(\mathrm{dba})_{2}(39.5 \mathrm{mg}, 0.075 \mathrm{mmol}), \mathrm{tfp}(35 \mathrm{mg}, 0.15 \mathrm{mmol})$ and ethyl-4-iodobenzoate $(621 \mathrm{mg}, 2.50 \mathrm{mmol})$. Purification by flash chromatography (pentane/ethyl acetate $=6: 1)$ yielded biphenyl $\mathbf{2 2} \mathbf{k}$ as a pale yellow solid $(325 \mathrm{mg}, 73 \%)$.

mp.: $\quad 142-143{ }^{\circ} \mathrm{C} .{ }^{1} \mathbf{H}-N M R\left(300 \mathrm{MHz}, \mathrm{DMSO}_{6}, 25{ }^{\circ} \mathrm{C}\right): \delta=8.71\left(\mathrm{~d},{ }^{4} J(\mathrm{H}, \mathrm{H})=1.8 \mathrm{~Hz}\right.$, $1 \mathrm{H}), 8.36-8.30(\mathrm{~m}, 3 \mathrm{H}), 7.83\left(\mathrm{~d},{ }^{3} J(\mathrm{H}, \mathrm{H})=8.4 \mathrm{~Hz}, 1 \mathrm{H}\right), 7.71\left(\mathrm{~d},{ }^{3} J(\mathrm{H}, \mathrm{H})=8.9 \mathrm{~Hz}, 2 \mathrm{H}\right)$, $4.40\left(\mathrm{q},{ }^{3} J(\mathrm{H}, \mathrm{H})=7.5 \mathrm{~Hz}, 2 \mathrm{H}\right), 1.41\left(\mathrm{t},{ }^{3} J(\mathrm{H}, \mathrm{H})=7.5 \mathrm{~Hz}, 3 \mathrm{H}\right) .{ }^{13} \mathbf{C}-N M R(75 \mathrm{MHz}$, DMSO$\left.\mathrm{d}_{6}, 25^{\circ} \mathrm{C}\right): \delta=148.7,148.1,142.8,138.1,137.1,133.5,129.8,129.0,124.2,117.0,113.1 . \mathrm{MS}$ 
(70 eV, EI), m/z (\%): 269 (31) [M+], 252 (26), 239 (5), 222 (28), 206 (7), 195 (100), 177 (39), 164 (73 ), 150 (42), 140 (75), 126 (8), 114 (9), 99 (9), 87 (8), 75 (14), 63 (9), 51 (5). IR (KBr): $\tilde{v}=2237(\mathrm{~m}), 1618(\mathrm{w}), 1600(\mathrm{~m}), 1527$ (vs), 1509 (s), 1352 (vs), 1300 (w), 1112 (w), 858 (w), 845 (m), 755 (w), 711 (w). HRMS for $\mathbf{C}_{\mathbf{1 6}} \mathbf{H}_{\mathbf{1 2}} \mathbf{N}_{\mathbf{2}} \mathbf{O}_{\mathbf{4}}$ (269.0437): found: 269.0425. $\mathbf{C}_{\mathbf{1 3}} \mathbf{H}_{7} \mathbf{N}_{\mathbf{3}} \mathbf{O}_{\mathbf{4}}$ : required: C: $58.00, \mathrm{H}: 2.62, \mathrm{~N}: 15.61$; found: C: $57.94, \mathrm{H}: 2.31, \mathrm{~N}$ : 15.39 .

\section{Synthesis of 3'-methoxy-2-nitro[1,1'-biphenyl]-4-carbonitrile (22l)}

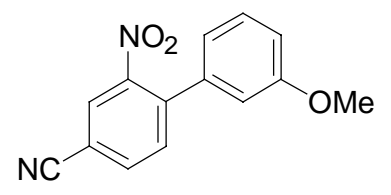

Prepared according to TP 4 from 4-iodo-3-nitrobenzonitrile (1g) (273 mg, $1.00 \mathrm{mmol}$ ), mesitylmagnesium bromide (1.3 mL, $1.1 \mathrm{mmol}, 0.85 \mathrm{M}$ in THF), $\mathrm{ZnBr}_{2}$ (1.1 mL, $1.1 \mathrm{mmol}$, $1.0 \mathrm{M}$ in THF), $\mathrm{Pd}(\mathrm{dba})_{2}(29 \mathrm{mg}, 0.05 \mathrm{mmol}), \mathrm{tfp}(23 \mathrm{mg}, 0.10 \mathrm{mmol})$ and 3 -iodoanisol $(468 \mathrm{mg}, 2.00 \mathrm{mmol})$. Reaction time: $5 \mathrm{~h}$. Purification by flash chromatography (pentane/diethyl ether $=4: 1$ ) yielded biphenyl 221 as a yellow solid $(190 \mathrm{mg}, 75 \%)$.

mp.: $\quad 115-115.5{ }^{\circ} \mathrm{C} .{ }^{1} \mathbf{H}-\mathbf{N M R}\left(300 \mathrm{MHz}, \mathrm{CDCl}_{3}, 25^{\circ} \mathrm{C}\right): \delta=8.10\left(\mathrm{~d},{ }^{4} J(\mathrm{H}, \mathrm{H})=1.8 \mathrm{~Hz}\right.$, $1 \mathrm{H}), 7.87\left(\mathrm{dd},{ }^{3} J(\mathrm{H}, \mathrm{H})=8.0 \mathrm{~Hz},{ }^{4} J(\mathrm{H}, \mathrm{H})=1.8 \mathrm{~Hz}, 1 \mathrm{H}\right), 7.60\left(\mathrm{~d},{ }^{3} J(\mathrm{H}, \mathrm{H})=8.0 \mathrm{~Hz}, 1 \mathrm{H}\right)$, $7.36\left(\mathrm{t},{ }^{3} J(\mathrm{H}, \mathrm{H})=8.0 \mathrm{~Hz}, 1 \mathrm{H}\right), 6.99\left(\mathrm{dd},{ }^{3} J(\mathrm{H}, \mathrm{H})=8.5 \mathrm{~Hz},{ }^{4} J(\mathrm{H}, \mathrm{H})=1.8 \mathrm{~Hz}, 1 \mathrm{H}\right), 6.88-6.82$ $(\mathrm{m}, 2 \mathrm{H}), 3.82$ (s, $3 \mathrm{H}) .{ }^{13} \mathrm{C}-\mathrm{NMR}\left(75 \mathrm{MHz}, \mathrm{CDCl}_{3}, 25^{\circ} \mathrm{C}\right): \delta=159.9,149.4,140.5,136.7$, 135.1, 133.0, 130.2, 127.6, 119.9, 116.5, 114.7, 113.5, 112.5, 55.4. MS (70 eV, EI), m/z (\%): 254 (100) [ $\left.\mathrm{M}^{+}\right], 237$ (11), 226 (40), 209 (32), 195 (46), 183 (31), 177 (30), 164 (90), 153 (44), 140 (36), 127 (17), 114 (9), 101 (9), 87 (8), 75 (11), 63 (12), 55 (15). IR (KBr): $\tilde{v}=2234$ (s), 1604 (m), 1527 (vs), 1442 (w), 1354 (vs), 1238 (vs), 1112 (m), 842 (m), 789 (w), 771 (m), $714(\mathrm{~m}), 632(\mathrm{w})$. HRMS for $\mathbf{C}_{\mathbf{1 4}} \mathbf{H}_{\mathbf{1 0}} \mathbf{N}_{\mathbf{2}} \mathbf{O}_{\mathbf{3}}$ (254.0691): found: 254.0705. $\mathbf{C}_{\mathbf{1 4}} \mathbf{H}_{\mathbf{1 0}} \mathbf{N}_{\mathbf{2}} \mathbf{O}_{\mathbf{3}}$ : required: C: 66.14; H: 3.96; N: 11.02; found: C: 65.97; H: 3.96; N: 11.03.

\section{Synthesis of 1-[(3'-methoxy-2-nitro[1,1'-biphenyl]-4-yl)carbonyl]piperidine (22m)}

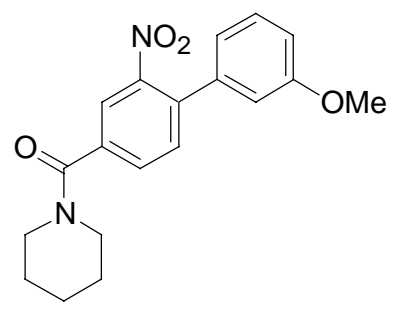

Prepared according to TP 4 from 1-(4-iodo-3-nitrobenzoyl)piperidine (1h) (360 mg, $1.00 \mathrm{mmol})$, mesitylmagnesium bromide (1.3 mL, $1.1 \mathrm{mmol}, 0.85 \mathrm{M}$ in THF), $\mathrm{ZnBr}_{2}(1.1 \mathrm{~mL}$, $1.1 \mathrm{mmol}, 1.0 \mathrm{M}$ in THF), Pd(dba) $2(29 \mathrm{mg}, 0.05 \mathrm{mmol})$, tfp $(23 \mathrm{mg}, 0.10 \mathrm{mmol})$ and $3-$ 
iodoanisole ( $351 \mathrm{mg}, 1.50 \mathrm{mmol}$ ). Reaction time: $4 \mathrm{~h}$. Purification by flash chromatography (pentane/diethyl ether $=1: 1$ ) yielded biphenyl $\mathbf{2 2 m}$ as a light yellow solid (228 $\mathrm{mg}, 67 \%$ ).

mp.: $\quad 90-91{ }^{\circ} \mathrm{C} .{ }^{\mathbf{1}} \mathbf{H}-\mathrm{NMR}\left(300 \mathrm{MHz}, \mathrm{CDCl}_{3}, 25^{\circ} \mathrm{C}\right): \delta=7.86\left(\mathrm{~d},{ }^{4} J(\mathrm{H}, \mathrm{H})=1.8 \mathrm{~Hz}, 2 \mathrm{H}\right)$, $7.61\left(\mathrm{dd},{ }^{3} J(\mathrm{H}, \mathrm{H})=7.8 \mathrm{~Hz},{ }^{4} J(\mathrm{H}, \mathrm{H})=1.8 \mathrm{~Hz}, 1 \mathrm{H}\right), 7.47\left(\mathrm{~d},{ }^{3} J(\mathrm{H}, \mathrm{H})=7.8 \mathrm{~Hz}, 1 \mathrm{H}\right), 7.32(\mathrm{t}$, $\left.{ }^{3} J(\mathrm{H}, \mathrm{H})=7.9 \mathrm{~Hz}, 1 \mathrm{H}\right), 6.95-6.92(\mathrm{~m}, 1 \mathrm{H}), 6.88-6.83(\mathrm{~m}, 2 \mathrm{H}), 3.80$ (s, $\left.3 \mathrm{H}\right), 3.72$ (s_br, $2 \mathrm{H}), 3.40$ (s_br, $2 \mathrm{H}), 1.84-1.50(\mathrm{~m}, 6 \mathrm{H}) .{ }^{13} \mathbf{C}-\mathbf{N M R}\left(75 \mathrm{MHz}, \mathrm{CDCl}_{3}, 25{ }^{\circ} \mathrm{C}\right): \delta=167.3$, 159.7, 149.0, 137.9, 137.0, 126.7, 132.1, 130.5, 129.8, 122.6, 120.1, 114.0, 113.6, 55.3, 48.8, 43.4, 26.5, 25.5, 24.4. MS (70 eV, EI), m/z (\%): 339 (100) [ $\left.\mathrm{M}^{+}\right], 323$ (10), $306(13), 294$ (21), 256 (54), 228 (10), 209 m(44), 180 (19), 152 (19), 139 (27), 127 (4), 84 (5). IR $(\mathrm{KBr}): \tilde{v}=2857(\mathrm{~m}), 1631(\mathrm{vs}), 1601$ (s), $1530(\mathrm{~s}), 1445$ (s), 1350 (s), $1321(\mathrm{~m}), 1286(\mathrm{~s})$, 1260 (m), 1220 (s), 1179 (m), 1022 (m), 855 (m), 847 (m), 795 (m). HRMS for $\mathbf{C}_{\mathbf{1 9}} \mathbf{H}_{\mathbf{2 0}} \mathbf{N}_{\mathbf{2}} \mathbf{O}_{\mathbf{4}}$ (340.1423): found: 340.1428 .

\section{Synthesis of 1-[(2,4'-dinitro[1,1'-biphenyl]-4-yl)carbonyl]piperidine (22n)}

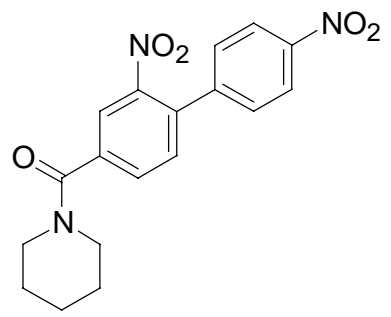

Prepared according to TP 4 from 1-(4-iodo-3-nitrobenzoyl)piperidine (1h) (360 mg, $1.00 \mathrm{mmol})$, mesitylmagnesium bromide (1.3 mL, $1.1 \mathrm{mmol}, 0.85 \mathrm{M}$ in THF), $\mathrm{ZnBr}_{2}(1.1 \mathrm{~mL}$, $1.1 \mathrm{mmol}, 1.0 \mathrm{M}$ in THF), Pd(dba) 2 (29 mg, $0.05 \mathrm{mmol})$, tfp $(23 \mathrm{mg}, 0.10 \mathrm{mmol})$ and $4-$ iodonitrobenzene $(498 \mathrm{mg}, 2.00 \mathrm{mmol})$. Reaction time: $3 \mathrm{~h}$. Purification by flash chromatography (pentane/diethyl ether $=2: 1$ ) yielded biphenyl 22n as a pale yellow solid (345 mg, 77\%).

mp.: $\quad 152.5-153{ }^{\circ} \mathrm{C} .{ }^{1} \mathbf{H}-\mathbf{N M R}\left(300 \mathrm{MHz}, \mathrm{CDCl}_{3}, 25^{\circ} \mathrm{C}\right): \delta=8.27\left(\mathrm{~d},{ }^{3} J(\mathrm{H}, \mathrm{H})=8.4 \mathrm{~Hz}\right.$, $2 \mathrm{H}), 8.01\left(\mathrm{~d},{ }^{4} J(\mathrm{H}, \mathrm{H})=1.8 \mathrm{~Hz}, 1 \mathrm{H}\right), 7.70\left(\mathrm{dd},{ }^{3} J(\mathrm{H}, \mathrm{H})=7.9 \mathrm{~Hz},{ }^{4} J(\mathrm{H}, \mathrm{H})=1.8 \mathrm{~Hz}, 1 \mathrm{H}\right)$, 7.49-7.44 (m, $3 \mathrm{H}), 3.72$ (s_br, $2 \mathrm{H}$ ), 3.40 (s_br, $2 \mathrm{H}$ ), 1.75-1.52 (m, $6 \mathrm{H}) .{ }^{13} \mathrm{C}-\mathbf{N M R}$ (75 MHz, $\mathrm{CDCl}_{3}, 25{ }^{\circ} \mathrm{C}$ ): $\delta=166.8,148.3,147.8,143.6,138.1,135.2,131.9,131.1,128.9$, 123.9, 123.3, 48.9, 43.3, 26.5, 25.4, 24.3. MS (70 eV, EI), m/z (\%): 354 (100) $[\mathrm{M}-\mathrm{H}]^{+}, 338$ (19), 320 (9), 309 (17), 271 (54), 225 (26), 179 (47), 167 (3), 150 (28), 139 (11), 84 (8). IR $(\mathrm{KBr}): \widetilde{v}=2940(\mathrm{w}), 1632$ (vs), 1599 (m), 1523 (vs), 1466 (w), 1444 (s), 1348 (vs), 1284 (m), 1113 (w), 1006 (w), 911 (w), 854 (m), 847 (m), 744 (m), 694 (w). HRMS for $\mathbf{C}_{\mathbf{1 8}} \mathbf{H}_{17} \mathbf{N}_{3} \mathbf{O}_{\mathbf{5}}$ (355.1168): found: 355.1162. $\mathbf{C}_{\mathbf{1 8}} \mathbf{H}_{17} \mathbf{N}_{\mathbf{3}} \mathbf{O}_{5}$ : required: $\mathrm{C}: 60.84 ; \mathrm{H}: 4.82 ; \mathrm{N}$ : 11.83; found: C: 60.47 ; H: 4.87; N: 11.71. 


\section{Synthesis of compounds 26}

\section{Synthesis of 6-nitro-3-phenyl-2-benzofuran-1(3H)-one (26a)}

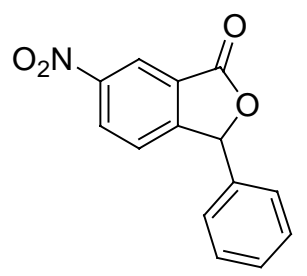

Prepared according to TP 5 from ethyl 2-iodo-5-nitrobenzoate (1i) (321 mg, $1 \mathrm{mmol}$ ), $\mathrm{PhMgCl}(0.55 \mathrm{~mL}, 1.1 \mathrm{mmol}, 2.0 \mathrm{M}$ in THF) and benzaldehyde (212 mg, $2 \mathrm{mmol})$. Reaction time: $2 \mathrm{~h}$ at $-78{ }^{\circ} \mathrm{C}$. Purification by flash chromatography (pentane/diethyl ether $=9: 1$ ) furnished title compound 26a as a pale yellow oil (199 $\mathrm{mg}, 78 \%$ ).

${ }^{1} \mathbf{H}$-NMR $\left(300 \mathrm{MHz}, \mathrm{CDCl}_{3}, 25{ }^{\circ} \mathrm{C}\right): \delta=8.75\left(\mathrm{~d},{ }^{4} J(\mathrm{H}, \mathrm{H})=2.2 \mathrm{~Hz}, 1 \mathrm{H}\right), 8.51$ (dd, $\left.{ }^{3} J(\mathrm{H}, \mathrm{H})=8.4 \mathrm{~Hz},{ }^{4} J(\mathrm{H}, \mathrm{H})=2.2 \mathrm{~Hz}, 1 \mathrm{H}\right), 7.56\left(\mathrm{~d},{ }^{3} J(\mathrm{H}, \mathrm{H})=8.4 \mathrm{~Hz}, 1 \mathrm{H}\right), 7.45-7.37(\mathrm{~m}, 5 \mathrm{H})$, 6.53 (s, $1 \mathrm{H}) .{ }^{13} \mathrm{C}-\mathrm{NMR}\left(75 \mathrm{MHz}, \mathrm{CDCl}_{3}, 25{ }^{\circ} \mathrm{C}\right): \delta=167.9,154.7,149.0,134.6,129.7$, 129.1, 129.0, 127.1, 126.8, 124.4, 121.0, 82.6. MS (70 eV, EI), m/z (\%): 255 (100) $\left[\mathrm{M}^{+}\right], 226$ (4), 180 (12), 165 (28), 149 (40), 139 (8), 132 (9), 105 (85), 87 (3), 77 (23). IR (KBr): $\tilde{v}=1778$ (vs), 1615 (m), 1537 (vs), 1455 (m), 1347 (vs), 1303 (m), 1254 (m), 1097 (vs), 797 (w), 763 (w), 739 (m), 700 (m), 620 (w). HRMS for $\mathbf{C}_{\mathbf{1 4}} \mathbf{H}_{\mathbf{9}} \mathbf{N O}_{\mathbf{4}}$ (255.0532): found: 255.0504.

\section{Synthesis of ethyl 2-allyl-5-nitrobenzoate (26b)}<smiles>C=CCc1ccc([N+](=O)[O-])cc1C(=O)OCC</smiles>

Prepared according to TP 5 from ethyl 2-iodo-5-nitrobenzoate (1i) (321 mg, $1.00 \mathrm{mmol}$ ), $\mathrm{PhMgCl}$ (0.66 mL, $1.1 \mathrm{mmol}, 1.6 \mathrm{M}$ in THF), CuCN.2LiCl (1.1 mL, $1.1 \mathrm{mmol}, 1.0 \mathrm{M}$ in THF) and allyl bromide $(240 \mathrm{mg}, 2.00 \mathrm{mmol})$. Reaction time: $2 \mathrm{~h}$ at $-78^{\circ} \mathrm{C}$. Purification by flash chromatography (pentane/diethyl ether $=29: 1$ ) furnished title compound $\mathbf{2 6} \mathbf{b}$ as a yellow oil (204 mg, 87\%).

${ }^{1}$ H-NMR $\left(300 \mathrm{MHz}, \mathrm{CDCl}_{3}, 25^{\circ} \mathrm{C}\right): \delta=8.70 \quad\left(\mathrm{~d},{ }^{4} J(\mathrm{H}, \mathrm{H})=2.7 \mathrm{~Hz}, 1 \mathrm{H}\right), 8.24 \quad(\mathrm{dd}$, $\left.{ }^{3} J(\mathrm{H}, \mathrm{H})=8.9 \mathrm{~Hz},{ }^{4} J(\mathrm{H}, \mathrm{H})=2.7 \mathrm{~Hz}, 1 \mathrm{H}\right), 7.46\left(\mathrm{~d},{ }^{3} J(\mathrm{H}, \mathrm{H})=8.9 \mathrm{~Hz}, 1 \mathrm{H}\right), 6.03-5.89(\mathrm{~m}, 1 \mathrm{H})$, 5.12-5.00 (m, $2 \mathrm{H}), 4.39\left(\mathrm{q},{ }^{3} J(\mathrm{H}, \mathrm{H})=7.1 \mathrm{~Hz}, 2 \mathrm{H}\right), 3.84\left(\mathrm{~d},{ }^{3} J(\mathrm{H}, \mathrm{H})=6.6 \mathrm{~Hz}, 2 \mathrm{H}\right), 1.40(\mathrm{t}$, $\left.{ }^{3} J(\mathrm{H}, \mathrm{H})=7.1 \mathrm{~Hz}, 3 \mathrm{H}\right) .{ }^{13} \mathbf{C}-\mathbf{N M R}\left(75 \mathrm{MHz}, \mathrm{CDCl}_{3}, 25{ }^{\circ} \mathrm{C}\right): \delta=165.5,148.9,146.2,135.6$, 132.0, 131.2, 126.1, 125.7, 117.1, 61.8, 38.2, 14.2. MS (70 eV, EI), $m / z(\%): 235$ (23) $\left[\mathrm{M}^{+}\right]$, 220 (35), 207 (34), 192 (100), 179 (11), 160 (12), 144 (8), 115 (42), 89 (5). IR (KBr): $\tilde{v}=2983(\mathrm{w}), 1727$ (vs), 1613 (m), 1525 (vs), 1479 (m), 1445 (m), 1350 (vs), 1252 (s), 1127 (s), 1067 (m), 1018 (m), 921 (m), 837 (w), 745 (w), 716 (w), 604 (w). HRMS for $\mathbf{C}_{12} \mathbf{H}_{13} \mathbf{N O}_{4}$ (235.0845): found: 235.0845 . 


\section{Synthesis of ethyl 2-benzoyl-5-nitrobenzoate (26c)}<smiles>CCOC(=O)c1cc([N+](=O)[O-])ccc1C(=O)c1ccccc1</smiles>

Prepared according to TP 5 from ethyl 2-iodo-5-nitrobenzoate (1i) (642 mg, $2 \mathrm{mmol}$ ), $\mathrm{PhMgCl}$ (1.1 mL, $2.2 \mathrm{mmol}, 2.0 \mathrm{M}$ in THF), CuCN.2LiCl (2.2 mL, $2.2 \mathrm{mmol}, 1.0 \mathrm{M}$ in THF) and benzoyl bromide $(648 \mathrm{mg}, 3.50 \mathrm{mmol})$. Reaction time: $1 \mathrm{~h}$ at $-78{ }^{\circ} \mathrm{C}$. Purification by flash chromatography (pentane/diethyl ether $=9: 1$ ) furnished title compound $\mathbf{2 6 c}$ as a yellow solid (570 mg, 94\%).

mp.: $\quad 88-89.5{ }^{\circ} \mathrm{C} .{ }^{1} \mathbf{H}-\mathbf{N M R}\left(300 \mathrm{MHz}, \mathrm{CDCl}_{3}, 25^{\circ} \mathrm{C}\right): \delta=8.83\left(\mathrm{~d},{ }^{4} J(\mathrm{H}, \mathrm{H})=2.8 \mathrm{~Hz}, 1 \mathrm{H}\right)$, $8.40\left(\mathrm{dd},{ }^{3} \mathrm{~J}(\mathrm{H}, \mathrm{H})=8.8 \mathrm{~Hz},{ }^{4} J(\mathrm{H}, \mathrm{H})=2.8 \mathrm{~Hz}, 1 \mathrm{H}\right), 7.67-7.64(\mathrm{~m}, 2 \mathrm{H}), 7.53-7.51(\mathrm{~m}, 1 \mathrm{H})$, 7.51-7.49 (m, $2 \mathrm{H}), 7.42-7.36(\mathrm{~m}, 1 \mathrm{H}), 4.10\left(\mathrm{q},{ }^{3} J(\mathrm{H}, \mathrm{H})=7.1 \mathrm{~Hz}, 2 \mathrm{H}\right), 1.07-1.03(\mathrm{~m}$, $\left.{ }^{3} J(\mathrm{H}, \mathrm{H})=7.1 \mathrm{~Hz}, 3 \mathrm{H}\right) .{ }^{13} \mathbf{C}-\mathbf{N M R}\left(75 \mathrm{MHz}, \mathrm{CDCl}_{3}, 25{ }^{\circ} \mathrm{C}\right): \delta=194.8,163.7,148.2,147.3$, 136.1, 133.9, 130.9, 129.3, 128.9, 128.8, 126.9, 125.4, 62.4, 13.6. MS (70 eV, EI), m/z (\%): 299 (22) [M+], 270 (4), 254 (14), 222 (15), 194 (51), 180 (3), 152 (7), 105 (100), 77 (28). IR (KBr): $\tilde{v}=1723$ (vs), 1672 (vs), 1606 (s), $1596(\mathrm{~s}), 1582$ (m), 1528 (vs), 1450 (s), 1367 (m), 1353 (vs), 1313 (vs), 1278 (vs), 1250 (s), 1017 (m), 908 (m), 863 (w), 848 (m), 838 (w), 792 (w), 742 (m), 714 (s), 698 (s), 690 (m). HRMS for $\mathbf{C}_{\mathbf{1 6}} \mathbf{H}_{\mathbf{1 3}} \mathbf{N O}_{\mathbf{5}}$ (299.0794): found: 299.0801. $\mathbf{C}_{16} \mathbf{H}_{13} N_{O_{5}}$ : required: C: 64.21; H: 4.38; N: 4.68; found: C: 63.98; H: 4.76; N: 4.67.

\section{Synthesis of ethyl 2-[2-(ethoxycarbonyl)-2-propenyl]-5-nitrobenzoate (26d)}<smiles>C=C(Cc1ccc([N+](=O)[O-])cc1C(=O)OCC)C(=O)OCC</smiles>

Prepared according to TP 5 from ethyl 2-iodo-5-nitrobenzoate (1i) (642 $\mathrm{mg}, 2.00 \mathrm{mmol}$ ), $\mathrm{PhMgCl}$ (1.1 mL, $2.2 \mathrm{mmol}, 2.0 \mathrm{M}$ in THF), CuCN.2LiCl (2.2 mL, $2.2 \mathrm{mmol}, 1.0 \mathrm{M}$ in THF) and ethyl (2-bromomethyl) acrylate $(580 \mathrm{mg}, 3.00 \mathrm{mmol})$. Reaction time: $1 \mathrm{~h}$ at $-78{ }^{\circ} \mathrm{C}$. Purification by flash chromatography (pentane/diethyl ether $=4: 1$ ) furnished title compound 26d as an orange oil (411 mg, 67\%).

${ }^{1}$ H-NMR $\left(300 \mathrm{MHz}, \mathrm{CDCl}_{3}, 25^{\circ} \mathrm{C}\right): \delta=8.66(\mathrm{~d}, 1 \mathrm{H}), 8.19\left(\mathrm{dd},{ }^{4} J(\mathrm{H}, \mathrm{H})=2.7 \mathrm{~Hz}\right.$, $\left.{ }^{3} J(\mathrm{H}, \mathrm{H})=8.6 \mathrm{~Hz}, 1 \mathrm{H}\right), 7.40(\mathrm{~d}, 1 \mathrm{H}), 6.20(\mathrm{~d}, 1 \mathrm{H}), 5.29-5.28(\mathrm{~m}, 1 \mathrm{H}), 4.30(\mathrm{q}$, $\left.{ }^{3} J(\mathrm{H}, \mathrm{H})=7.1 \mathrm{~Hz}, 2 \mathrm{H}\right), 4.15-4.11(\mathrm{~m}, 2 \mathrm{H}), 4.08-4.06(\mathrm{~m}, 2 \mathrm{H}), 1.33\left(\mathrm{t},{ }^{3} J(\mathrm{H}, \mathrm{H})=7.1 \mathrm{~Hz}\right.$, $3 \mathrm{H}), 1.21-1.13$ (m, $3 \mathrm{H}) .{ }^{13} \mathbf{C}-\mathbf{N M R}\left(75 \mathrm{MHz}, \mathrm{CDCl}_{3}, 25^{\circ} \mathrm{C}\right): \delta=165.4,164.4,146.7,145.4$, $137.9,131.4,130.6,125.8,125.0,124.8,60.8,60.0,34.8,13.2,13.1$. MS $(70 \mathrm{eV}, \mathrm{EI}), \mathrm{m} / \mathrm{z}$ 


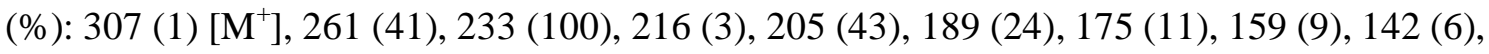
131 (12), 115 (29), 103 (4), 89 (3), 77 (4). IR (KBr): $\tilde{v}=1722$ (vs), 1526 (vs), 1367 (s), 1351 (vs), 1254 (vs), 1137 (s), 1070 (s), 1023 (m), 935 (w), 921 (w), 838 (w), 821 (w), 787 (w), 738 (w), 722 (w). HRMS for $\mathbf{C}_{\mathbf{1 5}} \mathbf{H}_{\mathbf{1 7}} \mathbf{N O}_{\mathbf{6}}$ (307.1056): found: 307.1058.

\section{Synthesis of 2-benzoyl- $N, N$-diethyl-5-nitrobenzamide (26e)}

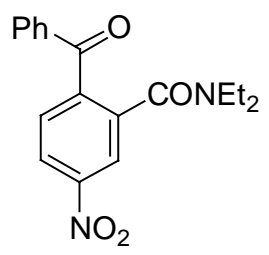

Prepared according to TP 5 from $N, N$-diethyl-2-iodo-5-nitrobenzamide (1j) (694 mg, $2.00 \mathrm{mmol}), \mathrm{PhMgCl}(1.1 \mathrm{~mL}, 2.2 \mathrm{mmol}, 2.0 \mathrm{M}$ in THF), CuCN.2LiCl (2.2 mL, $2.2 \mathrm{mmol}$, $1.0 \mathrm{M}$ in THF) and benzoyl bromide $(648 \mathrm{mg}, 3.50 \mathrm{mmol})$. Reaction time: $1 \mathrm{~h}$ at $-78{ }^{\circ} \mathrm{C}$. Purification by flash chromatography (pentane/ethyl acetate $=9: 1$ ) furnished title compound 26e as a yellow oil (313 $\mathrm{mg}, 68 \%)$.

${ }^{1}$ H-NMR $\left(300 \mathrm{MHz}, \mathrm{CDCl}_{3}, 25^{\circ} \mathrm{C}\right): \delta=8.28\left(\mathrm{dd},{ }^{3} J(\mathrm{H}, \mathrm{H})=8.4 \mathrm{~Hz},{ }^{4} J(\mathrm{H}, \mathrm{H})=2.2 \mathrm{~Hz}, 1 \mathrm{H}\right)$, 8.24-8.21 (m, $1 \mathrm{H}), 7.77-7.72(\mathrm{~m}, 2 \mathrm{H}), 7.64\left(\mathrm{~d},{ }^{3} J(\mathrm{H}, \mathrm{H})=8.4 \mathrm{~Hz}, 1 \mathrm{H}\right), 7.62-7.56(\mathrm{~m}, 1 \mathrm{H})$, 7.47-7.41 (m, $2 \mathrm{H}), 3.40\left(\mathrm{q},{ }^{3} J(\mathrm{H}, \mathrm{H})=7.1 \mathrm{~Hz}, 2 \mathrm{H}\right), 3.25\left(\mathrm{q},{ }^{3} J(\mathrm{H}, \mathrm{H})=7.1 \mathrm{~Hz}, 2 \mathrm{H}\right), 1.15(\mathrm{t}$, $\left.{ }^{3} J(\mathrm{H}, \mathrm{H})=7.1 \mathrm{~Hz}, 3 \mathrm{H}\right), 1.02\left(\mathrm{t},{ }^{3} \mathrm{~J}(\mathrm{H}, \mathrm{H})=7.1 \mathrm{~Hz}, 2 \mathrm{H}\right) .{ }^{13} \mathbf{C}-\mathbf{N M R}\left(75 \mathrm{MHz}, \mathrm{CDCl}_{3}, 25{ }^{\circ} \mathrm{C}\right)$ : $\delta=194.9,167.3,148.4,143.0,139.5,136.0,134.0,130.3,129.2,128.6,123.2,121.7,43.0$, 39.2, 13.7, 12.1. MS (70 eV, EI), m/z (\%): 325 (1) [M] $]^{+}, 254$ (73), 238 (3), 208 (29), 196 (6), 180 (21), 152 (43), 105 (4), 72 (100). IR (KBr): $\tilde{v}=1671$ (s), 1634 (vs), 1528 (vs), 1448 (s), 1349 (vs), 1316 (s), 1278 (vs), 1218 (m), 1113 (m), 940 (m), 901 (m), 851 (m), 733 (m), 705 (m). HRMS for $\mathbf{C}_{\mathbf{1 8}} \mathbf{H}_{\mathbf{1 8}} \mathbf{N}_{\mathbf{2}} \mathbf{O}_{\mathbf{4}}$ (326.1267): found: 326.1277 .

\section{Synthesis of compounds 27}

\section{Synthesis of 2-[hydroxy(phenyl)methyl]-4-nitrophenyl 4-methylbenzenesulfonate} (27a)

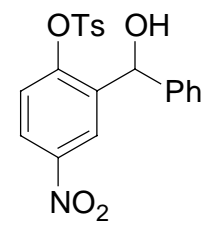

Prepared according to TP 5 from 2-iodo-4-nitrophenyl 4-methylbenzenesulfonate (1q) (439 mg, $1.00 \mathrm{mmol}), \mathrm{PhMgCl}(0.65 \mathrm{~mL}, 1.1 \mathrm{mmol}, 1.6 \mathrm{M}$ in THF) and benzaldehyde 
$(127 \mathrm{mg}, 1.20 \mathrm{mmol})$. Purification by flash chromatography (pentane/diethyl ether $=4: 1$ ) furnished title compound 27a as a colourless oil (320mg, 77\%).

${ }^{1} \mathbf{H}-\mathbf{N M R}\left(300 \mathrm{MHz}, \mathrm{CDCl}_{3}, 25^{\circ} \mathrm{C}\right): \delta=8.27\left(\mathrm{~d},{ }^{4} J(\mathrm{H}, \mathrm{H})=2.7 \mathrm{~Hz}, 1 \mathrm{H}\right), 8.06(\mathrm{dd}$, $\left.{ }^{3} J(\mathrm{H}, \mathrm{H})=8.9 \mathrm{~Hz},{ }^{4} J(\mathrm{H}, \mathrm{H})=2.7 \mathrm{~Hz}, 1 \mathrm{H}\right), 7.70\left(\mathrm{~d},{ }^{3} J(\mathrm{H}, \mathrm{H})=8.4 \mathrm{~Hz}, 2 \mathrm{H}\right), 7.36-7.26(\mathrm{~m}, 7 \mathrm{H})$, $7.21\left(\mathrm{~d},{ }^{3} J(\mathrm{H}, \mathrm{H})=8.9 \mathrm{~Hz}, 1 \mathrm{H}\right), 6.01(\mathrm{~s}, 1 \mathrm{H}), 2.80$ (s_br, $\left.1 \mathrm{OH}\right), 2.47$ (s, $\left.3 \mathrm{H}\right) .{ }^{13} \mathbf{C}-\mathbf{N M R}$ $\left(75 \mathrm{MHz}, \mathrm{CDCl}_{3}, 25{ }^{\circ} \mathrm{C}\right): \delta=150.7,146.5,146.2,141.1,138.9,131.8,130.2,128.6,128.3$, $128.1,126.5,124.0,123.8,122.1,69.6,21.7 . \mathrm{MS}(70 \mathrm{eV}, \mathrm{EI}), \mathrm{m} / z(\%): 399(0.1)\left[\mathrm{M}^{+}\right], 382$ (14), 319 (23), 301 (13), 244 (100), 226 (34), 180 (11), 166 (22), 155 (10), 91 (61), 77 (13). IR (KBr): $\tilde{v}=3541(\mathrm{w}), 1596(\mathrm{~m}), 1530$ (vs), 1475 (m), 1381 (s), 1350 (vs), 1209 (s), 1178 (vs), 1090 (s), 1038 (m), 856 (s), 766 (m), 717 (s), 700 (s), 670 (m), 565 (m), 550 (m). HRMS for $\mathbf{C}_{20} \mathbf{H}_{17} \mathbf{N O} \mathbf{O}_{6} \mathbf{S}$ (399.0777): found: 399.0812 .

\section{Synthesis of 2-allyl-4-nitrophenyl 4-methylbenzenesulfonate (27b)}

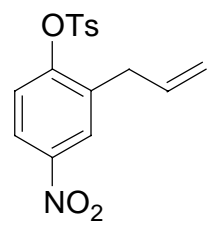

Prepared according to TP 5 form 2-iodo-4-nitrophenyl 4-methylbenzenesulfonate (1q) (439 mg, $1.00 \mathrm{mmol}), \mathrm{PhMgCl}(0.65 \mathrm{~mL}, 1.1 \mathrm{mmol}, 1.6 \mathrm{M}$ in THF), CuCN.2LiCl (1.1 mL, $1.1 \mathrm{mmol}, 1.0 \mathrm{M}$ in THF) and allyl bromide $(0.17 \mathrm{~mL}, 1.6 \mathrm{mmol})$. Purification by flash chromatography (pentane/diethyl ether $=19: 1$ ) furnished title compound $\mathbf{2 7} \mathbf{b}$ as a colourless oil (311mg, 93\%).

${ }^{1}$ H-NMR $\left(300 \mathrm{MHz}, \mathrm{CDCl}_{3}, 25{ }^{\circ} \mathrm{C}\right): \delta=8.08-8.02(\mathrm{~m}, 2 \mathrm{H}), 7.77\left(\mathrm{~d},{ }^{3} J(\mathrm{H}, \mathrm{H})=8.4 \mathrm{~Hz}, 2 \mathrm{H}\right)$, 7.38-7.35 (m, $1 \mathrm{H}), 7.27\left(\mathrm{~d},{ }^{3} J(\mathrm{H}, \mathrm{H})=8.9 \mathrm{~Hz}, 1 \mathrm{H}\right), 5.81-5.67(\mathrm{~m}, 1 \mathrm{H}), 5.13$ (ddt, $\left.{ }^{3} J(\mathrm{H}, \mathrm{H})=9.7 \mathrm{~Hz},{ }^{2} J(\mathrm{H}, \mathrm{H}),{ }^{4} J(\mathrm{H}, \mathrm{H})=1.8 \mathrm{~Hz}, 1 \mathrm{H}\right), 5.04\left(\mathrm{ddt},{ }^{3} J(\mathrm{H}, \mathrm{H})=16.8 \mathrm{~Hz},{ }^{2} J(\mathrm{H}, \mathrm{H})\right.$, $\left.{ }^{4} J(\mathrm{H}, \mathrm{H})=1.8 \mathrm{~Hz}, 1 \mathrm{H}\right), 3.27\left(\mathrm{~d},{ }^{3} J(\mathrm{H}, \mathrm{H})=6.6 \mathrm{~Hz}, 1 \mathrm{H}\right), 2.47(\mathrm{~s}, 3 \mathrm{H}) .{ }^{13} \mathrm{C}-\mathbf{N M R}(75 \mathrm{MHz}$, $\left.\mathrm{CDCl}_{3}, 25^{\circ} \mathrm{C}\right): \delta=152.0,146.2,146.0,135.4,133.7,132.4,130.1,128.4,125.9,122.9$, 118.4, 112.6, 33.8, 21.7. MS (70 eV, EI), $m / z(\%): 334$ (17) [M+H] $]^{+}, 317$ (19), 178 (48), 155 (68), 132 (12), 103 (7), 91 (100), 77 (4), 65 (8). IR (KBr): $\tilde{v}=3081(\mathrm{w}), 1596(\mathrm{w}), 1585$ (w), 1528 (vs), 1479 (m), 1381 (vs), 1350 (vs), 1218 (s), 1191 (vs), 1179 (vs), 1159 (s), 1091 (s), 1074 (s), 922 (m), 850 (vs), 809 (s), 761 (s), 735 (s), 708 (s), 669 (m), 577 (m), 550 (m). HRMS for $\mathbf{C}_{16} \mathbf{H}_{15} \mathbf{N O}_{5} \mathbf{S}$ (333.0671): found: $334.0751[\mathrm{M}+\mathrm{H}]^{+}$.

\section{Synthesis of 2-[hydroxy(phenyl)methyl]-6-iodo-4-nitrophenyl 4- methylbenzenesulfonate (27c)}

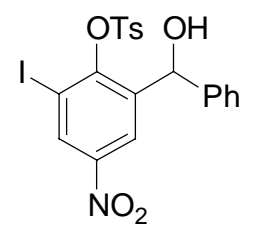


Prepared according to TP 5 form 2,6-diiodo-4-nitrophenyl 4-methylbenzenesulfonate (1r) (529 mg, $1.00 \mathrm{mmol}), \mathrm{PhMgCl}(0.65 \mathrm{~mL}, 1.1 \mathrm{mmol}, 1.6 \mathrm{M}$ in THF) and benzaldehyde $(127 \mathrm{mg}, 1.20 \mathrm{mmol})$. Purification by flash chromatography (pentane/diethyl ether $=4: 1$ ) furnished title compound 27c as a light yellow solid (445 mg, 85\%).

mp.: $\quad 156-158{ }^{\circ} \mathrm{C} .{ }^{\mathbf{1}} \mathbf{H}-\mathbf{N M R}\left(300 \mathrm{MHz}, \mathrm{CDCl}_{3}, 25{ }^{\circ} \mathrm{C}\right): \delta=8.51\left(\mathrm{~d},{ }^{3} J(\mathrm{H}, \mathrm{H})=2.8 \mathrm{~Hz}, 1 \mathrm{H}\right)$, $8.37\left(\mathrm{~d},{ }^{3} J(\mathrm{H}, \mathrm{H})=2.8 \mathrm{~Hz}, 1 \mathrm{H}\right), 7.94\left(\mathrm{~d},{ }^{3} J(\mathrm{H}, \mathrm{H})=8.4 \mathrm{~Hz}, 2 \mathrm{H}\right), 7.43\left(\mathrm{~d},{ }^{3} J(\mathrm{H}, \mathrm{H})=8.4 \mathrm{~Hz}\right.$, $2 \mathrm{H}), 7.38-7.27$ (m, $5 \mathrm{H}), 6.42$ (s_br, $1 \mathrm{H}), 3.12$ (s_br, $1 \mathrm{OH}), 2.50$ (s, $3 \mathrm{H}) .{ }^{13} \mathbf{C}-\mathbf{N M R}$ $\left(75 \mathrm{MHz}, \mathrm{CDCl}_{3}, 25{ }^{\circ} \mathrm{C}\right): \delta=151.5,146.8,146.6,142.3,141.3,134.3,133.0,130.2,129.0$,

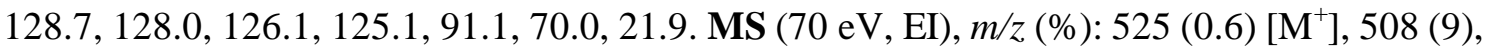
443 (15), 428 (35), 415 (29), 370 (100), 369 (14), 352 (52), 321 (9), 305 (9), 292 (10), 155 (14), 139 (18), 105 (29), 91 (81), 77 (28). IR (KBr): $\tilde{v}=3436$ (m), 1526 (vs), 1367 (s), 1352 (vs), 1179 (s), 1088 (s), 846 (m), 728 (vs), 701 (m), 570 (m). HRMS for $\mathbf{C}_{\mathbf{2 0}} \mathbf{H}_{16} \mathbf{I N O} \mathbf{N} \mathbf{S}$ (524.9743): found: 524.9745.

\section{Synthesis of 2-allyl-6-iodo-4-nitrophenyl 4-methylbenzenesulfonate (27d)}

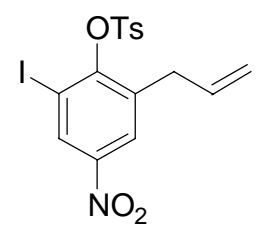

Prepared according to TP 5 form 2,6-diiodo-4-nitrophenyl 4-methylbenzenesulfonate (1r) (545 mg, $1.00 \mathrm{mmol}), \mathrm{PhMgCl}(0.65 \mathrm{~mL}, 1.1 \mathrm{mmol}, 1.6 \mathrm{M}$ in THF), CuCN.2LiCl (1.1 mL, $1.1 \mathrm{mmol}, 1.0 \mathrm{M}$ in THF) and allyl bromide $(0.17 \mathrm{~mL}, 1.6 \mathrm{mmol})$. Purification by flash chromatography (pentane/diethyl ether $=19: 1$ ) furnished title compound $\mathbf{2 7 d}$ as a colourless oil (401 mg, 87\%).

${ }^{1} \mathbf{H}$-NMR $\left(300 \mathrm{MHz}, \quad \mathrm{CDCl}_{3}, \quad 25{ }^{\circ} \mathrm{C}\right): \delta=8.50 \quad\left(\mathrm{~d},{ }^{4} J(\mathrm{H}, \mathrm{H})=2.8 \mathrm{~Hz}, 1 \mathrm{H}\right), 8.13(\mathrm{~d}$, $\left.{ }^{4} J(\mathrm{H}, \mathrm{H})=2.8 \mathrm{~Hz}, 1 \mathrm{H}\right), 7.92\left(\mathrm{~d},{ }^{3} J(\mathrm{H}, \mathrm{H})=8.4 \mathrm{~Hz}, 2 \mathrm{H}\right), 7.40\left(\mathrm{~d},{ }^{3} J(\mathrm{H}, \mathrm{H})=8.4 \mathrm{~Hz}, 2 \mathrm{H}\right), 5.92-$ $5.79(\mathrm{~m}, 1 \mathrm{H}), 5.25-5.16(\mathrm{~m}, 2 \mathrm{H}), 3.60\left(\mathrm{~d},{ }^{3} J(\mathrm{H}, \mathrm{H})=6.6 \mathrm{~Hz}, 1 \mathrm{H}\right), 2.49(\mathrm{~s}, 3 \mathrm{H}) .{ }^{13} \mathbf{C}-\mathbf{N M R}$ $\left(75 \mathrm{MHz}, \mathrm{CDCl}_{3}, 25^{\circ} \mathrm{C}\right): \delta=153.4,146.4,146.1,137.9,134.0,133.8,133.3,130.1,128.4$,

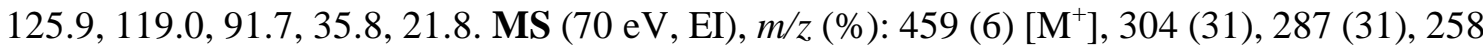
(9), 177 (1), 155 (100), 139 (4), 91 (99), 77 (11), 65 (14). IR (KBr): $\tilde{v}=3081(\mathrm{w}), 1596(\mathrm{w})$, 1528 (vs), 1425 (m), 1369 (s), 1345 (vs), 1311 (w), 1238 (w), 1200 (s), 1181 (s), 1178 (vs), 1145 (w), 1090 (m), 1076 (s), 924 (m), 841 (vs), 814 (w), 759 (s), 730 (vs), 669 (s), 573 (m), 545 (m). HRMS for $\mathbf{C}_{\mathbf{1 6}} \mathbf{H}_{\mathbf{1 4}} \mathbf{I}_{\mathbf{2}} \mathbf{N O} \mathbf{O}_{5} \mathbf{S}$ (458.9637): found: 458.9626. $\mathbf{C}_{\mathbf{1 6}} \mathbf{H}_{\mathbf{1 4}} \mathbf{I}_{\mathbf{2}} \mathbf{N O} \mathbf{O}_{5} \mathrm{~S}$ : required: C: 41.84; H: 3.07; N: 3.05; found: C: 42.12; H: 3.42; N: 3.04. 


\section{Synthesis of di(tert-butyl) 2-allyl-4-nitrophenylimidodicarbonate (27e)}

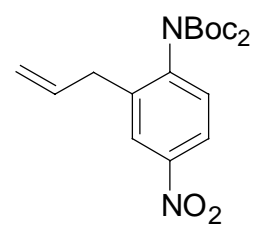

Prepared according to TP 5 form di(tert-butyl) 2-iodo-4-nitrophenylimidodicarbonate (1t) (446 mg, $1.00 \mathrm{mmol}), \mathrm{PhMgCl}(0.65 \mathrm{~mL}, 1.1 \mathrm{mmol}, 1.6 \mathrm{M}$ in THF), CuCN.2LiCl (1.1 mL, $1.1 \mathrm{mmol}, 1.0 \mathrm{M}$ in THF) and allyl bromide $(0.17 \mathrm{~mL}, 1.6 \mathrm{mmol})$. Purification by flash chromatography (pentane/diethyl ether $=9: 1$ ) furnished title compound $27 \mathbf{e}$ as a colourless oil (220 mg, 61\%).

${ }^{1} \mathbf{H}$-NMR $\left(300 \mathrm{MHz}, \mathrm{CDCl}_{3}, 25^{\circ} \mathrm{C}\right): \delta=8.14\left(\mathrm{~d},{ }^{4} J(\mathrm{H}, \mathrm{H})=2.7 \mathrm{~Hz}, 1 \mathrm{H}\right), 8.08(\mathrm{dd}$, $\left.{ }^{3} J(\mathrm{H}, \mathrm{H})=8.4 \mathrm{~Hz},{ }^{4} J(\mathrm{H}, \mathrm{H})=2.7 \mathrm{~Hz}, 1 \mathrm{H}\right), 7.25\left(\mathrm{~d},{ }^{3} J(\mathrm{H}, \mathrm{H})=8.4 \mathrm{~Hz}, 1 \mathrm{H}\right), 5.91-5.80(\mathrm{~m}, 1 \mathrm{H})$, 5.22-5.14 (m, $2 \mathrm{H}), 3.35\left(\mathrm{~d},{ }^{3} J(\mathrm{H}, \mathrm{H})=6.6 \mathrm{~Hz}, 2 \mathrm{H}\right), 1.39(\mathrm{~s}, 18 \mathrm{H}) .{ }^{13} \mathbf{C}-\mathbf{N M R}(75 \mathrm{MHz}$, $\left.\mathrm{CDCl}_{3}, 25^{\circ} \mathrm{C}\right): \delta=150.8,147.6,144.1,140.0,134.3,130.1,125.2,122.5,118.7,89.1,84.0$, 35.7, 28.3. MS (FAB, NBA): $m / z(\%): 401(32)[\mathrm{M}+\mathrm{Na}]^{+}, 379(8)[\mathrm{M}+\mathrm{H}]^{+} . \mathbf{M S}(70 \mathrm{eV}, \mathrm{EI})$, $m / z$ (\%): 278 (0.1) [M-Boc] $]^{+}, 269$ (1), 222 (11), 207 (8), 178 (7), 153 (14), 136 (20), 107 (21), 89 (30), 77 (63), 57 (100). IR (KBr): $\tilde{v}=2981$ (m), 1796 (vs), 1759 (s), 1723 (s), 1527 (vs), 1480 (m), 1394 (m), 1369 (vs), 1349 (vs), 1315 (s), 1276 (vs), 1251 (vs), 1154 (vs), 1116 (vs), 1099 (vs), 1006 (m), 869 (w), 851 (w), 832 (w), 812 (w), 778 (w). HRMS for $\mathbf{C}_{19} \mathbf{H}_{26} \mathbf{N}_{2} \mathbf{O}_{6}\left(401.1688[\mathrm{M}+\mathrm{Na}]^{+}\right)$: found: $401.1692[\mathrm{M}+\mathrm{Na}]^{+}$.

\section{Synthesis of $\{5$-nitro-2-[(E)-1-pyrrolidinyldiazenyl $]$ phenyl $\}$ (phenyl)methanol (27f)}

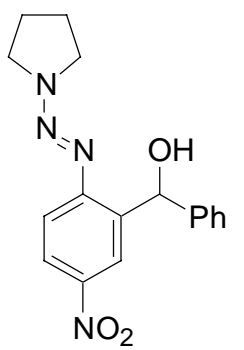

Prepared according to TP 5 from 1-[(E)-(2-iodo-4-nitrophenyl)diazenyl]pyrrolidine (1u) (346 mg, $1.00 \mathrm{mmol}), \mathrm{PhMgCl}(0.65 \mathrm{~mL}, 1.1 \mathrm{mmol}, 1.6 \mathrm{M}$ in THF), and benzaldehyde $(168 \mathrm{mg}, \quad 1.50 \mathrm{mmol})$ in a THF/NMP-mixture $3: 1$ at $-55^{\circ} \mathrm{C}$. Purification by flash chromatography (pentane/diethyl ether $=2: 1$ ) furnished title compound $\mathbf{2 7 f}$ as a yellow solid (159 mg, 58\%).

mp.: $171-171.5{ }^{\circ} \mathrm{C} .{ }^{1} \mathbf{H}-\mathbf{N M R}\left(300 \mathrm{MHz}, \mathrm{CDCl}_{3}, 25^{\circ} \mathrm{C}\right): \delta=8.22\left(\mathrm{~d},{ }^{4} J(\mathrm{H}, \mathrm{H})=2.7 \mathrm{~Hz}\right.$, $1 \mathrm{H}), 7.94\left(\mathrm{dd},{ }^{3} J(\mathrm{H}, \mathrm{H})=8.9 \mathrm{~Hz},{ }^{4} J(\mathrm{H}, \mathrm{H})=2.7 \mathrm{~Hz}, 1 \mathrm{H}\right), 7.46\left(\mathrm{~d},{ }^{4} J(\mathrm{H}, \mathrm{H})=8.9 \mathrm{~Hz}, 1 \mathrm{H}\right), 7.29$ $7.08(\mathrm{~m}, 5 \mathrm{H}), 6.31(\mathrm{~s}, 1 \mathrm{H}), 3.86\left(\mathrm{br} \_\mathrm{t},{ }^{3} J(\mathrm{H}, \mathrm{H})=6.6 \mathrm{~Hz}, 2 \mathrm{H}\right), 3.49\left(\mathrm{br} \_\mathrm{t},{ }^{3} J(\mathrm{H}, \mathrm{H})=6.6 \mathrm{~Hz}\right.$, $2 \mathrm{H}), 2.00-1.91(\mathrm{~m}, 4 \mathrm{H}) .{ }^{13} \mathrm{C}-\mathrm{NMR}\left(75 \mathrm{MHz}, \mathrm{CDCl}_{3}, 25^{\circ} \mathrm{C}\right): \delta=152.4,144.0,143.4,137.8$, 
127.9, 126.8, 126.4, 123.1, 123.0, 116.1, 72.5, 51.4, 47.1, 23.5, 23.1. MS (70 eV, EI), $\mathrm{m} / \mathrm{z}$ (\%): 326 (1) [ $\left.\mathrm{M}^{+}\right], 308$ (8), 256 (7), 242 (41), 210 (56), 181 (544), 164 (44), 152 (30), 105 (8), 85 (100), 79 (12). IR (KBr): $\widetilde{v}=3400$ (m), 1599 (w), 1581 (m), 1506 (vs), 1396 (vs), 1326 (vs), 1300 (vs), 1289 (vs), 1267 (vs), 1233 (s), 1084 (m), 1036 (m), 913 (w), 843 (m), 739 (w), 702 (w). $\mathbf{H R M S}$ for $\mathbf{C}_{\mathbf{1 7}} \mathbf{H}_{\mathbf{1 8}} \mathbf{N}_{\mathbf{4}} \mathbf{O}_{\mathbf{3}}$ (326.1379): found: 326.1397.

\section{Synthesis of 1-[(E)-(2-allyl-4-nitrophenyl)diazenyl]pyrrolidine (27g)}

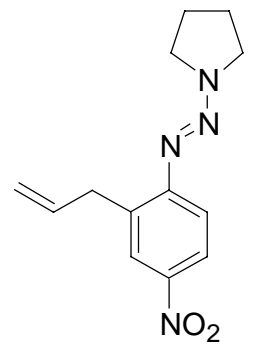

Prepared according to TP 5 from 1-[(E)-(2-allyl-4-nitrophenyl)diazenyl]pyrrolidine (1u) (346 mg, $1.00 \mathrm{mmol}), \mathrm{PhMgCl}(0.65 \mathrm{~mL}, 1.1 \mathrm{mmol}, 1.6 \mathrm{M}$ in THF), CuCN.2LiCl (1.1 mL, $1.1 \mathrm{mmol}, 1.0 \mathrm{M}$ in THF) and allyl bromide $(0.17 \mathrm{~mL}, 1.6 \mathrm{mmol})$ in a THF/NMP-mixture $3: 1$ at $-55^{\circ} \mathrm{C}$. Purification by flash chromatography (pentane/diethyl ether $=4: 1$ ) furnished title compound $27 \mathrm{~g}$ as a yellow solid $(159 \mathrm{mg}, 61 \%)$.

mp.: $\quad 88.5-89{ }^{\circ} \mathrm{C} .{ }^{1} \mathbf{H}-\mathrm{NMR}\left(300 \mathrm{MHz}, \mathrm{CDCl}_{3}, 25^{\circ} \mathrm{C}\right): \delta=8.06\left(\mathrm{~d},{ }^{4} J(\mathrm{H}, \mathrm{H})=2.7 \mathrm{~Hz}, 1 \mathrm{H}\right)$, $8.00\left(\mathrm{dd},{ }^{3} J(\mathrm{H}, \mathrm{H})=8.9 \mathrm{~Hz},{ }^{4} J(\mathrm{H}, \mathrm{H})=2.7 \mathrm{~Hz}, 1 \mathrm{H}\right), 7.49\left(\mathrm{~d},{ }^{4} J(\mathrm{H}, \mathrm{H})=8.9 \mathrm{~Hz}, 1 \mathrm{H}\right), 6.06-5.93$ $(\mathrm{m}, 1 \mathrm{H}), 5.13-5.04(\mathrm{~m}, 2 \mathrm{H}), 3.98\left(\mathrm{br} \_\mathrm{t}, 2 \mathrm{H}\right), 3.69\left(\mathrm{br} \_\mathrm{t}, 2 \mathrm{H}\right), 3.63\left(\mathrm{~d},{ }^{3} J(\mathrm{H}, \mathrm{H})=6.6 \mathrm{~Hz}\right.$, $2 \mathrm{H}), 2.10-2.03$ (m, $4 \mathrm{H}) .{ }^{13} \mathrm{C}-\mathbf{N M R}\left(75 \mathrm{MHz}, \mathrm{CDCl}_{3}, 25{ }^{\circ} \mathrm{C}\right): \delta=153.7,144.4,136.4,135.1$, 125.3, 122.6, 116.3, 116.2, 51.4, 47.0, 35.5, 23.9, 23.4. MS (70 eV, EI), m/z (\%): 259 (4) [M $\left.\mathrm{M}^{+}\right], 231$ (3), 217 (3), 190 (18), 145 (12), 131 (7), 115 (100),104 (7), 89 (7), 70 (7). IR (KBr): $\tilde{v}=2975(\mathrm{w}), 1636(\mathrm{w}), 1582(\mathrm{~m}), 1503$ (vs), 1455 (m), 1389 (vs), 1325 (vs), 1305 (vs), 1268 (vs), 1231 (s), 1101 (m), 915 (m), 898 (m), 832 (w), 757 (w). HRMS for $\mathbf{C}_{\mathbf{1 3}} \mathbf{H}_{16} \mathbf{N}_{\mathbf{4}} \mathbf{O}_{\mathbf{2}}$ (260.1273): found: 260.1297 .

\section{Synthesis of \{5-nitro-2-[1-pyrrolidinyldiazenyl]phenyl\}(phenyl)methanone (27h)}

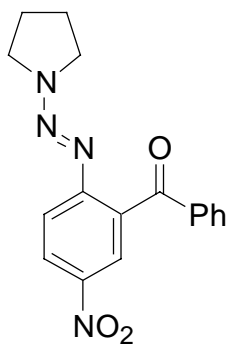

Prepared according to TP 5 from 1-[(E)-(2-allyl-4-nitrophenyl)diazenyl]pyrrolidine (1u) (346 mg, $1.00 \mathrm{mmol}), \mathrm{PhMgCl}(0.65 \mathrm{~mL}, 1.1 \mathrm{mmol}, 1.6 \mathrm{M}$ in THF), CuCN.2LiCl (1.1 mL, 
$1.1 \mathrm{mmol}, 1.0 \mathrm{M}$ in THF) and benzoyl bromide (370 $\mathrm{mg}, 2.00 \mathrm{mmol})$ in a THF/NMP-mixture $3: 1$ at $-55^{\circ} \mathrm{C}$. Purification by flash chromatography (pentane/diethyl ether $=3: 1$ ) furnished title compound $\mathbf{2 7 h}$ as a yellow solid $(159 \mathrm{mg}, 75 \%)$.

mp.: $\quad 172.5-173{ }^{\circ} \mathrm{C} .{ }^{\mathbf{1}} \mathbf{H}-\mathbf{N M R}\left(400 \mathrm{MHz}, \mathrm{CDCl}_{3}, 25^{\circ} \mathrm{C}\right): \delta=8.30-8.26(\mathrm{~m}, 2 \mathrm{H}), 7.73-7.69$ $(\mathrm{m}, 2 \mathrm{H}), 7.62\left(\mathrm{~d},{ }^{3} J(\mathrm{H}, \mathrm{H})=8.9 \mathrm{~Hz}, 1 \mathrm{H}\right), 7.51\left(\mathrm{tt},{ }^{3} J(\mathrm{H}, \mathrm{H})=7.5 \mathrm{~Hz},{ }^{4} J(\mathrm{H}, \mathrm{H})=2.0 \mathrm{~Hz}, 1 \mathrm{H}\right)$, 7.40-7.36 (m, $2 \mathrm{H}), 3.74$ (br_t, $2 \mathrm{H}), 3.09$ (br_t, $2 \mathrm{H}), 1.87-1.81$ (m, $4 \mathrm{H}) .{ }^{\mathbf{1 3}} \mathbf{C}-\mathbf{N M R}$ $\left(100 \mathrm{MHz}, \mathrm{CDCl}_{3}, 25^{\circ} \mathrm{C}\right): \delta=195.9,154.3,144.2,137.7,133.5,132.7,129.3,128.3,125.9$,

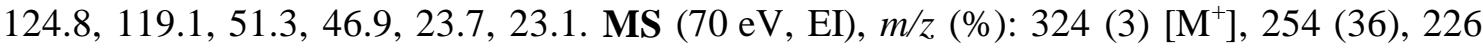
(12), 195 (7), 180 (100), 152 (37), 139 (6), 105 (41), 77 (40). IR (KBr): $\tilde{v}=1667$ (vs), 1597 (m), 1578 (w), 1511 (s), 1398 (vs), 1333 (vs), 1306 (vs), 1270 (vs), 1147 (m), 970 (m), 862 (w), 713 (m). HRMS for $\mathbf{C}_{\mathbf{1 7}} \mathbf{H}_{\mathbf{1 6}} \mathbf{N}_{\mathbf{4}} \mathbf{O}_{\mathbf{3}}$ (324.1222): found: 324.1226.

\section{Synthesis of 1-allyl-5-nitrobenzophenone (27i)}

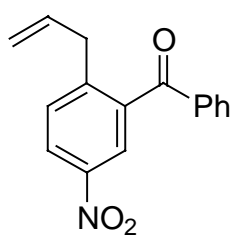

Prepared according to TP 5 from 3,5-diiodo-2,6-dimethyl-nitrobenzene (1n) $(352 \mathrm{mg}$, $1.00 \mathrm{mmol}), \mathrm{PhMgCl}(0.65 \mathrm{~mL}, 1.1 \mathrm{mmol}, 1.6 \mathrm{M}$ in THF), CuCN.2LiCl (1.1 mL, $1.1 \mathrm{mmol}$, $1.0 \mathrm{M}$ in THF) and allyl bromide $(0.17 \mathrm{~mL}, 1.50 \mathrm{mmol})$ at $-78{ }^{\circ} \mathrm{C}$. Reaction time $1 \mathrm{~h}$. Purification by flash chromatography (pentane/diethyl ether $=19: 1$ ) furnished title compound $\mathbf{2 7 i}$ as a yellow oil (144 $\mathrm{mg}, 54 \%)$.

${ }^{1}$ H-NMR $\left(300 \mathrm{MHz}, \mathrm{CDCl}_{3}, 25{ }^{\circ} \mathrm{C}\right): \delta=8.25\left(\mathrm{dd},{ }^{3} J(\mathrm{H}, \mathrm{H})=8.4 \mathrm{~Hz},{ }^{4} J(\mathrm{H}, \mathrm{H})=2.2 \mathrm{~Hz}, 1 \mathrm{H}\right)$, $8.15\left(\mathrm{~d},{ }^{4} J(\mathrm{H}, \mathrm{H})=2.2 \mathrm{~Hz}, \quad 1 \mathrm{H}\right), \quad 7.79-7.75 \quad(\mathrm{~m}, \quad 2 \mathrm{H}), \quad 7.51 \quad\left(\mathrm{tt}, \quad{ }^{3} J(\mathrm{H}, \mathrm{H})=7.5 \mathrm{~Hz}\right.$, $\left.{ }^{4} J(\mathrm{H}, \mathrm{H})=1.4 \mathrm{~Hz}, 1 \mathrm{H}\right), 7.54-7.46(\mathrm{~m}, 3 \mathrm{H}), 5.91-5.77(\mathrm{~m}, 1 \mathrm{H}), 5.07-4.96(\mathrm{~m}, 2 \mathrm{H}), 3.53-3.50$ (m, $2 \mathrm{H}) .{ }^{13} \mathbf{C}-\mathbf{N M R}\left(75 \mathrm{MHz}, \mathrm{CDCl}_{3}, 25{ }^{\circ} \mathrm{C}\right): \delta=196.1,146.9,146.3,140.1,136.9,135.3$, 134.5, 131.8, 130.5, 129.2, 125.2, 123.7, 118.3, 37.7. MS (70 eV, EI), m/z (\%): 267 (30) $\left[\mathrm{M}^{+}\right]$, 252 (100), 237 (15), 220 (18), 206 (29), 202 (16), 189 (19), 165 (32), 115 (53), 105 (56), 77 (90), 51 (21). IR (KBr): $\widetilde{v}=1669$ (vs), 1610 (w), 1596 (w), 1581 (w), 1525 (vs), 1449 (m), 1350 (vs), 1316 (m), 1261 (s), 1075 (w), 999 (w), 962 (w), 920 (m), 869 (m), 796 (m), 740 (m), 703 (m), 642 (m). HRMS for $\mathbf{C}_{\mathbf{1 6}} \mathbf{H}_{\mathbf{1 3}} \mathbf{N O}_{\mathbf{3}}$ (267.0895): found: 267.0899 .

\section{Synthesis of (5-iodo-2,4-dimethyl-3-nitrophenyl)(phenyl)methanol (27j)}<smiles>Cc1c(I)cc(C(O)c2ccccc2)c([N+](=O)[O-])c1[N+](=O)[O-]</smiles> 
Prepared according to TP 5 from 3,5-diiodo-2,6-dimethyl-nitrobenzene (1s) (402 mg, $1.00 \mathrm{mmol}), \mathrm{PhMgCl}(0.65 \mathrm{~mL}, 1.1 \mathrm{mmol}, 1.6 \mathrm{M}$ in THF), and benzaldehyde (168 mg, $1.50 \mathrm{mmol}$ ) at $-40^{\circ} \mathrm{C}$. Exchange was finished after $30 \mathrm{~min}$. Reaction time $1 \mathrm{~h}$. Purification by flash chromatography (pentane/diethyl ether $=19: 1$ ) furnished title compound $\mathbf{2 7} \mathbf{j}$ as a yellow oil (159 mg, 58\%).

${ }^{1}$ H-NMR $\left(300 \mathrm{MHz}, \mathrm{CDCl}_{3}, 25{ }^{\circ} \mathrm{C}\right): \delta=8.15(\mathrm{~s}, 1 \mathrm{H}), 7.30-7.13(\mathrm{~m}, 5 \mathrm{H}), 5.75(\mathrm{~s}, 1 \mathrm{H}), 2.46$ $(\mathrm{s}, 1 \mathrm{OH}), 2.27(\mathrm{~s}, 3 \mathrm{H}), 1.88(\mathrm{~s}, 3 \mathrm{H}) .{ }^{13} \mathrm{C}-\mathrm{NMR}\left(75 \mathrm{MHz}, \mathrm{CDCl}_{3}, 25{ }^{\circ} \mathrm{C}\right): \delta=152.6,142.2$, $141.1,138.0,131.1,128.9,128.4,127.1,126.4,98.7,72.7,23.2,13.6 . \mathrm{MS}(70 \mathrm{eV}, \mathrm{EI}), \mathrm{m} / \mathrm{z}$ (\%): 383 (81) [ $\left.\mathrm{M}^{+}\right], 348$ (62), 303 (44), 288 (12), 221 (12), 193 (36), 165 (30), 127 (18), 105 (100), 77 (56). IR (KBr): $\tilde{v}=3350$ (m), 1531 (vs), 1454 (s), 1382 (s), 1359 (vs), 1164 (m), 1059 (m), 1016 (m), 891 (w), 767 (m), 742 (s), 700 (s). HRMS for $\mathbf{C}_{\mathbf{1 5}} \mathbf{H}_{\mathbf{1 4}} \mathbf{I N O}_{\mathbf{3}}$ (383.0018): found: 383.0018 .

\section{Synthesis of compounds 29}

\section{Synthesis of ethyl 4-nitro[1,1'-biphenyl]-2-carboxylate (29a)}

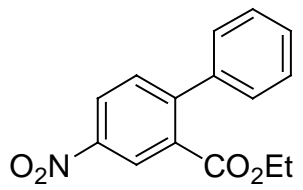

Prepared according to TP 6 from ethyl 2-iodo-5-nitrobenzoate (1i) (321 mg, $1.00 \mathrm{mmol}$ ), $\mathrm{PhMgCl}\left(0.65 \mathrm{~mL}, 1.1 \mathrm{mmol}, 1.7 \mathrm{M}\right.$ in THF), $\mathrm{ZnBr}_{2}(1.1 \mathrm{~mL}, 1.1 \mathrm{mmol}, 1.0 \mathrm{M}$ in THF), $\mathrm{Pd}(\mathrm{dba})_{2}(29 \mathrm{mg}, 0.05 \mathrm{mmol})$ and $\mathrm{tfp}(23 \mathrm{mg}, 0.10 \mathrm{mmol})$. Reaction time: $4 \mathrm{~h}$ at $20^{\circ} \mathrm{C}$. Purification by flash chromatography (pentane/diethyl ether $=19: 1$ ) furnished biphenyl 29a as a colourless solid (184 mg, 68\%).

mp.: $\quad 64.8-66.0{ }^{\circ} \mathrm{C} .{ }^{1} \mathbf{H}-\mathrm{NMR}\left(300 \mathrm{MHz}, \mathrm{CDCl}_{3}, 25^{\circ} \mathrm{C}\right): \delta=8.60(\mathrm{~d}, 1 \mathrm{H}), 8.27(\mathrm{dd}$, $\left.{ }^{4} J(\mathrm{H}, \mathrm{H})=2.7 \mathrm{~Hz},{ }^{3} J(\mathrm{H}, \mathrm{H})=8.7 \mathrm{~Hz}, 1 \mathrm{H}\right), 7.48(\mathrm{~d}, 1 \mathrm{H}), 7.37-7.36(\mathrm{~m}, 2 \mathrm{H}), 7.35-7.34(\mathrm{~m}$, $2 \mathrm{H}), 7.26-7.23(\mathrm{~m}, 1 \mathrm{H}), 4.07\left(\mathrm{q},{ }^{3} J(\mathrm{H}, \mathrm{H})=7.1 \mathrm{~Hz}, 2 \mathrm{H}\right), 0.97\left(\mathrm{t},{ }^{3} J(\mathrm{H}, \mathrm{H})=7.1 \mathrm{~Hz}, 3 \mathrm{H}\right)$. ${ }^{13}$ C-NMR $\left(75 \mathrm{MHz}, \mathrm{CDCl}_{3}, 25{ }^{\circ} \mathrm{C}\right): \delta=165.6,147.6,145.8,138.3,131.6,130.8,127.4$, 127.3, 127.1, 124.5, 124.0, 60.8, 12.6. MS (70 eV, EI), $\mathrm{m} / z(\%): 271(61)\left[\mathrm{M}^{+}\right], 243$ (18), 226 (100), 210 (3), 196 (2), 180 (39), 168 (2), 152 (37), 139 (12), 126 (4), 115 (3). IR (KBr): $\widetilde{v}=1720(\mathrm{vs}), 1609(\mathrm{w}), 1575$ (w), 1524 (s), 1349 (s), 1283 (s), $1236(\mathrm{w}), 1143$ (m), 1115 (w), 777 (m), 759 (w), 742 (m), 699 (m). $\mathbf{C}_{\mathbf{1 5}} \mathbf{H}_{\mathbf{1 3}} \mathbf{N O}_{\mathbf{4}}$ : required: C: 66.41; H: 4.83; N: 5.16; found: C: 66.57; H: 4.89; N: 5.24. 


\section{Synthesis of ethyl 4'-cyano-4-nitro[1,1'-biphenyl]-2-carboxylate (29b)}<smiles>CCOC(=O)c1cc([N+](=O)[O-])ccc1-c1ccc(C#N)cc1</smiles>

Prepared according to TP 6 from ethyl 2-iodo-5-nitrobenzoate (1i) (321 mg, $1.00 \mathrm{mmol}$ ), $\mathrm{PhMgCl}(0.65 \mathrm{~mL}, 1.1 \mathrm{mmol}, 1.7 \mathrm{M}$ in $\mathrm{THF}), \mathrm{ZnBr}_{2}(1.1 \mathrm{~mL}, 1.1 \mathrm{mmol}, 1.0 \mathrm{M}$ in $\mathrm{THF})$, $\mathrm{Pd}(\mathrm{dba})_{2}(29 \mathrm{mg}, 0.05 \mathrm{mmol}), \mathrm{tfp}(23 \mathrm{mg}, 0.10 \mathrm{mmol})$ and 4-iodo-benzonitrile (21h) $(458 \mathrm{mg}$, $2.00 \mathrm{mmol})$. Reaction time: $2 \mathrm{~h}$ at $20^{\circ} \mathrm{C}$. Purification by flash chromatography (pentane/diethyl ether $=2: 1$ ) yielded biphenyl $\mathbf{2 9 b}$ as a pale yellow solid $(245 \mathrm{mg}, 83 \%$ ).

mp.: $\quad 105-106{ }^{\circ} \mathrm{C} .{ }^{1} \mathbf{H}-\mathbf{N M R}\left(300 \mathrm{MHz}, \mathrm{CDCl}_{3}, 25^{\circ} \mathrm{C}\right): \delta=8.77\left(\mathrm{~d},{ }^{4} J(\mathrm{H}, \mathrm{H})=2.8 \mathrm{~Hz}, 1 \mathrm{H}\right)$, $8.40\left(\mathrm{dd},{ }^{3} J(\mathrm{H}, \mathrm{H})=8.4 \mathrm{~Hz},{ }^{4} J(\mathrm{H}, \mathrm{H})=2.8 \mathrm{~Hz}, 1 \mathrm{H}\right), 7.72\left(\mathrm{~d},{ }^{3} J(\mathrm{H}, \mathrm{H})=8.8 \mathrm{~Hz}, 2 \mathrm{H}\right), 7.53(\mathrm{~d}$, $\left.{ }^{3} J(\mathrm{H}, \mathrm{H})=8.4 \mathrm{~Hz}, 1 \mathrm{H}\right), 7.40\left(\mathrm{~d},{ }^{3} J(\mathrm{H}, \mathrm{H})=8.8 \mathrm{~Hz}, 2 \mathrm{H}\right), 4.20\left(\mathrm{q},{ }^{3} J(\mathrm{H}, \mathrm{H})=7.1 \mathrm{~Hz}, 2 \mathrm{H}\right), 1.12$ $\left(\mathrm{d},{ }^{3} J(\mathrm{H}, \mathrm{H})=7.1 \mathrm{~Hz}, 3 \mathrm{H}\right) .{ }^{13} \mathbf{C}-\mathbf{N M R}\left(75 \mathrm{MHz}, \mathrm{CDCl}_{3}, 25{ }^{\circ} \mathrm{C}\right): \delta=165.3,147.4,146.8$, 144.1, 132.0, 131.9, 131.8, 128.9, 126.0, 125.5, 118.3, 112.2, 62.0, 13.7. MS (70 eV, EI), $\mathrm{m} / \mathrm{z}$ (\%): 296 (51) $\left[\mathrm{M}^{+}\right], 268$ (53), 251 (100), 205 (32), 177 (28), 150 (12), 140 (8). IR (KBr): $\tilde{v}=2228$ (s), 1710 (vs), 1609 (s), 1525 (vs), 1474 (s), 1419 (m), 1353 (vs), 1304 (s), 1283 (vs), 1262 (m), 1153 (s), 1143 (s), 1006 (s), 837 (m), 733 (m). HRMS for $\mathbf{C}_{\mathbf{1 6}} \mathbf{H}_{\mathbf{1 2}} \mathbf{N}_{\mathbf{2}} \mathbf{O}_{\mathbf{4}}$ (296.0797): found: 296.0865. $\mathbf{C}_{\mathbf{1 6}} \mathbf{H}_{\mathbf{1 2}} \mathbf{N}_{\mathbf{2}} \mathbf{O}_{\mathbf{4}}$ : required: C: 64.86; H: 4.08; N: 9.20; found: C: 65.14; H: 4.09; N: 9.20.

\footnotetext{
${ }^{1}$ Sy, W.-W. Synth. Comm. 1992, 22, 3215.

${ }^{2}$ Searle, N. E.; Adams, R. J. Am. Chem. Soc. 1933, 55, 1649.

${ }^{3}$ Baik, W. P.; Kim, J. M.; Kim, Y. S.; Yoon, C. H.; Kim, S. J.; Lee, S. W. PCT Int. Appl. 2002 WO 2001-KR1586.

${ }^{4}$ Grehn, L.; Gunnarsson, K.; Rugnarsson, U. Acta Chem. Scand. 1986, B40, 745.
} 